Supporting information I

\title{
Direct Synthesis of Polyaryls by Consecutive Oxidative Cross-Coupling of Phenols with Arenes
}

Alina Dyadyuk, ${ }^{\dagger}$ Kavitha Sudheendran,${ }^{\dagger}$ Yulia Vainer,${ }^{\dagger}$ Vlada Vershinin,${ }^{\dagger}$ Alexander I. Shames, ${ }^{\ddagger}$ Doron Pappo*, ${ }^{* \dagger}$

'Department of Chemistry, Ben-Gurion University of the Negev, Beer-Sheva 84105, Israel

tDepartment of Physics, Ben-Gurion University of the Negev, Beer-Sheva 84105, Israel

Email: pappod@bgu.ac.il

Table of Contents

1. General information. . $\mathrm{S} 2$

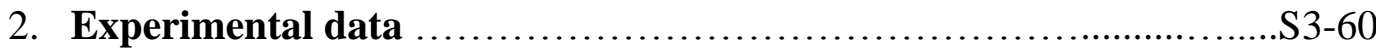

2.1 Synthesis of starting biaryls.............................................. $3-6$

2.1.1 General Suzuki coupling procedure.............................S3

2.1.2 Characterization data...................................... $3-6$

2.2 Sequential cross-coupling products..............................S6-21

2.2.1 General Sequential cross-coupling procedure.......................S6

2.2.2 Characterization data for compounds..........................S7-21

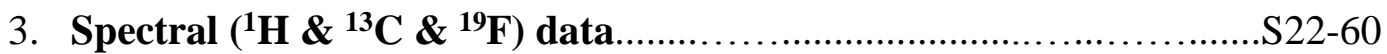




\section{General Information:}

All reagents were of reagent grade quality, purchased commercially from SigmaAldrich, Alfa-Aesar, or Fluka, and used without further purification. $\mathrm{FeCl}_{3}$ anhydrous 98\% purchased from Strem Chemicals. Purification by column chromatography was performed on Fluka chromatographic silica gel $(40-63 \mu \mathrm{m})$. TLC analyses were performed using Merck silica gel glass plates 60 F254. NMR spectra were recorded on Bruker DPX400, or DMX500 instruments; chemical shifts are given in ppm and are relative to $\mathrm{Me}_{4} \mathrm{Si}$ as an internal standard or to the residual solvent peak. IR spectra were recorded on NICOLET 380 FT-IR instrument. HR-MS data were obtained using a Thermoscientific LTQU XL Orbitrap HRMS equipped with APCI (atmosphericpressure chemical ionization). HPLC analysis was carried out on Agilent 1260 instrument equipped with a G4212-60008 photodiode array detector, ES-MS Advion Expression unit and an Agilent reverse phase ZORBAX Eclipse plus C18 $3.5 \mu \mathrm{m}$ column (4.6 X $100 \mathrm{~mm})$. 


\section{Experimental data:}

\subsection{Synthesis of starting biaryls:}

\subsubsection{General Suzuki coupling procedure:}

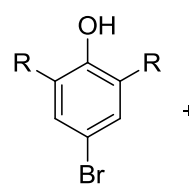

$\mathrm{R}=\mathrm{Me}, \mathrm{OMe}$<smiles>OB(O)c1cc[R]cc1</smiles>

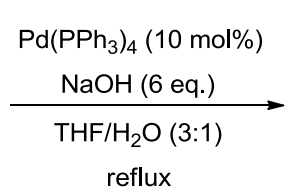<smiles>[R]c1cc(-c2ccccc2)cc([R])c1O</smiles>

2.1.2 General procedure: A mixture of 4-bromo-2,6-dimethyl phenol (200 mg, 1 mmol), arylboronic acid (1.5 mmol), $\mathrm{Pd}\left(\mathrm{PPh}_{3}\right)_{4}(115 \mathrm{mg}, 10 \mathrm{~mol} \%)$ and $\mathrm{NaOH}(240$ $\mathrm{mg}, 6 \mathrm{mmol})$ in $\mathrm{THF} / \mathrm{H}_{2} \mathrm{O}(3: 1,18 \mathrm{~mL})$ were deoxygenated under vacuum and refilled with nitrogen for 3 times. The reaction was stirred at $75^{\circ} \mathrm{C}$ for $16 \mathrm{~h}$, cooled and ethyl acetate $(15 \mathrm{~mL})$ and water $(10 \mathrm{~mL})$ were added. The organic phase was separated and the water phase was extracted with ethyl acetate $(2 \times 15 \mathrm{~mL})$. The combined organic phases were washed with brine, dried over $\mathrm{Na}_{2} \mathrm{SO}_{4}$ and evaporated under vacuum. The crude residue was purified by column chromatography.

\subsubsection{Characterization data:}<smiles>COc1cc(OC)c(-c2cc(C)c(O)c(OC)c2)c(OC)c1</smiles>

1b

Compound 1b: compound 1b was prepared from 4-bromo-2,6dimethyl phenol (200 $\mathrm{mg}, 1 \mathrm{mmol})$ and $(2,4,6-$ trimethoxyphenylboronic acid $(318 \mathrm{mg}, 1.5 \mathrm{mmol})$ according to the general procedure. The crude residue was purified by column chromatography (hexane/ethyl acetate 9:1) to afford compound $1 \mathrm{~b}$ (115 mg, 40\% yield) as a white solid.

Characterization data of compound 1b: ${ }^{1} \mathrm{H} \mathrm{NMR}\left(400 \mathrm{MHz}, \mathrm{CDCl}_{3}\right) \delta 6.95(\mathrm{~s}, 2 \mathrm{H})$, 6.23 (s, 2H), 4.62 (s, 1H), 3.87 (s, 3H), 3.74 (s, 6H), 2.27 (s, 6H); ${ }^{13} \mathrm{C}$ NMR (100 MHz, $\left.\mathrm{CDCl}_{3}\right) \delta 160.2,158.6,151.1,131.4,125.7,122.5,112.4,90.9,56.0,55.5,16.2 ; \mathrm{HRMS}$ (ESI): $m / z$ calcd for $\mathrm{C}_{17} \mathrm{H}_{21} \mathrm{O}_{4}[\mathrm{M}+\mathrm{H}]^{+} 289.1434$, found 289.1437 . 


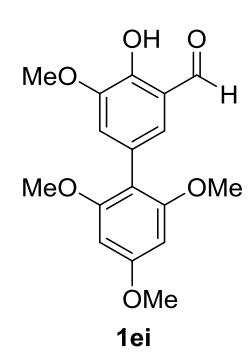

Compound 1ei: A mixture of 5-bromo-2-hydroxy-3methoxybenzaldehyde (347 mg, $1.5 \mathrm{mmol})$, (2,4,6-trimethoxy phenyl)boronic acid (477 mg, $2.25 \mathrm{mmol}), \mathrm{Pd}\left(\mathrm{PPh}_{3}\right)_{4}(173 \mathrm{mg}, 10 \mathrm{~mol}$ $\%$ ) and $\mathrm{NaOH}(360 \mathrm{mg}, 9 \mathrm{mmol})$ in $\mathrm{THF} / \mathrm{H}_{2} \mathrm{O}(3: 1,27 \mathrm{~mL})$ were deoxygenated under vacuum and refilled with nitrogen for 3 times. Then stirring was continued for 3 days at $85{ }^{\circ} \mathrm{C}$. After cooling, ethyl acetate $(40 \mathrm{~mL})$ and water $(40 \mathrm{~mL})$ were added. The organic phase was separated and the water phase was extracted with ethyl acetate $(3 \times 30 \mathrm{~mL})$. The combined organic phase were washed with brine, dried over $\mathrm{Na}_{2} \mathrm{SO}_{4}$ and evaporated under vacuum. The crude residue was purified by column chromatography; hexane/ethyl acetate $4: 1$ to afford compound 1ei (143 mg, 30\% yield) as a yellow solid.

Characterization data of compound 1ei: ${ }^{1} \mathrm{H}$ NMR $\left(400 \mathrm{MHz}, \mathrm{CDCl}_{3}\right) \delta 9.89(\mathrm{~s}, 1 \mathrm{H})$, $7.16(\mathrm{~s}, 1 \mathrm{H}), 7.13(\mathrm{~s}, 1 \mathrm{H}), 6.24(\mathrm{~s}, 2 \mathrm{H}), 3.90(\mathrm{~s}, 3 \mathrm{H}), 3.87(\mathrm{~s}, 3 \mathrm{H}), 3.75(\mathrm{~s}, 6 \mathrm{H}) ;{ }^{13} \mathrm{C}$ NMR $\left(100 \mathrm{MHz}, \mathrm{CDCl}_{3}\right) \delta 197.0,160.9,158.4,150.2,147.5,127.4,125.7,122.0$, 120.5, 110.6, 90.9, 56.4, 55.9, 55.5; HRMS (ESI): $\mathrm{m} / z$ calcd for $\mathrm{C}_{17} \mathrm{H}_{19} \mathrm{O}_{6}[\mathrm{M}+\mathrm{H}]^{+}$ 319.1131, found 319.1176. IR (KBr pellet): 3420(m, broad), 1650(s), 1580 (s), 1120 (s) $\mathrm{cm}^{-1}$.

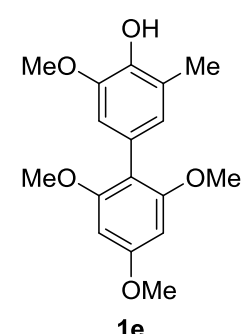

$1 \mathrm{e}$

Compound 1e: A mixture of compound 1ei (112 mg, $0.4 \mathrm{mmol})$ and $10 \% \mathrm{Pd} / \mathrm{C}(24 \mathrm{mg}, 40 \mathrm{~mol} \%)$ in methanol $(25 \mathrm{~mL})$ was stirred under a hydrogen atmosphere at room temperature for 5 days. Then the reaction mixture was filtered through short pad of Celite and the filtrate was evaporated. The crude residue was purified by column chromatography; hexane/ethyl acetate 1:1 to afford compound 1e (40 mg, $40 \%$ yield) as a white solid.

Characterization data of compound 1e: ${ }^{1} \mathrm{H}$ NMR $\left(400 \mathrm{MHz}, \mathrm{CDCl}_{3}\right) \delta 6.73(\mathrm{~s}, 1 \mathrm{H}), 6.72$ (s, 1H), 6.25 (s, 2H), 5.70 (s, 1H), 3.88 (s, 3H), 3.87 (s, 3H), 3.75 (s, 6H), 2.29 (s, 3H). ${ }^{13} \mathrm{C} \mathrm{NMR}\left(100 \mathrm{MHz}, \mathrm{CDCl}_{3}\right) \delta 160.3,158.6,145.7,142.6,123.00,124.8,112.7,111.5$, 91.0, $56.0(\times 2), 55.5,15.7$; HRMS (ESI): $\mathrm{m} / z$ calcd for $\mathrm{C}_{17} \mathrm{H}_{21} \mathrm{O}_{5}[\mathrm{M}+\mathrm{H}]^{+} 305.1384$, found 305.1377 . 


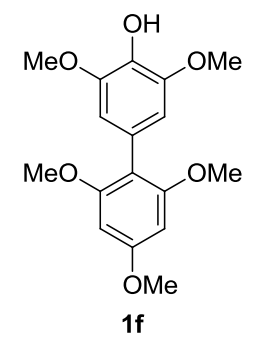

Compound 1f: A mixture of 4-bromo-2,6-dimethoxy-phenol (247 mg, $1 \mathrm{mmol}),(2,4,6$-trimethoxy)phenylboronic acid (318 $\mathrm{mg}, 1.5 \mathrm{mmol})$, $\mathrm{Pd}\left(\mathrm{PPh}_{3}\right)_{4}(115 \mathrm{mg}, 10 \mathrm{~mol} \%)$ and $\mathrm{NaOH}(240 \mathrm{mg}, 6 \mathrm{mmol})$ in $\mathrm{THF} / \mathrm{H}_{2} \mathrm{O}(3: 1,18 \mathrm{~mL})$ were deoxygenated under vacuum and refilled with nitrogen for 3 times. Then stirring was continued for $21 \mathrm{~h}$ at $85^{\circ} \mathrm{C}$. After cooling, ethyl acetate $(25 \mathrm{~mL})$ and water $(20 \mathrm{~mL})$ were added. The organic phase was separated and the water phase was extracted with ethyl acetate $(3 \times 30 \mathrm{~mL})$. The combined organic phase were washed with brine, dried over $\mathrm{Na}_{2} \mathrm{SO}_{4}$ and evaporated under vacuum. The crude residue was purified by column chromatography (hexane/ethyl acetate 7:3) to afford compound $\mathbf{1 f}(63 \mathrm{mg}, 20 \%$ yield) as a white solid. Characterization data of compound 1f: ${ }^{1} \mathrm{H}$ NMR $\left(400 \mathrm{MHz}, \mathrm{CDCl}_{3}\right) \delta 6.57$ (s, 2H), 6.24 (s, 2H), 5.52 (s, 1H), 3.87 (overlapped, 9H), $3.74(\mathrm{~s}, 6 \mathrm{H}) ;{ }^{13} \mathrm{C} \mathrm{NMR}\left(100 \mathrm{MHz}, \mathrm{CDCl}_{3}\right)$ $\delta 160.5,158.6,146.6,133.6,124.9,112.6,108.1,91.1,56.3,56.1,55.5$; HRMS (ESI): $m / z$ calcd for $\mathrm{C}_{17} \mathrm{H}_{19} \mathrm{O}_{6}[\mathrm{M}+\mathrm{H}]^{+} 319.1131$, found 319.1176 .

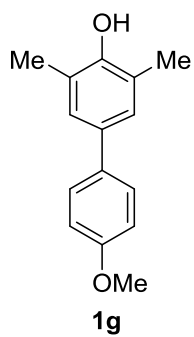

Compound 1g: Compound 1g was prepared from 4-bromo-2,6dimethylphenol (200 mg, $1 \mathrm{mmol}$ ) and 4-methoxyphenylboronic acid (228 $\mathrm{mg}, 1.5 \mathrm{mmol}$ ) according to the general conditions. The crude residue was purified by column chromatography (hexane/ethyl acetate 9:1) to afford compound $\mathbf{1 g}$ (157 $\mathrm{mg}, 69 \%$ yield) as a white solid.

Characterization data of compound 1g: ${ }^{1} \mathrm{H} \mathrm{NMR}\left(400 \mathrm{MHz}, \mathrm{CDCl}_{3}\right) \delta 7.47$ (dd, $J=8.4$ $\mathrm{Hz}, 2 \mathrm{H}), 7.18(\mathrm{~s}, 2 \mathrm{H}), 6.95(\mathrm{dd}, J=8.4 \mathrm{~Hz}, J=2.1 \mathrm{~Hz}, 2 \mathrm{H}), 3.85(\mathrm{~s}, 3 \mathrm{H}), 2.31(\mathrm{~s}, 6 \mathrm{H})$; ${ }^{13} \mathrm{C}$ NMR $\left(100 \mathrm{MHz}, \mathrm{CDCl}_{3}\right) \delta 158.7,151.5,133.9,133.2,128.7,127.9,127.1,123.4$, 114.2, 55.5, 16.2; HRMS (ESI): $\mathrm{m} / z$, calcd for $\mathrm{C}_{15} \mathrm{H}_{17} \mathrm{O}_{2}[\mathrm{M}+\mathrm{H}]^{+} 229.1223$, found 229.1228 . 


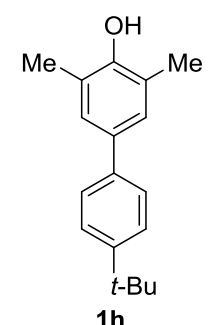

Compound 1h: Compound 1h was prepared from 4-bromo-2,6dimethylphenol (200 mg, $1 \mathrm{mmol})$ and 4-tert-butylphenylboronic acid ( $267 \mathrm{mg}, 1.5 \mathrm{mmol}$ ) according to the general procedure. The crude residue was purified by column chromatography (hexane/ethyl acetate 9:1) to afford compound $\mathbf{1 h}$ (182 $\mathrm{mg}, 72 \%$ yield) as a white solid.

Characterization data of compound $\mathbf{1 h}:{ }^{1} \mathrm{H}$ NMR $\left(500 \mathrm{MHz}, \mathrm{CDCl}_{3}\right) \delta 7.47(\mathrm{dd}, J=8.3$ $\mathrm{Hz}, 4 \mathrm{H}), 7.24$ (s, 2H), 4.66 (s, 1H), 2.33 (s, 6H), 1.38 (s, 9H); ${ }^{13} \mathrm{C}$ NMR (125 MHz, $\left.\mathrm{CDCl}_{3}\right) \delta 151.8,149.6,138.4,133.4,127.4,126.5,125.7,123.3,34.6,31.5,16.2$; HRMS (ESI): $m / z$ calcd for $\mathrm{C}_{18} \mathrm{H}_{23} \mathrm{O}[\mathrm{M}+\mathrm{H}]^{+} 255.1743$, found 255.1753 .

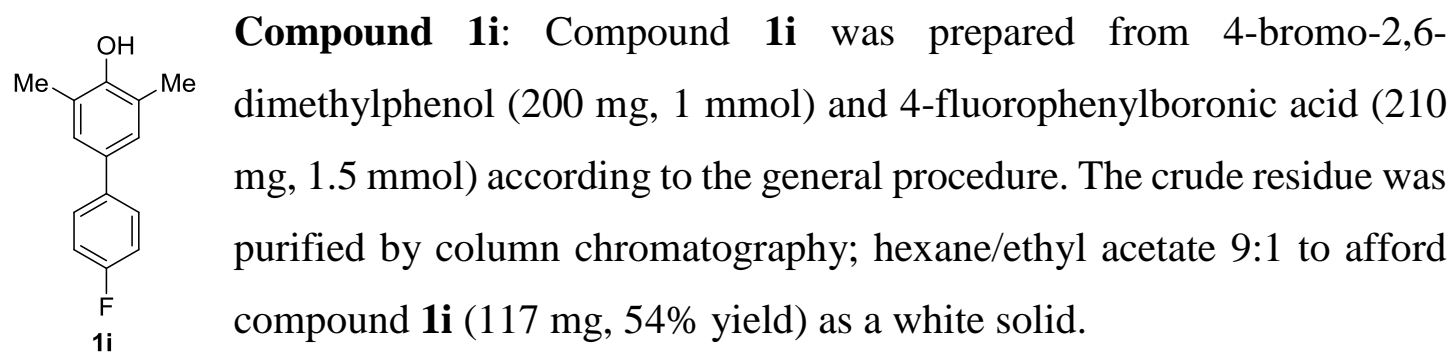

Characterization data of compound 1i: ${ }^{1} \mathrm{H}$ NMR $\left(500 \mathrm{MHz}, \mathrm{CDCl}_{3}\right) \delta 7.48(\mathrm{~m}, 2 \mathrm{H})$, $7.17(\mathrm{~s}, 2 \mathrm{H}), 7.08(\mathrm{~m}, 2 \mathrm{H}), 2.31(\mathrm{~s}, 6 \mathrm{H}) ;{ }^{13} \mathrm{C} \mathrm{NMR}\left(125 \mathrm{MHz}, \mathrm{CDCl}_{3}\right) \delta 163.1,152.0$, 137.4, 132.6, 128.4, 127.4, 123.5, 115.6, 16.2; $\left.{ }^{19} \mathrm{~F} \mathrm{NMR} \mathrm{(377} \mathrm{MHz,} \mathrm{CDCl}_{3}\right) \delta$-117.03; HRMS (ESI): $m / z$ calcd for $\mathrm{C}_{14} \mathrm{H}_{14} \mathrm{FO}[\mathrm{M}+\mathrm{H}]^{+} 217.1023$, found 217.1042.

\subsection{Sequential cross-coupling products:}

\subsubsection{General procedure:}

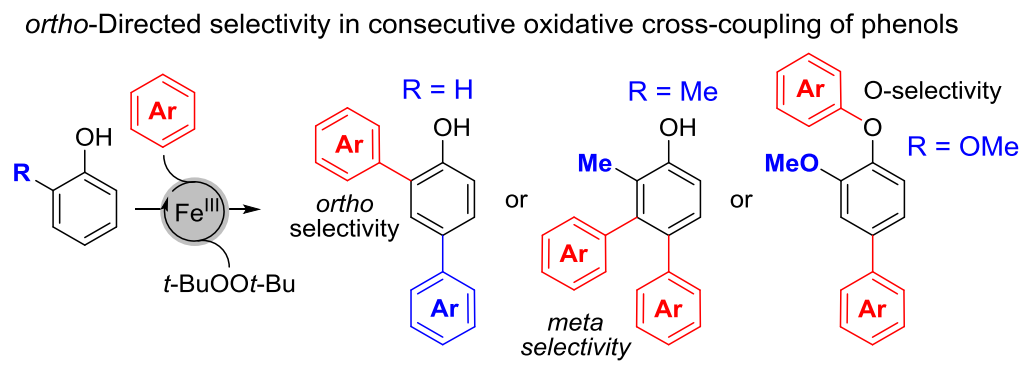

To a stirred solution of phenol derivative (1.0 equiv), arene (1.5-5 equiv) and $\mathrm{FeCl}_{3}$ (15 mol \%) in HFIP (0.5 M), di-t-butylperoxide (1.5-5 equiv) was added drop-wise at room temperature. Upon completion of the sequential oxidative cross-coupling reaction, as indicated by TLC and HPLC analysis, the volatiles were removed under reduced pressure. The crude residue was purified by silica-gel column chromatography affording pure polyarene products. 


\subsubsection{Characterization data:}

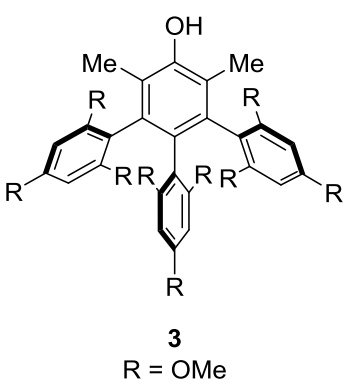

Compound 3: To a mixture of 2,6-dimethylphenol (1a, $31 \mathrm{mg}$, $0.25 \mathrm{mmol}), 1,3,5$-trimethoxybenzene (2a, $126 \mathrm{mg}, 0.75 \mathrm{mmol}$ ) and $\mathrm{FeCl}_{3}(6 \mathrm{mg}, 15 \mathrm{~mol} \%)$ in $\operatorname{HFIP}(0.5 \mathrm{~mL}, 0.5 \mathrm{M})$, di- $t$ butylperoxide $(0.138 \mathrm{~mL}, 0.75 \mathrm{mmol})$ was added drop-wise at room temperature, stirring was continued for $2.5 \mathrm{~h}$ and the volatiles were removed under reduced pressure. The crude residue was purified by column chromatography; hexane/ethyl acetate 4:6 to afford compound 3 (143 mg, 58\% yield) as a white solid.

Compound 3 can also be prepared from $\mathbf{1 b}$ by using above procedure, in $3 \mathrm{~h}$ with $90 \%$ yield.

Characterization data of compound 3: ${ }^{1} \mathrm{H} \mathrm{NMR}\left(400 \mathrm{MHz}, \mathrm{CDCl}_{3}\right) \delta 5.92(\mathrm{~s}, 4 \mathrm{H}), 5.64$ $(\mathrm{s}, 2 \mathrm{H}), 3.75(\mathrm{~s}, 6 \mathrm{H}), 3.65(\mathrm{~s}, 3 \mathrm{H}), 3.52(\mathrm{~s}, 12 \mathrm{H}), 3.33(\mathrm{~s}, 6 \mathrm{H}), 2.01(\mathrm{~s}, 6 \mathrm{H}) ;{ }^{13} \mathrm{C} \mathrm{NMR}$ $\left(100 \mathrm{MHz}, \mathrm{CDCl}_{3}\right) \delta 159.8,158.8,158.7,158.7,150.9,133.3,122.4,112.6,112.5$, 111.3, 89.1, 88.3, 55.3, 55.1, 54.7 54.4, 14.1; HRMS (ESI): $\mathrm{m} / z$ calcd for $\mathrm{C}_{35} \mathrm{H}_{41} \mathrm{O}_{10}$ $[\mathrm{M}+\mathrm{H}]^{+}$621.2694, found 621.2692 .

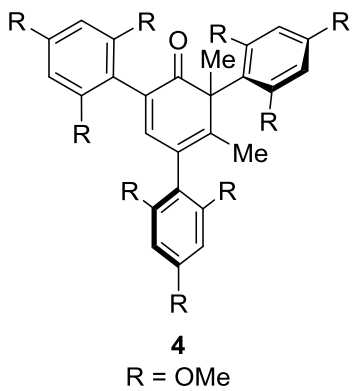

Compound 4: To a mixture of 2,3-dimethylphenol (1c, $31 \mathrm{mg}$, $0.25 \mathrm{mmol}$ ), 1,3,5-trimethoxybenzene (2a, $210 \mathrm{mg}, 1.25 \mathrm{mmol}$ ) and $\mathrm{FeCl}_{3}(6 \mathrm{mg}, 15 \mathrm{~mol} \%)$ in HFIP $(0.5 \mathrm{~mL}, 0.5 \mathrm{M})$, di-tbutylperoxide $(0.230 \mathrm{~mL}, 1.25 \mathrm{mmol})$ was added drop-wise at room temperature, stirring was continued for $7 \mathrm{~h}$ and the volatiles were removed under reduced pressure. The crude residue was purified by column chromatography; hexane/ethyl acetate 4:6 to afford compound 4 (132 $\mathrm{mg}, 85 \%$ yield) as a white solid.

Characterization data of compound 4: ${ }^{1} \mathrm{H}$ NMR $\left(500 \mathrm{MHz}, \mathrm{CDCl}_{3}\right) \delta 6.76(\mathrm{~s}, 1 \mathrm{H}), 6.16-$ 6.12 (m, 5H), 6.05 (s, 1H), 3.82-3.71 (overlapped, 24H), 3.62 (s, 3H), 1.84 (s, 3H), 1.44 (s, 3H); ${ }^{13} \mathrm{C} \mathrm{NMR}\left(125 \mathrm{MHz}, \mathrm{CDCl}_{3}\right) \delta 203.6,160.6,160.4,159.9,159.6,159.1,158.6$, 153.9, 146.6, 125.7, 120.6, 113.7, 112.0, 109.0, 91.6, 91.5, 91.3, 58.8, 56.4, 56.1, 55.8, 55.4, 55.3, 24.3, 17.6; HRMS (ESI): $\mathrm{m} / z$ calcd for $\mathrm{C}_{35} \mathrm{H}_{41} \mathrm{O}_{10}[\mathrm{M}+\mathrm{H}]^{+} 621.2694$, found 621.2697. IR (KBr pellet): 2930(m), 1600(s), 1580(s), $1120(\mathrm{~s}) \mathrm{cm}^{-1}$. 


\section{Synthesis of phenolic intermediates $4 a$ and $4 b$ for the kinetic studies of 1c:}

To a mixture of 2,3-dimethylphenol (1c, $31 \mathrm{mg}, 0.25 \mathrm{mmol}$ ), 1,3,5-trimethoxybenzene (2a, $55 \mathrm{mg}, 0.325 \mathrm{mmol})$ and $\mathrm{FeCl}_{3}(6 \mathrm{mg}, 15 \mathrm{~mol} \%)$ in HFIP $(0.5 \mathrm{~mL}, 0.5 \mathrm{M})$, di-tbutylperoxide $(0.069 \mathrm{~mL}, 0.375 \mathrm{mmol})$ was added drop-wise at room temperature, stirring was continued for $1 \mathrm{~h}$ and the volatiles were removed under reduced pressure. The crude residue was purified by column chromatography; hexane/ethyl acetate 8:2 affording compound $\mathbf{4 a}$ ( $7 \mathrm{mg}, 10 \%$ yield) as a white solid and hexane/ethyl acetate 7:3 compound $4 \mathbf{b}$ (29 $\mathrm{mg}, 26 \%$ yield) as a white solid.
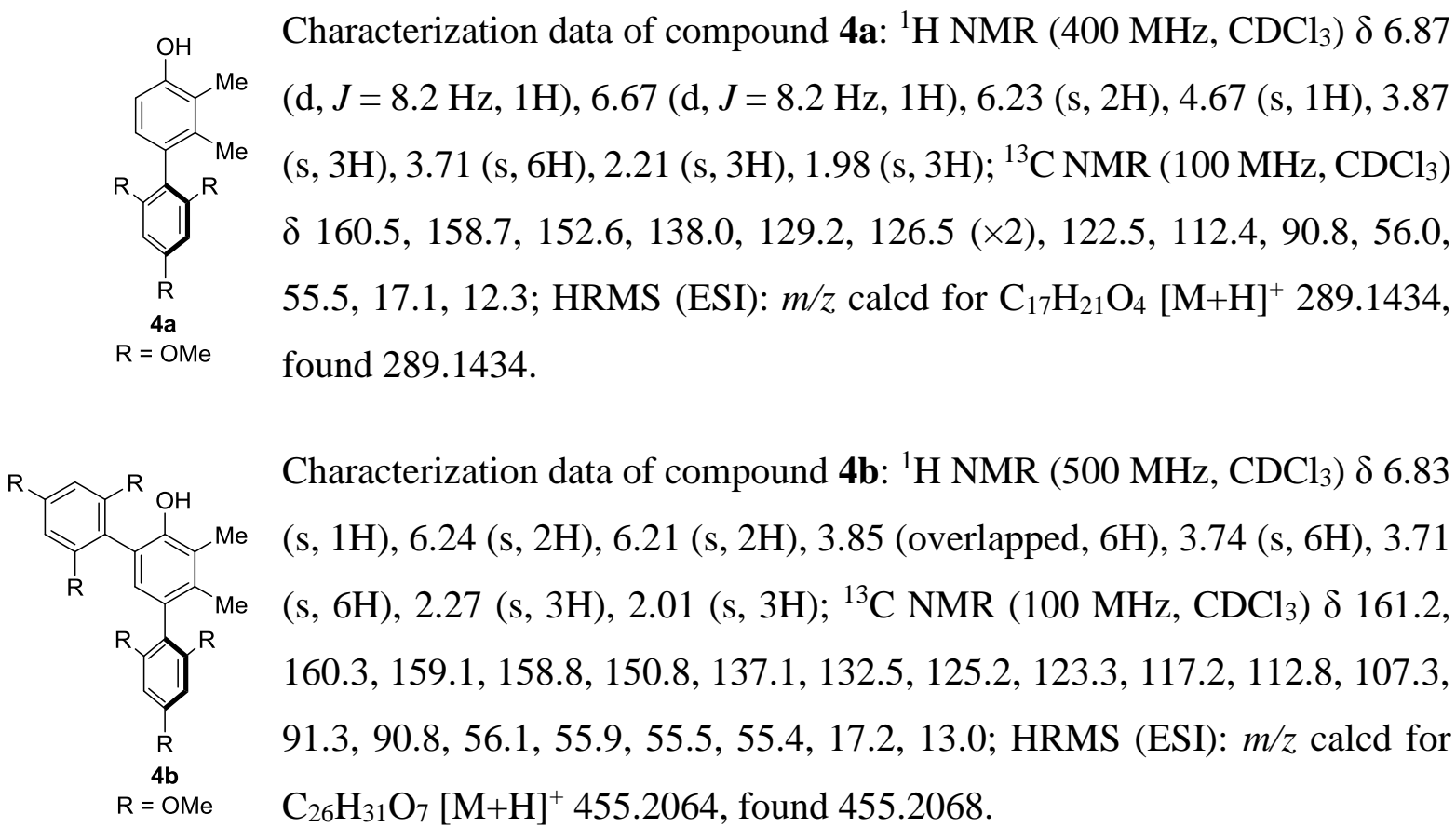

Characterization data of compound $4 \mathbf{b}:{ }^{1} \mathrm{H}$ NMR $\left(500 \mathrm{MHz}, \mathrm{CDCl}_{3}\right) \delta 6.83$ (s, 1H), 6.24 (s, 2H), 6.21 (s, 2H), 3.85 (overlapped, 6H), 3.74 (s, 6H), 3.71 $(\mathrm{s}, 6 \mathrm{H}), 2.27(\mathrm{~s}, 3 \mathrm{H}), 2.01(\mathrm{~s}, 3 \mathrm{H}) ;{ }^{13} \mathrm{C} \mathrm{NMR}\left(100 \mathrm{MHz}, \mathrm{CDCl}_{3}\right) \delta 161.2$, $160.3,159.1,158.8,150.8,137.1,132.5,125.2,123.3,117.2,112.8,107.3$, 91.3, 90.8, 56.1, 55.9, 55.5, 55.4, 17.2, 13.0; HRMS (ESI): $\mathrm{m} / z$ calcd for $\mathrm{C}_{26} \mathrm{H}_{31} \mathrm{O}_{7}[\mathrm{M}+\mathrm{H}]^{+}$455.2064, found 455.2068. 


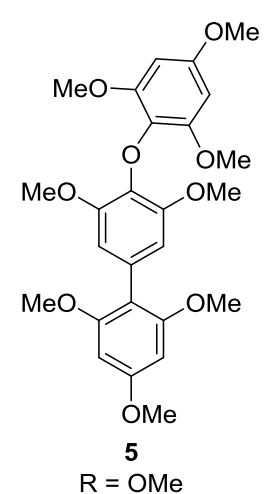

Compound 5: To a mixture of 2,6-dimethoxyphenol (1d, 39 mg, 0.25 mmol), 1,3,5-trimethoxybenzene (2a, $210 \mathrm{mg}, 1.25 \mathrm{mmol})$ and $\mathrm{FeCl}_{3}(6 \mathrm{mg}, 15 \mathrm{~mol} \%)$ in HFIP $(0.5 \mathrm{~mL}, 0.5 \mathrm{M})$, di-t-butylperoxide $(0.230 \mathrm{~mL}, 1.25 \mathrm{mmol})$ was added drop-wise at room temperature, stirring was continued for $3 \mathrm{~h}$ and the volatiles were removed under reduced pressure. The crude residue was purified by column chromatography; hexane/ethyl acetate 6:4 to afford compound 5 (83 $\mathrm{mg}, 68 \%$ yield) as a white solid.

Characterization data of compound 5: ${ }^{1} \mathrm{H}$ NMR $\left(400 \mathrm{MHz}, \mathrm{CDCl}_{3}\right) \delta 6.53(\mathrm{~s}, 2 \mathrm{H}), 6.21$ (s, 2H), 6.15 (s, 2H), $3.84(\mathrm{~s}, 3 \mathrm{H}), 3.77(\mathrm{~s}, 3 \mathrm{H}), 3.72(\mathrm{~s}, 6 \mathrm{H}), 3.69(\mathrm{~s}, 12 \mathrm{H}) ;{ }^{13} \mathrm{C} \mathrm{NMR}$ $\left(100 \mathrm{MHz}, \mathrm{CDCl}_{3}\right) \delta 160.4,158.5,155.4,152.4,150.9,136.5,132.4,128.0,113.0$, 109.8, 92.6, 91.4, 56.9, 56.7, 56.1, 55.5, 55.4; HRMS (ESI): $\mathrm{m} / z$ calcd for $\mathrm{C}_{26} \mathrm{H}_{31} \mathrm{O}_{9}$ $[\mathrm{M}+\mathrm{H}]^{+}$487.1963, found 487.1965. IR (KBr pellet): 2930(m), 1590 (s), 1460(s), $1220(\mathrm{~s}), 1120(\mathrm{~s}) \mathrm{cm}^{-1}$.

\section{Compounds 6 and 7:}

To a mixture of $\mathbf{1 e}(28 \mathrm{mg}, 0.09 \mathrm{mmol})$, 1,3,5-trimethoxybenzene (2a, $50 \mathrm{mg}, 0.30$ $\mathrm{mmol}$ ) and $\mathrm{FeCl}_{3}(2 \mathrm{mg}, 15 \mathrm{~mol} \%)$ in $\operatorname{HFIP}(0.2 \mathrm{~mL}, 0.5 \mathrm{M})$, di-t-butylperoxide $(0.055$ $\mathrm{mL}, 0.30 \mathrm{mmol}$ ) was added drop-wise at room temperature, stirring was continued for $18 \mathrm{~h}$ and the volatiles were removed under reduced pressure. The crude residue was purified by column chromatography; hexane/ethyl acetate 1:1 to afford compound 6 (20 mg, 33\% yield) as a white solid and hexane/ethyl acetate 4:6 to afford compound 7 (25 mg, $44 \%$ yield) as a white solid.

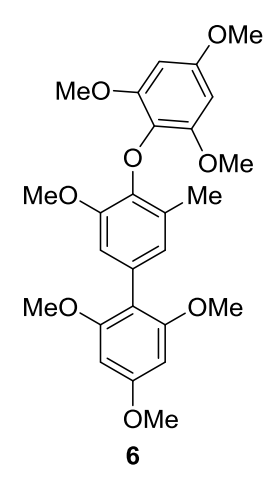

Characterization data of compound 6: ${ }^{1} \mathrm{H}$ NMR $\left(400 \mathrm{MHz}, \mathrm{CDCl}_{3}\right) \delta$ $6.69(\mathrm{~s}, 1 \mathrm{H}), 6.68(\mathrm{~s}, 1 \mathrm{H}), 6.21(\mathrm{~s}, 2 \mathrm{H}), 6.16(\mathrm{~s}, 2 \mathrm{H}), 3.85(\mathrm{~s}, 3 \mathrm{H}), 3.78$ $(\mathrm{s}, 3 \mathrm{H}), 3.71(\mathrm{~s}, 6 \mathrm{H}), 3.69(\mathrm{~s}, 6 \mathrm{H}), 3.61(\mathrm{~s}, 3 \mathrm{H}), 2.27(\mathrm{~s}, 3 \mathrm{H}) ;{ }^{13} \mathrm{C}$ NMR $\left(100 \mathrm{MHz}, \mathrm{CDCl}_{3}\right) \delta 160.3,158.6,155.5,152.5,150.4,145.4$, 132.3, 129.4, 128.3, 126.0, 114.7, 113.1, 92.7, 91.4, 56.8, 56.7, 56.1, 55.6, 55.5, 16.9; HRMS (ESI): $m / z$ calcd for $\mathrm{C}_{26} \mathrm{H}_{31} \mathrm{O}_{8}[\mathrm{M}+\mathrm{H}]^{+}$ 471.2013, found 471.2018 . 

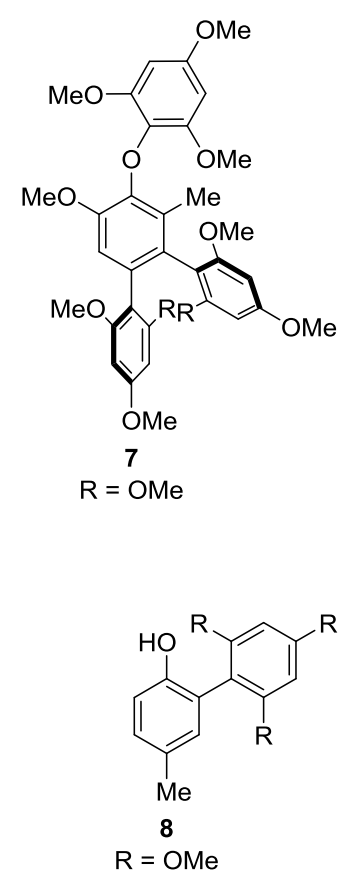

Characterization data of compound 7: ${ }^{1} \mathrm{H}$ NMR $\left(400 \mathrm{MHz}, \mathrm{CDCl}_{3}\right) \delta$ $6.66(\mathrm{~s}, 1 \mathrm{H}), 6.17$ (s, 2H), 5.95 (overlapped, 4H), 3.77 (s, 3H), 3.75 (s, $6 \mathrm{H}), 3.69(\mathrm{~s}, 6 \mathrm{H}), 3.60$ (s, 3H), 3.51 (overlapped, 12H), $2.01(\mathrm{~s}, 3 \mathrm{H})$; ${ }^{13} \mathrm{C}$ NMR $\left(100 \mathrm{MHz}, \mathrm{CDCl}_{3}\right) \delta 159.8,159.7,158.8,158.5,155.2$, $152.5,149.3,145.5,133.5,130.9,129.8,128.3,114.8,113.2,111.4$ 93.7, 90.0, 89.9, 57.1, 56.6, 55.6, $55.3(\times 2), 14.3$; HRMS (ESI): $m / z$ calcd for $\mathrm{C}_{35} \mathrm{H}_{41} \mathrm{O}_{11}[\mathrm{M}+\mathrm{H}]^{+}$637.2643, found 637.2657.

Compound 8: To a mixture of $p$-cresol (27 mg, $0.25 \mathrm{mmol}), 1,3,5-$ trimethoxybenzene (2a, $126 \mathrm{mg}, 0.75 \mathrm{mmol})$ and $\mathrm{FeCl}_{3}(6 \mathrm{mg}, 15 \mathrm{~mol}$ $\%)$ in HFIP $(0.5 \mathrm{~mL}, 0.5 \mathrm{M})$, di-t-butylperoxide $(0.138 \mathrm{~mL}, 0.75 \mathrm{mmol})$ was added drop-wise at room temperature, stirring was continued for 4 $\mathrm{h}$ and the volatiles were removed under reduced pressure. The crude residue was purified by column chromatography; hexane/ethyl acetate 6:4 to afford compound 8 (28 $\mathrm{mg}, 41 \%$ yield) as a white solid.

Characterization data of compound 8: ${ }^{1} \mathrm{H}$ NMR $\left(500 \mathrm{MHz}, \mathrm{CDCl}_{3}\right) \delta 7.07(\mathrm{~d}, J=7.7$ Hz, 1H), 6.99 (s, 1H), 6.92 (d, J= $7.7 \mathrm{~Hz}, 1 \mathrm{H}), 6.27$ (s, 2H), 5.10 (s, 1H), 3.88 (s, 3H), $3.76(\mathrm{~s}, 6 \mathrm{H}), 2.31(\mathrm{~s}, 3 \mathrm{H}) ;{ }^{13} \mathrm{C} \mathrm{NMR}\left(125 \mathrm{MHz}, \mathrm{CDCl}_{3}\right) \delta 161.6,158.9,151.6,132.9$, 129.6, 129.2, 120.6, 115.9, 91.4, 56.2, 55.5, 20.8; HRMS (ESI): $\mathrm{m} / z$ calcd for $\mathrm{C}_{16} \mathrm{H}_{19} \mathrm{O}_{4}$ $[\mathrm{M}+\mathrm{H}]^{+} 275.1278$, found 275.1283. 


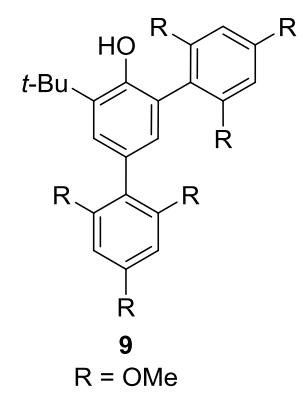

Compound 9: To a mixture of 2-tert-butyl phenol (38 mg, 0.25 mmol), 1,3,5-trimethoxybenzene (2a, $210 \mathrm{mg}, 1.25 \mathrm{mmol})$ and $\mathrm{FeCl}_{3}(6 \mathrm{mg}, 15 \mathrm{~mol} \%)$ in $\operatorname{HFIP}(0.5 \mathrm{~mL}, 0.5 \mathrm{M})$, di-t-butylperoxide $(0.230 \mathrm{~mL}, 1.25 \mathrm{mmol})$ was added drop-wise at room temperature, stirring was continued for $20 \mathrm{~h}$ and the volatiles were removed under reduced pressure. The crude residue was purified by column chromatography; hexane/ethyl acetate 8:2 to afford compound 9 (51 mg, $44 \%$ yield) as a white solid.

Characterization data of compound 9: ${ }^{1} \mathrm{H}$ NMR $\left(400 \mathrm{MHz}, \mathrm{CDCl}_{3}\right) \delta 7.29(\mathrm{~d}, J=1.9$ $\mathrm{Hz}, 1 \mathrm{H}), 7.06$ (d, J=1.9 Hz, 1H), 6.26 (s, 2H), 6.23 (s, 2H), 3.86 (s, 3H), 3.85 (s, 3H), $3.74(\mathrm{~s}, 6 \mathrm{H}), 3.73$ (s, 6H), 1.45 (s, 9H); $\left.{ }^{13} \mathrm{C} \mathrm{NMR} \mathrm{(100} \mathrm{MHz,} \mathrm{CDCl}_{3}\right) \delta 161.3,159.8$, 159.2, 158.6, 151.2, 134.9, 133.2, 129.6, 123.9, 120.5, 113.4, 107.6, 91.7, 91.5, 56.2, 56.1, 55.5, 55.5, 35.0, 30.1; HRMS (ESI): $m / z$ calcd for $\mathrm{C}_{28} \mathrm{H}_{35} \mathrm{O}_{7}[\mathrm{M}+\mathrm{H}]^{+} 483.2377$, found 483.2374 .

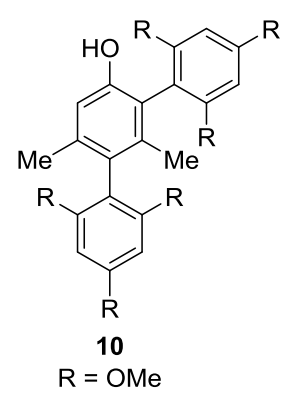

Compound 10: To a mixture of 3,5-dimethylphenol (31 mg, 0.25 mmol), 1,3,5-trimethoxybenzene (2a, $126 \mathrm{mg}, 0.75 \mathrm{mmol})$ and $\mathrm{FeCl}_{3}(6 \mathrm{mg}, 15 \mathrm{~mol} \%)$ in $\mathrm{HFIP}(0.5 \mathrm{~mL}, 0.5 \mathrm{M})$, di-t-butylperoxide $(0.138 \mathrm{~mL}, 0.75 \mathrm{mmol})$ was added drop-wise at room temperature, stirring was continued for $4 \mathrm{~h}$ and the volatiles were removed under reduced pressure. The crude residue was purified by column chromatography; hexane/ethyl acetate $6: 4$ compound $\mathbf{1 0}(40 \mathrm{mg}, 35 \%$ yield) as a white solid.

Characterization data of compound 10: ${ }^{1} \mathrm{H}$ NMR $\left(500 \mathrm{MHz}, \mathrm{CDCl}_{3}\right) \delta 6.78(\mathrm{~s}, 1 \mathrm{H})$, $6.26(\mathrm{~s}, 2 \mathrm{H}), 6.24$ (s, 2H), 3.87 (overlapped, 6H), 3.73 (s, 6H), 3.70 (s, 6H), 2.01 (s, 3H), $1.65(\mathrm{~s}, 3 \mathrm{H}) ;{ }^{13} \mathrm{C} \mathrm{NMR}\left(125 \mathrm{MHz}, \mathrm{CDCl}_{3}\right) \delta 161.7,160.4,159.5,158.6,152.3$, 138.7, 138.0, 125.9, 117.7, 113.7, 111.5, 105.3, 91.4, 91.1, 56.1, 56.0, 55.5, 55.4, 20.5, 17.6; HRMS (ESI): $m / z$ calcd for $\mathrm{C}_{26} \mathrm{H}_{31} \mathrm{O}_{7}[\mathrm{M}+\mathrm{H}]^{+} 455.2064$, found 455.2059. 


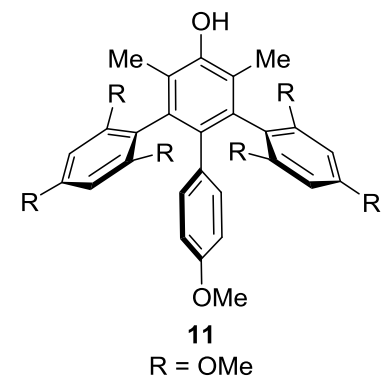

column chromatography; hexane/ethyl acetate 1:1 to afford compound $\mathbf{1 1}$ (134 mg, $96 \%$ yield) as a white solid.

Characterization data of compound 11: ${ }^{1} \mathrm{H} \mathrm{NMR}\left(400 \mathrm{MHz}, \mathrm{CDCl}_{3}\right) \delta 6.78-6.61(\mathrm{~m}$, $2 \mathrm{H}), 6.45-6.24(\mathrm{~m}, 2 \mathrm{H}), 5.94(\mathrm{~s}, 4 \mathrm{H}), 4.71(\mathrm{~s}, 1 \mathrm{H}), 3.74(\mathrm{~s}, 6 \mathrm{H}), 3.61(\mathrm{~s}, 3 \mathrm{H}), 3.58(\mathrm{~s}$, 12H), 2.01 (s, 6H); ${ }^{13} \mathrm{C}$ NMR (100 MHz, $\left.\mathrm{CDCl}_{3}\right) \delta 160.3,158.1,156.9,150.8,136.4$, 134.9, 131.9, 130.3, 122.2 111.5, 110.8, 90.0, 55.4, 55.3, 55.0, 13.6; HRMS (ESI): $\mathrm{m} / \mathrm{z}$ calcd for $\mathrm{C}_{33} \mathrm{H}_{37} \mathrm{O}_{8}[\mathrm{M}+\mathrm{H}]^{+}$561.2483, found 561.2479. IR ( $\mathrm{KBr}$ pellet): 3420(m, broad), 2920(m), 1490(s), 1220(s), 1120(s) $\mathrm{cm}^{-1}$.

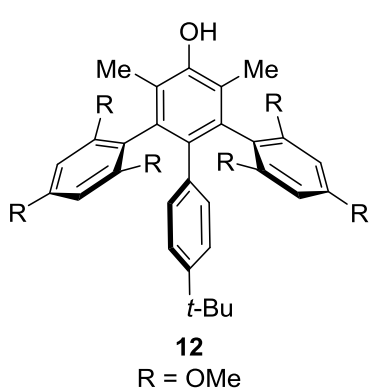

Compound 12: To a mixture of $\mathbf{1 h}(64 \mathrm{mg}, 0.25 \mathrm{mmol}), 1,3,5-$ trimethoxybenzene (2a, $126 \mathrm{mg}, 0.75 \mathrm{mmol})$ and $\mathrm{FeCl}_{3}(6 \mathrm{mg}$, $15 \mathrm{~mol} \%)$ in HFIP $(0.5 \mathrm{~mL}, 0.5 \mathrm{M})$, di-t-butylperoxide $(0.138$ $\mathrm{mL}, 0.75 \mathrm{mmol}$ ) was added drop-wise at room temperature, stirring was continued for $18 \mathrm{~h}$ and the volatiles were removed under reduced pressure. The crude residue was purified by column chromatography; hexane/ethyl acetate 7:3 to afford compound $\mathbf{1 2}$ (86 mg, 60\% yield) as a white solid.

Characterization data of compound 12: ${ }^{1} \mathrm{H}$ NMR $\left(400 \mathrm{MHz}, \mathrm{CDCl}_{3}\right) \delta 6.77-6.73(\mathrm{~m}$, 2H), $6.63-6.60(\mathrm{~m}, 2 \mathrm{H}), 5.92(\mathrm{~s}, 3 \mathrm{H}), 3.72(\mathrm{~s}, 6 \mathrm{H}), 3.55(\mathrm{~s}, 12 \mathrm{H}), 2.04(\mathrm{~s}, 6 \mathrm{H}), 1.11$ $(\mathrm{s}, 9 \mathrm{H}) ;{ }^{13} \mathrm{C} \mathrm{NMR}\left(100 \mathrm{MHz}, \mathrm{CDCl}_{3}\right) \delta 160.4,158.3,150.8,147.0,139.0,136.8,131.7$, 129.1, 122.3, 121.8, 111.6, 89.9, 55.4, 55.3, 31.4, 13.6; HRMS (ESI): $\mathrm{m} / \mathrm{z}$ calcd for $\mathrm{C}_{36} \mathrm{H}_{43} \mathrm{O}_{7}[\mathrm{M}+\mathrm{H}]^{+}$587.3003, found 587.3016. 


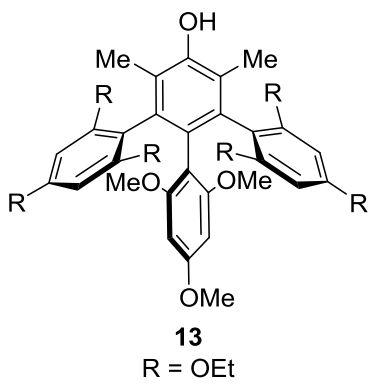

purified by column chromatography; hexane/ethyl acetate 6:4 to afford compound 13 (41 mg, 85\% yield) as a white solid.

Characterization data of compound 13: ${ }^{1} \mathrm{H}$ NMR (400 MHz, $\left.\mathrm{CDCl}_{3}\right) \delta 5.92(\mathrm{~s}, 4 \mathrm{H})$, $5.60(\mathrm{~s}, 2 \mathrm{H}), 4.65(\mathrm{~s}, 1 \mathrm{H}), 3.93(\mathrm{q}, J=6.9 \mathrm{~Hz}, 4 \mathrm{H}), 3.78(\mathrm{~m}, 8 \mathrm{H}), 3.63(\mathrm{~s}, 3 \mathrm{H}), 3.26$ (s, $6 \mathrm{H}), 2.04(\mathrm{~s}, 6 \mathrm{H}), 1.36(\mathrm{t}, J=6.9 \mathrm{~Hz}, 6 \mathrm{H}), 1.19(\mathrm{t}, J=6.9 \mathrm{~Hz}, 12 \mathrm{H}) ;{ }^{13} \mathrm{C}$ NMR $(100$ $\left.\mathrm{MHz}, \mathrm{CDCl}_{3}\right) \delta 167.1,158.8,158.7,158.1,150.6,133.8,122.1,112.5,112.4,91.6$, 88.3, 63.6, 63.3, 55.1, 54.0, 15.0, 14.8, 14.2; HRMS (ESI): $\mathrm{m} / z$ calcd for $\mathrm{C}_{41} \mathrm{H}_{53} \mathrm{O}_{10}$ $[\mathrm{M}+\mathrm{H}]^{+}$705.3633, found 705.3644.

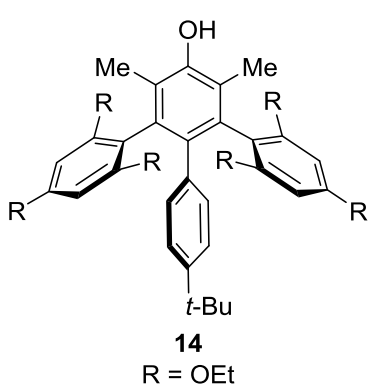

Compound 14: To a mixture of $\mathbf{1 h}(29 \mathrm{mg}, 0.125 \mathrm{mmol})$, 1,3,5-triethoxybenzene (2b, $79 \mathrm{mg}, 0.375 \mathrm{mmol})$ and $\mathrm{FeCl}_{3}(3$ $\mathrm{mg}, 15 \mathrm{~mol} \%)$ in HFIP $(0.5 \mathrm{~mL}, 0.5 \mathrm{M})$, di-t-butylperoxide (0.069 mL, $0.375 \mathrm{mmol})$ was added drop-wise at room temperature, stirring was continued for $24 \mathrm{~h}$ and the volatiles were removed under reduced pressure. The crude residue was purified by column chromatography; hexane/ethyl acetate $8: 2$ to afford compound 14 (18 $\mathrm{mg}, 21 \%$ yield) as a white solid.

Characterization data of compound 14: ${ }^{1} \mathrm{H}$ NMR (500 MHz, $\left.\mathrm{CDCl}_{3}\right) \delta 6.69(\mathrm{~s}, 4 \mathrm{H})$, $5.89(\mathrm{~s}, 4 \mathrm{H}), 3.91(\mathrm{q}, J=6.9 \mathrm{~Hz}, 4 \mathrm{H}), 3.84(\mathrm{~m}, 4 \mathrm{H}), 3.64(\mathrm{~m}, 4 \mathrm{H}), 2.01(\mathrm{~s}, 6 \mathrm{H}), 1.33(\mathrm{t}$, $J=6.9 \mathrm{~Hz}, 6 \mathrm{H}), 1.19(\mathrm{t}, J=6.9 \mathrm{~Hz}, 12 \mathrm{H}), 1.08(\mathrm{~s}, 9 \mathrm{H}) ;{ }^{13} \mathrm{C} \mathrm{NMR}\left(125 \mathrm{MHz}, \mathrm{CDCl}_{3}\right) \delta$ $159.2,157.7,150.3,146.7,139.1,136.3,131.9,129.2,121.8,121.4,113.0,91.8,63.5$, 63.5, 31.4, 14.9, 13.5; HRMS (ESI): $m / z$ calcd for $\mathrm{C}_{42} \mathrm{H}_{55} \mathrm{O}_{7}[\mathrm{M}+\mathrm{H}]^{+} 671.3942$, found 671.3953. 


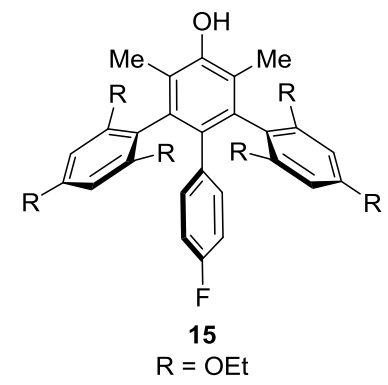

column chromatography; hexane/ethyl acetate 8:2 to afford compound $\mathbf{1 5}$ (18 mg, 23\% yield) as a white solid.

Characterization data of compound 15: ${ }^{1} \mathrm{H} \mathrm{NMR}\left(500 \mathrm{MHz}, \mathrm{CDCl}_{3}\right) \delta 6.78(\mathrm{dd}, J=6.9$ $\mathrm{Hz}, J=2.6 \mathrm{~Hz}, 2 \mathrm{H}), 6.42(\mathrm{t}, J=9 \mathrm{~Hz}, 2 \mathrm{H}), 5.91(\mathrm{~s}, 4 \mathrm{H}), 3.92(\mathrm{q}, J=7.0 \mathrm{~Hz}, 4 \mathrm{H}), 3.85$ $(\mathrm{m}, 4 \mathrm{H}), 3.68(\mathrm{~m}, 4 \mathrm{H}), 2.00(\mathrm{~s}, 6 \mathrm{H}), 1.36(\mathrm{t}, J=7.0 \mathrm{~Hz}, 6 \mathrm{H}), 1.20(\mathrm{t}, J=7.0 \mathrm{~Hz}, 12 \mathrm{H})$; ${ }^{13} \mathrm{C}$ NMR $\left(125 \mathrm{MHz}, \mathrm{CDCl}_{3}\right) \delta 159.5,157.5,150.5,138.4,135.2,132.0,130.8,130.8$, 121.4, 112.1, 112.0, 91.6, 63.5, 63.4, 15.0, 13.4; ${ }^{19} \mathrm{~F}$ NMR $\left(377 \mathrm{MHz}, \mathrm{CDCl}_{3}\right) \delta$ 119.48; HRMS (ESI): $m / z$ calcd for $\mathrm{C}_{38} \mathrm{H}_{46} \mathrm{FO}_{7}[\mathrm{M}+\mathrm{H}]^{+}$633.3222, found 633.3226 .

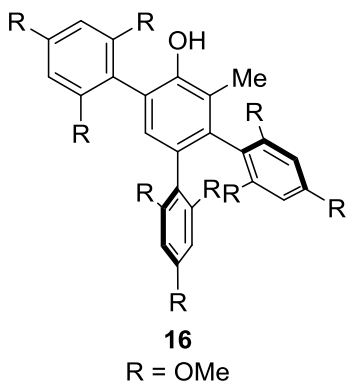

Compound 16: To a mixture of $o$-cresol $(27 \mathrm{mg}, 0.25 \mathrm{mmol})$, 1,3,5-trimethoxybenzene (2a, $42 \mathrm{mg}, 0.25 \mathrm{mmol})$ and $\mathrm{FeCl}_{3}(6$ $\mathrm{mg}, 15 \mathrm{~mol} \%)$ in HFIP $(0.5 \mathrm{~mL}, 0.5 \mathrm{M})$, di-t-butylperoxide $(0.046 \mathrm{~mL}, 0.25 \mathrm{mmol})$ was added drop-wise at room temperature, stirring was continued for $23 \mathrm{~h}$, while every 4 ours another portion of $\mathbf{2 a}$ and di-t-butylperoxide were added up to 3 equivalents in total. The volatiles were removed under reduced pressure. The crude residue was purified by column chromatography; hexane/ethyl acetate 4:6 to afford compound 16 (47 $\mathrm{mg}, 32 \%$ yield) as a white solid.

Characterization data of compound 16: ${ }^{1} \mathrm{H}$ NMR (400 MHz, $\left.\mathrm{CDCl}_{3}\right) \delta 6.97(\mathrm{~s}, 1 \mathrm{H})$, $6.25(\mathrm{~s}, 2 \mathrm{H}), 5.98(\mathrm{~s}, 2 \mathrm{H}), 5.95(\mathrm{~s}, 2 \mathrm{H}), 3.86(\mathrm{~s}, 3 \mathrm{H}), 3.78$ (overlapped, 9H), 3.75 (s, $3 \mathrm{H}), 3.57(\mathrm{~s}, 6 \mathrm{H}), 3.54(\mathrm{~s}, 6 \mathrm{H}), 2.06(\mathrm{~s}, 3 \mathrm{H}) ;{ }^{13} \mathrm{C} \mathrm{NMR}\left(100 \mathrm{MHz}, \mathrm{CDCl}_{3}\right) \delta 161.0$, $159.9,159.4,158.9,158.8,158.6,150.8,135.0,133.5,126.5,125.4,118.4,113.6$, 111.3, 108.1, 91.5, 90.1, 89.8, 56.0, 55.5, 55.3, 55.2, 14.1; HRMS (ESI): $\mathrm{m} / z$ calcd for $\mathrm{C}_{34} \mathrm{H}_{39} \mathrm{O}_{10}[\mathrm{M}+\mathrm{H}]^{+}$607.2538, found 607.2521. 


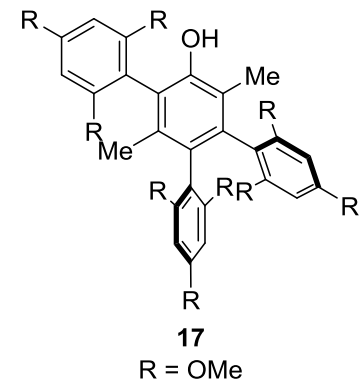

Compound 17: To a mixture of 2,5-dimethylphenol (31 mg, $0.25 \mathrm{mmol}$ ), 1,3,5-trimethoxybenzene (2a, $126 \mathrm{mg}, 0.75 \mathrm{mmol}$ ) and $\mathrm{FeCl}_{3}(6 \mathrm{mg}, 15 \mathrm{~mol} \%)$ in TFE/HFIP $(1: 1,0.5 \mathrm{~mL}, 0.5 \mathrm{M})$, di- $t$-butylperoxide $(0.138 \mathrm{~mL}, 0.75 \mathrm{mmol})$ was added drop-wise at room temperature, stirring was continued for $5 \mathrm{~h}$ and the volatiles were removed under reduced pressure. The crude residue was purified by column chromatography; hexane/ethyl acetate 4:6 to afford compound 17 (84 mg, 54\% yield) as a white solid.

Characterization data of compound 17: ${ }^{1} \mathrm{H}$ NMR $\left(500 \mathrm{MHz}, \mathrm{CDCl}_{3}\right) \delta 6.26(\mathrm{~s}, 2 \mathrm{H})$, $5.95(\mathrm{~s}, 2 \mathrm{H}), 5.93(\mathrm{~s}, 2 \mathrm{H}), 5.28(\mathrm{~s}, 1 \mathrm{H}), 3.87$ (s, 3H), 3.76 (overlapped, 9H), 3.74 (s, $3 \mathrm{H}), 3.56(\mathrm{~s}, 6 \mathrm{H}), 3.54(\mathrm{~s}, 6 \mathrm{H}), 1.99(\mathrm{~s}, 3 \mathrm{H}), 1.67$ (s, 3H); ${ }^{13} \mathrm{C} \mathrm{NMR}\left(125 \mathrm{MHz}, \mathrm{CDCl}_{3}\right)$ $\delta 161.6,159.8,159.5,159.5,158.8,158.5,150.5,136.1,135.1,126.8,121.3,118.6$, $112.4,111.5,106.1,91.3,89.9,89.6,55.9,55.5,55.3,55.3,55.2,53.6,17.9,13.7$; HRMS (ESI): $m / z$ calcd for $\mathrm{C}_{35} \mathrm{H}_{41} \mathrm{O}_{10}[\mathrm{M}+\mathrm{H}]^{+} 621.2694$, found 621.2686.

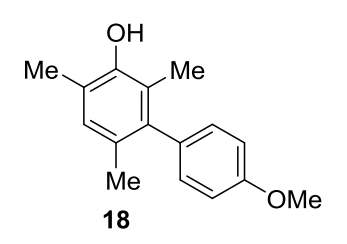

Compound 18: To a mixture of 2,4,6-trimethylphenol (34 mg, $0.25 \mathrm{mmol}$ ), anisole (2c, $81 \mathrm{mg}, 0.75 \mathrm{mmol})$ and $\mathrm{FeCl}_{3}(6 \mathrm{mg}, 15$ mol \%) in HFIP $(0.5 \mathrm{~mL}, 0.5 \mathrm{M})$, di-t-butylperoxide $(0.138 \mathrm{~mL}$, $0.75 \mathrm{mmol}$ ) was added drop-wise at room temperature, stirring was continued for $7 \mathrm{~h}$ and the volatiles were removed under reduced pressure. The crude residue was purified by column chromatography; hexane/diethyl ether 19:1 to afford compound 18 (19 $\mathrm{mg}, 32 \%$ yield) as an orange liquid.

Characterization data of compound 18: ${ }^{1} \mathrm{H}$ NMR (400 MHz, $\left.\mathrm{CDCl}_{3}\right) \delta 7.06-7.04(\mathrm{~m}$, 1H), $7.04-7.02(\mathrm{~m}, 1 \mathrm{H}), 6.98-6.96(\mathrm{~m}, 1 \mathrm{H}), 6.96-6.93(\mathrm{~m}, 1 \mathrm{H}), 6.90(\mathrm{~s}, 1 \mathrm{H}), 4.55$ (s, 1H), $3.86(\mathrm{~s}, 3 \mathrm{H}), 2.28(\mathrm{~s}, 3 \mathrm{H}), 1.95(\mathrm{~s}, 6 \mathrm{H}) ;{ }^{13} \mathrm{C} \mathrm{NMR}\left(100 \mathrm{MHz}, \mathrm{CDCl}_{3}\right) \delta 158.4$, $150.2,140.5,133.5,130.5,129.3,128.2$, 122.0, 121.7, 113.9, 55.4, 29.9, 20.3, 16.0, 13.6; HRMS (ESI): $m / z$ calcd for $\mathrm{C}_{16} \mathrm{H}_{19} \mathrm{O}_{2}[\mathrm{M}+\mathrm{H}]^{+}$243.1385, found 243.1380. 


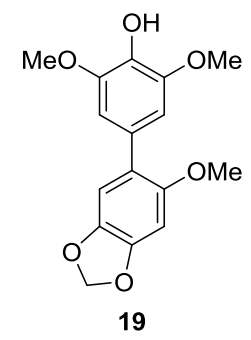

Compound 19: To a mixture of 2,6-dimethoxyphenol (1d, $39 \mathrm{mg}, 0.25$ mmol), 5- methoxy-1,3-benzodioxole (2d, $114 \mathrm{mg}, 0.75 \mathrm{mmol})$ and $\mathrm{FeCl}_{3}(6 \mathrm{mg}, 15 \mathrm{~mol} \%)$ in HFIP $(0.5 \mathrm{~mL}, 0.5 \mathrm{M})$, di-t-butylperoxide $(0.138 \mathrm{~mL}, 0.75 \mathrm{mmol})$ was added drop-wise at room temperature, stirring was continued for $4 \mathrm{~h}$ and the volatiles were removed under reduced pressure. The crude residue was purified by column chromatography; hexane/ethyl acetate 4:1 to afford compound 19 (65 mg, 80\% yield).

Characterization data of compound 19: ${ }^{1} \mathrm{H}$ NMR $\left(400 \mathrm{MHz}, \mathrm{CDCl}_{3}\right) \delta 6.82(\mathrm{~s}, 1 \mathrm{H})$, $6.71(\mathrm{~s}, 2 \mathrm{H}), 6.62(\mathrm{~s}, 1 \mathrm{H}), 5.95(\mathrm{~s}, 2 \mathrm{H}), 5.59(\mathrm{~s}, 1 \mathrm{H}), 3.90(\mathrm{~s}, 6 \mathrm{H}), 3.73(\mathrm{~s}, 3 \mathrm{H}),{ }^{13} \mathrm{C} \mathrm{NMR}$ $\left(100 \mathrm{MHz}, \mathrm{CDCl}_{3}\right) \delta 151.7,147.2,146.7,141.5,133.9,129.6,123.4,110.3,106.5$, 101.3, 95.6, 56.9, 56.4; HRMS (ESI): $m / z$ calcd for $\mathrm{C}_{16} \mathrm{H}_{17} \mathrm{O}_{6}[\mathrm{M}+\mathrm{H}]^{+} 305.0975$, found 305.1020 .

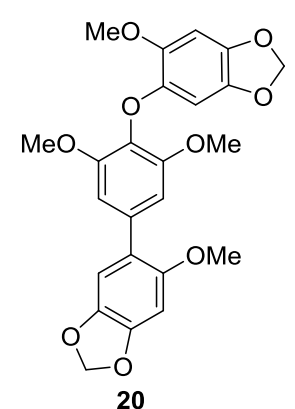

Compound 20: To a mixture of 2,6-dimethoxyphenol (1d, $39 \mathrm{mg}$, $0.25 \mathrm{mmol})$, 5-methoxy-1,3-benzodioxole (2d, $114 \mathrm{mg}, 0.75 \mathrm{mmol})$ and $\mathrm{FeCl}_{3}(6 \mathrm{mg}, 15 \mathrm{~mol} \%)$ in $\operatorname{HFIP}(0.5 \mathrm{~mL}, 0.5 \mathrm{M})$, di-tbutylperoxide $(0.138 \mathrm{~mL}, 0.75 \mathrm{mmol})$ was added drop-wise at room temperature, stirring was continued for $25 \mathrm{~h}$ and the volatiles were removed under reduced pressure. The crude residue was purified by column chromatography (from hexane/ethyl acetate 17:3 to 4:1) affording a mixture of compound 20 (36 mg, 32\% yield) as a brown solid and compound 19 (18 mg, 23\% yield).

Characterization data of compound 20: ${ }^{1} \mathrm{H}$ NMR $\left(400 \mathrm{MHz}, \mathrm{CDCl}_{3}\right) \delta 6.86(\mathrm{~s}, 1 \mathrm{H}), 6.74$ (s, 2H), $6.64(\mathrm{~s}, 1 \mathrm{H}), 6.63(\mathrm{~s}, 1 \mathrm{H}), 6.26(\mathrm{~s}, 1 \mathrm{H}), 5.97(\mathrm{~s}, 2 \mathrm{H}), 5.84(\mathrm{~s}, 2 \mathrm{H}), 3.91(\mathrm{~s}, 3 \mathrm{H})$, 3.78 (s, 6H), 3.75 (s, 3H); $\left.{ }^{13} \mathrm{C} \mathrm{NMR} \mathrm{(100} \mathrm{MHz,} \mathrm{CDCl}_{3}\right) \delta 152.9,151.9,147.7,143.3$, 141.7, 141.3, 140.9, 135.9, 123.2, 110.4, 107.1, 101.5, 101.1, 97.3, 97.1, 95.8, 57.9, 57.0, 56.4, 29.8; HRMS (ESI): $\mathrm{m} / z$ calcd for $\mathrm{C}_{24} \mathrm{H}_{23} \mathrm{O}_{9}[\mathrm{M}+\mathrm{H}]^{+} 455.1337$, found 455.1337. IR (KBr pellet): 2430(m), 1590(s), 1230(s), 1130 (s), 1090(s) $\mathrm{cm}^{-1}$. 


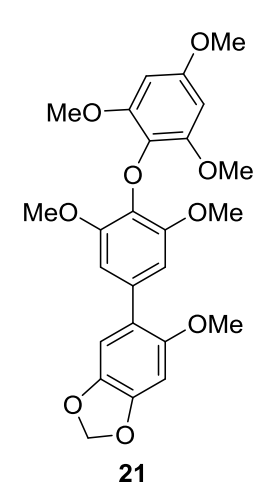

Compound 21: To a mixture of 19 (54 mg, $0.18 \mathrm{mmol}), 1,3,5-$ trimethoxybenzene (2a, $91 \mathrm{mg}, 0.53 \mathrm{mmol})$ and $\mathrm{FeCl}_{3}(43 \mathrm{mg}, 15 \mathrm{~mol}$ $\%)$ in HFIP $(0.4 \mathrm{~mL}, 0.5 \mathrm{M})$, di-t-butylperoxide $(0.100 \mathrm{~mL}, 0.53 \mathrm{mmol})$ was added drop-wise at room temperature, stirring was continued for $5 \mathrm{~h}$ and the volatiles were removed under reduced pressure. The crude residue was purified by column chromatography; hexane/ethyl acetate 4:1 to afford compound 21 (56 $\mathrm{mg}, 67 \%$ yield) as a yellow solid.

Characterization data of compound 21: ${ }^{1} \mathrm{H} \mathrm{NMR}\left(400 \mathrm{MHz}, \mathrm{CDCl}_{3}\right) \delta 6.83(\mathrm{~s}, 1 \mathrm{H}), 6.69$ (s, 2H), 6.60 (s, 1H), 6.14 (s, 2H), 5.93 (s, 2H), 3.76 (s, 3H), 3.72 (overlapped, 12H), $3.68(\mathrm{~s}, 3 \mathrm{H}) ;{ }^{13} \mathrm{C} \mathrm{NMR}\left(100 \mathrm{MHz}, \mathrm{CDCl}_{3}\right) \delta 155.5,152.3,151.7,151.1,147.2,141.6$, 136.8, 132.4, 123.5(×2), 110.2, 108.0, 101.3, 96.1, 92.3, 57.1, 56.9, 56.6, 55.5; HRMS (ESI): $m / z$ calcd for $\mathrm{C}_{25} \mathrm{H}_{26} \mathrm{O}_{9} \mathrm{Na}[\mathrm{M}+\mathrm{Na}]^{+} 493.1469$, found 493.1469 .

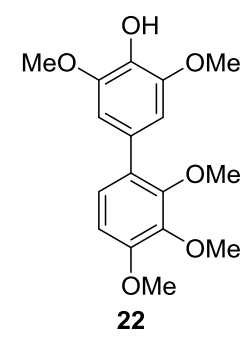

Compound 22: To a mixture of 2,6-dimethoxyphenol (1d, $39 \mathrm{mg}, 0.25$ mmol), 1,2,3-trimethoxybenzene (2e, $126 \mathrm{mg}, 0.75 \mathrm{mmol})$ and $\mathrm{FeCl}_{3}(6$ $\mathrm{mg}, 15 \mathrm{~mol} \%)$ in HFIP $(0.5 \mathrm{~mL}, 0.5 \mathrm{M})$, di-t-butylperoxide $(0.138 \mathrm{~mL}$, $0.75 \mathrm{mmol}$ ) was added drop-wise at room temperature, stirring was continued for $3 \mathrm{~h}$ and the volatiles were removed under reduced pressure. The crude residue was purified by column chromatography; hexane/ethyl acetate $4: 1$ to afford compound 22 (33 $\mathrm{mg}, 40 \%$ yield) as an orange liquid.

Characterization data of compound 22: ${ }^{1} \mathrm{H}$ NMR (400 MHz, $\left.\mathrm{CDCl}_{3}\right) \delta 7.03(\mathrm{~d}, J=8.6$ Hz, 1H), $6.77-6.70$ (m, 3H), 5.54 (s, 1H), 3.93 (s, 3H), 3.91 (s, 6H), 3.90 (s, 3H), 3.67 $(\mathrm{s}, 3 \mathrm{H}) ;{ }^{13} \mathrm{C} \mathrm{NMR}\left(100 \mathrm{MHz}, \mathrm{CDCl}_{3}\right) \delta 153.1,151.4,146.9,142.7,134.0,129.5,128.8$, 124.7, 107.6, 106.1, 61.2, 61.0, 56.5, 56.2; HRMS (ESI): $\mathrm{m} / \mathrm{z}$ calcd for $\mathrm{C}_{17} \mathrm{H}_{21} \mathrm{O}_{6}$ $[\mathrm{M}+\mathrm{H}]^{+}$321.1288, found 321.1333. IR ( $\mathrm{KBr}$ pellet): 3390(s), 2930 (s), 1490(s), $1220(\mathrm{~s}), 1110(\mathrm{~s}) \mathrm{cm}^{-1}$. 


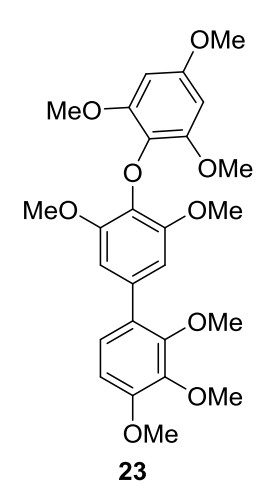

Compound 23: To a mixture of 22 (22 mg, $0.07 \mathrm{mmol}), 1,3,5-$ trimethoxybenzene (2a, $35 \mathrm{mg}, 0.21 \mathrm{mmol})$ and $\mathrm{FeCl}_{3}(2 \mathrm{mg}, 15 \mathrm{~mol}$ $\%)$ in HFIP $(0.14 \mathrm{~mL}, 0.5 \mathrm{M})$, di-t-butylperoxide $(0.039 \mathrm{~mL}, 0.21$ mmol) was added drop-wise at room temperature, stirring was continued for $3 \mathrm{~h}$ and the volatiles were removed under reduced pressure. The crude residue was purified by column chromatography; hexane/ethyl acetate 4:1 to afford compound 23 (23 $\mathrm{mg}, 71 \%$ yield) as

a white solid.

Characterization data of compound 23: ${ }^{1} \mathrm{H}$ NMR $\left(400 \mathrm{MHz}, \mathrm{CDCl}_{3}\right) \delta 7.05(\mathrm{~d}, J=8.6$ $\mathrm{Hz}, 1 \mathrm{H}), 6.73$ (s, 2H), 6.71 (s, 1H), 6.14 (s, 2H), 3.92 (s, 3H), 3.88 (s, 3H), 3.77 (s, 3H), $3.73(\mathrm{~s}, 6 \mathrm{H}), 3.72$ (s, 6H), 3.61 (s, 3H). ${ }^{13} \mathrm{C} \mathrm{NMR}\left(100 \mathrm{MHz}, \mathrm{CDCl}_{3}\right) \delta$ 155.5, 153.0, 152.3, 151.4, 142.7, 136.9, 132.3, 128.8, 124.7, 107.5, 107.4, 92.4, 61.3, 60.9, 56.9, 56.7, 56.2, 55.6; HRMS (ESI): $\mathrm{m} / z$ calcd for $\mathrm{C}_{26} \mathrm{H}_{31} \mathrm{O}_{9}[\mathrm{M}+\mathrm{H}]^{+} 487.1963$, found 487.1942 .

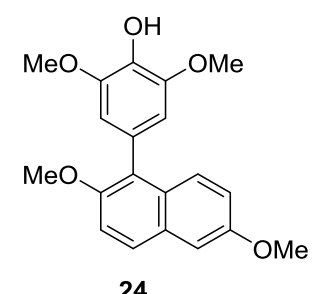

24

Compound 24: To a mixture of 2,6-dimethoxyphenol (1d, $39 \mathrm{mg}$, $0.25 \mathrm{mmol}), 2,6$-dimethoxynaphthalene (2f, $141 \mathrm{mg}, 0.75 \mathrm{mmol})$ and $\mathrm{FeCl}_{3}(6 \mathrm{mg}, 15 \mathrm{~mol} \%)$ in HFIP $(0.5 \mathrm{~mL}, 0.5 \mathrm{M})$, di- $t$ butylperoxide $(0.138 \mathrm{~mL}, 0.75 \mathrm{mmol})$ was added drop-wise at room temperature, stirring was continued for $3 \mathrm{~h}$ and the volatiles were removed under reduced pressure. The crude residue was purified by column chromatography; hexane/ethyl acetate 10:1 to afford compound $\mathbf{2 4}$ (39 $\mathrm{mg}$, $44 \%$ yield) as a yellow solid.

Characterization data of compound 24: ${ }^{1} \mathrm{H}$ NMR $\left(400 \mathrm{MHz}, \mathrm{CDCl}_{3}\right) \delta 7.76(\mathrm{~d}, \mathrm{~J}=9.0$ $\mathrm{Hz}, 1 \mathrm{H}), 7.47(\mathrm{~d}, \mathrm{~J}=9.3 \mathrm{~Hz}, 1 \mathrm{H}), 7.34(\mathrm{~d}, \mathrm{~J}=9.0 \mathrm{~Hz}, 1 \mathrm{H}), 7.14(\mathrm{~d}, \mathrm{~J}=2.5 \mathrm{~Hz}, 1 \mathrm{H})$, $7.05(\mathrm{dd}, \mathrm{J}=9.3,2.6 \mathrm{~Hz}, 1 \mathrm{H}), 6.59$ (s, 2H), 5.62 (s, 1H), 3.92 (s, 3H), 3.88 (s, 6H), 3.84 $(\mathrm{s}, 3 \mathrm{H}) ;{ }^{13} \mathrm{C} \mathrm{NMR}\left(100 \mathrm{MHz}, \mathrm{CDCl}_{3}\right) \delta 156.2,152.5,147.0,133.9,130.1,129.4,127.6$, 127.5, 127.1, 126.0, 119.3, 114.7, 107.7, 105.8, 57.2, 56.4, 55.4; HRMS (ESI): $\mathrm{m} / \mathrm{z}$ calcd for $\mathrm{C}_{20} \mathrm{H}_{20} \mathrm{O}_{5} \mathrm{Na}[\mathrm{M}+\mathrm{Na}]^{+}$363.1203, found 363.1189. IR (KBr pellet): 3150(s), $2910(\mathrm{~m}), 15900(\mathrm{~s}), 1260(\mathrm{~s}), 1110(\mathrm{~s}) \mathrm{cm}^{-1}$. 


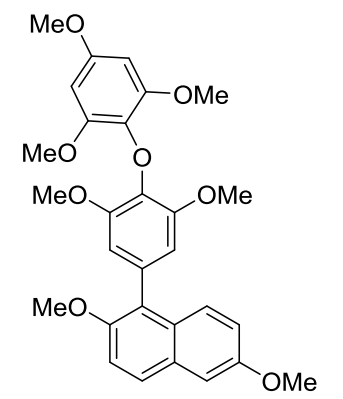

25

Compound 25: To a mixture of 24 (85 mg, $0.25 \mathrm{mmol}), 1,3,5-$ trimethoxybenzene (2a, $126 \mathrm{mg}, 0.75 \mathrm{mmol})$ and $\mathrm{FeCl}_{3}(6 \mathrm{mg}, 15$ mol \%) in HFIP $(0.5 \mathrm{~mL}, 0.5 \mathrm{M})$, di-t-butylperoxide $(0.138 \mathrm{~mL}$, $0.75 \mathrm{mmol}$ ) was added drop-wise at room temperature, stirring was continued for $3 \mathrm{~h}$ and the volatiles were removed under reduced pressure. The crude residue was purified by column chromatography; hexane/ethyl acetate 7:3 to afford compound 25

(94 mg, 76\% yield) as a yellow solid.

Characterization data of compound 25: ${ }^{1} \mathrm{H}$ NMR $\left(400 \mathrm{MHz}, \mathrm{CDCl}_{3}\right) \delta 7.74(\mathrm{~d}, J=9.0$ $\mathrm{Hz}, 1 \mathrm{H}), 7.48$ (d, $J=9.3 \mathrm{~Hz}, 1 \mathrm{H}), 7.32$ (d, $J=9.0 \mathrm{~Hz}, 1 \mathrm{H}), 7.13$ (d, $J=2.6 \mathrm{~Hz}, 1 \mathrm{H})$, $7.04(\mathrm{dd}, J=9.3,2.6 \mathrm{~Hz}, 1 \mathrm{H}), 6.56(\mathrm{~s}, 2 \mathrm{H}), 6.18$ (s, 2H), 3.90 (s, 3H), 3.79 (s, 3H), 3.78 (overlapped, 9H), $3.71(\mathrm{~s}, 6 \mathrm{H}) ;{ }^{13} \mathrm{C} \mathrm{NMR}\left(100 \mathrm{MHz}, \mathrm{CDCl}_{3}\right) \delta 156.2,155.5,152.5$, $152.3,151.5,136.8,132.3,130.5,130.2,129.2$, 127.6, 127.1, 126.5, 119.1, 115.4, 109.0, 105.8, 92.6, 57.5, 56.8, 56.7, 55.6, 55.4; HRMS (ESI): $m / z$ calcd for $\mathrm{C}_{29} \mathrm{H}_{30} \mathrm{O}_{8} \mathrm{Na}$ $[\mathrm{M}+\mathrm{Na}]^{+}$529.1833, found 529.1817 .

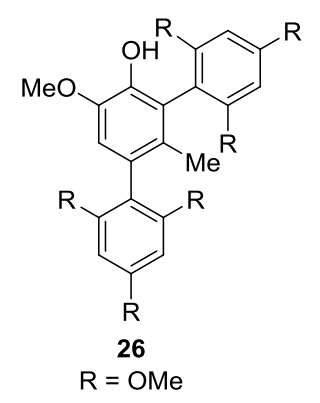

Compound 26: To a mixture of 2-methoxy-5-methyl phenol (35 $\mathrm{mg}, 0.25 \mathrm{mmol}), 1,3,5$-trimethoxybenzene (2a, $210 \mathrm{mg}, 1.25 \mathrm{mmol})$ and $\mathrm{FeCl}_{3}(6 \mathrm{mg}, 15 \mathrm{~mol} \%)$ in $\operatorname{HFIP}(0.5 \mathrm{~mL}, 0.5 \mathrm{M})$, di- $t$ butylperoxide $(0.230 \mathrm{~mL}, 1.25 \mathrm{mmol})$ was added drop-wise at room temperature, stirring was continued for $6 \mathrm{~h}$ and the volatiles were removed under reduced pressure. The crude residue was purified by column chromatography; hexane/ethyl acetate 4:6 to afford compound $\mathbf{2 6}$ (49 mg, $42 \%$ yield) as a white solid.

Characterization data of compound 26: ${ }^{1} \mathrm{H}$ NMR (400 MHz, $\left.\mathrm{CDCl}_{3}\right) \delta 6.69(\mathrm{~s}, 1 \mathrm{H})$, $6.27(\mathrm{~s}, 2 \mathrm{H}), 6.24(\mathrm{~s}, 2 \mathrm{H}), 5.39$ (s, 1H), 3.87 (overlapped, 6H), 3.85 (s, 3H), 3.74 (s, 6H), 3.73 (s, 6H), 1.68 (s, 3H); ${ }^{13} \mathrm{C}$ NMR (100 MHz, $\left.\mathrm{CDCl}_{3}\right) \delta 161.1,160.3,158.9$, 158.7, 143.8, 142.3, 130.9, 124.3, 120.4, 113.0, 112.8, 107.1, 91.4, 91.1, 56.1, 56.0, 55.6, $55.4(\times 2), 16.6$; HRMS (ESI): $\mathrm{m} / z$ calcd for $\mathrm{C}_{26} \mathrm{H}_{31} \mathrm{O}_{8}[\mathrm{M}+\mathrm{H}]^{+} 471.2013$, found 471.2015 . 


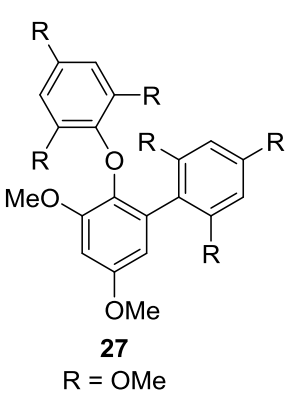

Compound 27: To a mixture of 2,4-dimethoxyphenol (39 mg, 0.25 mmol), 1,3,5-trimethoxybenzene (2a, $210 \mathrm{mg}, 1.25 \mathrm{mmol})$ and $\mathrm{FeCl}_{3}(6 \mathrm{mg}, 15 \mathrm{~mol} \%)$ in HFIP $(0.5 \mathrm{~mL}, 0.5 \mathrm{M})$, di-t-butylperoxide $(0.230 \mathrm{~mL}, 1.25 \mathrm{mmol})$ was added drop-wise at room temperature, stirring was continued for $3 \mathrm{~h}$ and the volatiles were removed under reduced pressure. The crude residue was purified by column chromatography; hexane/ethyl acetate $6: 4$ to afford compound 27 (58 mg, $48 \%$ yield) as a white solid.

Characterization data of compound 27: ${ }^{1} \mathrm{H}$ NMR $\left(500 \mathrm{MHz}, \mathrm{CDCl}_{3}\right) \delta 6.51(\mathrm{~d}, J=2.6$ $\mathrm{Hz}, 1 \mathrm{H}), 6.10$ (s, 1H), 5.89 (s, 2H), 5.80 (s, 2H), 3.88 (s, 3H), 3.77 (s, 3H), 3.74 (s, 3H), $3.67(\mathrm{~s}, 3 \mathrm{H}), 3.54(\mathrm{~s}, 6 \mathrm{H}), 3.48(\mathrm{~s}, 6 \mathrm{H}) ;{ }^{13} \mathrm{C} \mathrm{NMR}\left(125 \mathrm{MHz}, \mathrm{CDCl}_{3}\right) \delta 160.6,158.5$, 155.1, 154.6, 152.4, 152.0, 142.0, 125.6, 108.6, 107.2, 99.2, 91.7, 89.7, 56.2, 56.1, 55.7, 55.5, 55.5, 55.4; HRMS (ESI): $m / z$ calcd for $\mathrm{C}_{26} \mathrm{H}_{30} \mathrm{O} 9 \mathrm{Na}[\mathrm{M}+\mathrm{Na}]^{+} 509.1782$, found 509.1781.

Compound 28a and 28b: To a mixture of 2-methoxy-4-methylphenol (35 mg, 0.25 mmol), 5-methoxy-1,3-benzodioxole (2d, $114 \mathrm{mg}, 0.75 \mathrm{mmol})$ and $\mathrm{FeCl}_{3}(6 \mathrm{mg}, 15$ mol \%) in HFIP $(0.5 \mathrm{~mL}, 0.5 \mathrm{M})$, di-t-butylperoxide $(0.138 \mathrm{~mL}, 0.75 \mathrm{mmol})$ was added drop-wise at room temperature, stirring was continued for $3.5 \mathrm{~h}$ and the volatiles were removed under reduced pressure. The crude residue was purified by column chromatography; hexane/ethyl acetate 9:1 to afford compound 28a (44 mg, 61\% yield) as a yellow liquid and hexane/ethyl acetate 17:2 compound $\mathbf{2 8 b}$ (32 $\mathrm{mg}, 29 \%$ yield) as a white solid.

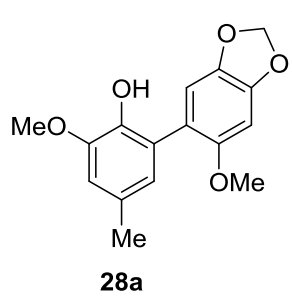

Characterization data of compound 28a: ${ }^{1} \mathrm{H}$ NMR $(400 \mathrm{MHz}$, $\left.\mathrm{CDCl}_{3}\right) \delta 6.79(\mathrm{~s}, 1 \mathrm{H}), 6.69(\mathrm{~s}, 1 \mathrm{H}), 6.64(\mathrm{~s}, 2 \mathrm{H}), 5.96(\mathrm{~s}, 2 \mathrm{H}), 5.84$ (s, 1H), $3.90(\mathrm{~s}, 3 \mathrm{H}), 3.76(\mathrm{~s}, 3 \mathrm{H}), 2.31(\mathrm{~s}, 3 \mathrm{H}) ;{ }^{13} \mathrm{C} \mathrm{NMR}(100 \mathrm{MHz}$, $\left.\mathrm{CDCl}_{3}\right) \delta 151.6,148.0,147.4,141.8,141.0,129.2,125.2,123.7$, 119.4, 111.3, 111.2, 101.5, 95.6, 57.3, 56.1, 21.3; HRMS (ESI): $\mathrm{m} / \mathrm{z}$ calcd for $\mathrm{C}_{16} \mathrm{H}_{17} \mathrm{O}_{5}[\mathrm{M}+\mathrm{H}]^{+}$289.1026, found 289.1071. 

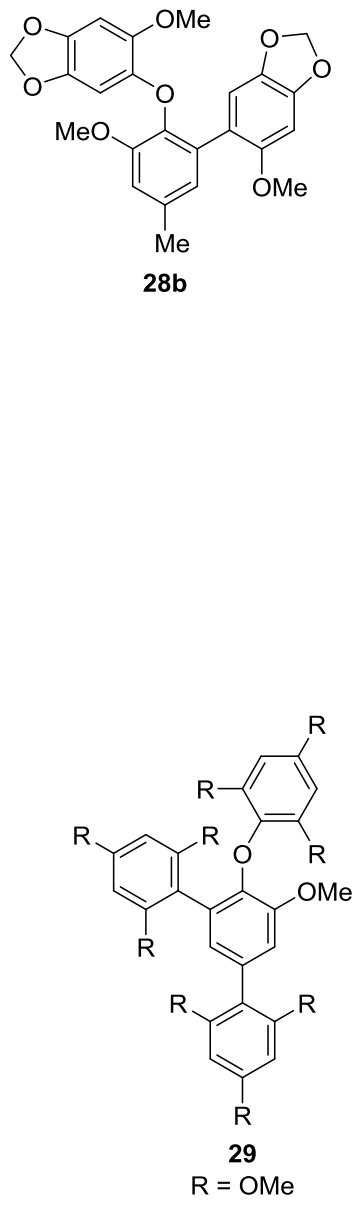

Characterization data of compound 28b: ${ }^{1} \mathrm{H}$ NMR $(400 \mathrm{MHz}$, $\left.\mathrm{CDCl}_{3}\right) \delta 6.75(\mathrm{~d}, J=1.9 \mathrm{~Hz}, 1 \mathrm{H}), 6.70(\mathrm{dd}, J=1.9,0.6 \mathrm{~Hz}, 1 \mathrm{H})$, $6.67(\mathrm{~s}, 1 \mathrm{H}), 6.46(\mathrm{~s}, 1 \mathrm{H}), 6.45(\mathrm{~s}, 1 \mathrm{H}), 6.16(\mathrm{~s}, 1 \mathrm{H}), 5.88(\mathrm{~s}, 2 \mathrm{H})$, 5.78 (s, 2H), 3.76 (s, 3H), 3.69 (s, 3H), 3.60 (s, 3H), 2.37 (s, 3H); ${ }^{13} \mathrm{C}$ NMR $\left(100 \mathrm{MHz}, \mathrm{CDCl}_{3}\right) \delta 152.2,147.5,143.5,143.1,140.9$, 140.8, 140.6, 139.9, 134.7, 133.1, 127.8, 124.3, 118.9, 112.7, 111.0, 101.2, 101.0, 97.9, 97.4, 94.6, 77.2, 58.0, 56.5, 56.1, 21.6; HRMS (ESI): $m / z$ calcd for $\mathrm{C}_{24} \mathrm{H}_{22} \mathrm{O}_{8} \mathrm{Na}[\mathrm{M}+\mathrm{Na}]^{+}$461.1207, found 461.1195 .

Compound 29: To a mixture of 2-methoxyphenol (31 mg, 0.25 mmol), 1,3,5-trimethoxybenzene (2a, $210 \mathrm{mg}, 1.25 \mathrm{mmol})$ and $\mathrm{FeCl}_{3}(6 \mathrm{mg}, 15 \mathrm{~mol} \%)$ in $\mathrm{HFIP}(0.5 \mathrm{~mL}, 0.5 \mathrm{M})$, di-t-butylperoxide $(0.230 \mathrm{~mL}, 1.25 \mathrm{mmol})$ was added drop-wise at room temperature, stirring was continued at $40{ }^{\circ} \mathrm{C}$ for $20 \mathrm{~h}$ and the volatiles were removed under reduced pressure. The crude residue was purified by column chromatography; hexane/ethyl acetate $4: 6$ to afford compound 29 (52 mg, 33\% yield) as a white solid.

Characterization data of compound 29: ${ }^{1} \mathrm{H}$ NMR $\left(500 \mathrm{MHz}, \mathrm{CDCl}_{3}\right) \delta 6.91(\mathrm{~d}, J=2.1$ $\mathrm{Hz}, 1 \mathrm{H}), 6.60(\mathrm{~d}, J=2.1 \mathrm{~Hz}, 1 \mathrm{H}), 6.20(\mathrm{~s}, 2 \mathrm{H}), 5.89(\mathrm{~s}, 2 \mathrm{H}), 5.81(\mathrm{~s}, 2 \mathrm{H}), 3.88(\mathrm{~s}, 3 \mathrm{H})$, $3.83(\mathrm{~s}, 3 \mathrm{H}), 3.77(\mathrm{~s}, 3 \mathrm{H}), 3.69(\mathrm{~s}, 6 \mathrm{H}), 3.67(\mathrm{~s}, 3 \mathrm{H}), 3.55(\mathrm{~s}, 6 \mathrm{H}), 3.48(\mathrm{~s}, 6 \mathrm{H}) ;{ }^{13} \mathrm{C}$ NMR $\left(100 \mathrm{MHz}, \mathrm{CDCl}_{3}\right) \delta 160.4,160.2,158.6,155.1,152.0,151.1,127.7,127.4$, 124.2, 114.5, 113.6, 92.0, 91.9, 89.9, 56.4, 56.2, 56.0, 55.7, 55.5; HRMS (ESI): $\mathrm{m} / \mathrm{z}$ calcd for $\mathrm{C}_{34} \mathrm{H}_{38} \mathrm{O}_{11} \mathrm{Na}[\mathrm{M}+\mathrm{Na}]^{+}$645.2306, found 645.2312 . 


\section{Spectral $\left({ }^{1} \mathrm{H} \&{ }^{13} \mathrm{C}\right)$ data:}

${ }^{1} \mathrm{H}$ NMR spectrum of compound $\mathbf{1 b}\left(\mathrm{CDCl}_{3}, 400 \mathrm{MHz}\right)$

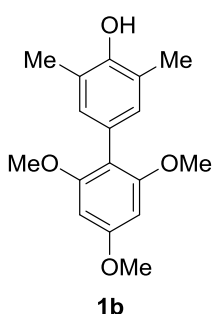

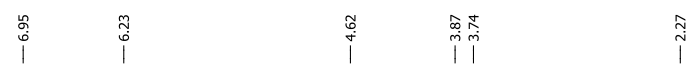

$1 \mathrm{~b}$

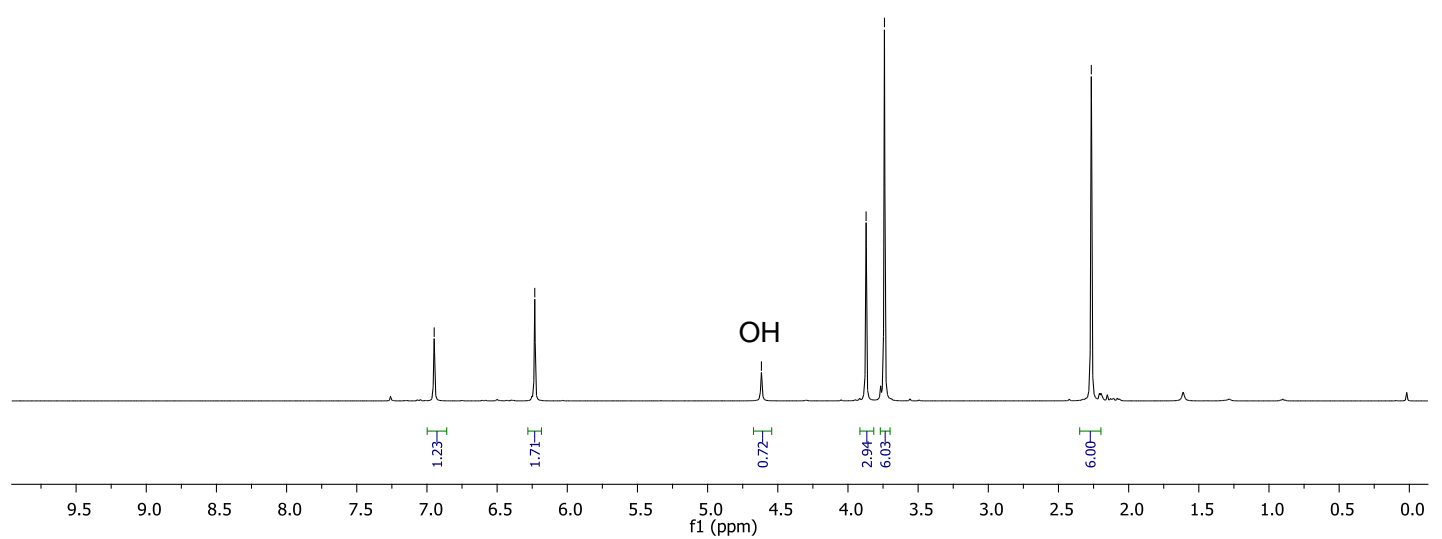

${ }^{13} \mathrm{C}$ NMR spectrum of compound $\mathbf{1 b}\left(\mathrm{CDCl}_{3}, 100 \mathrm{MHz}\right)$

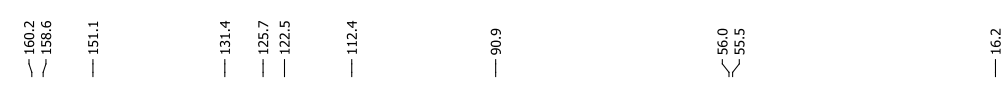
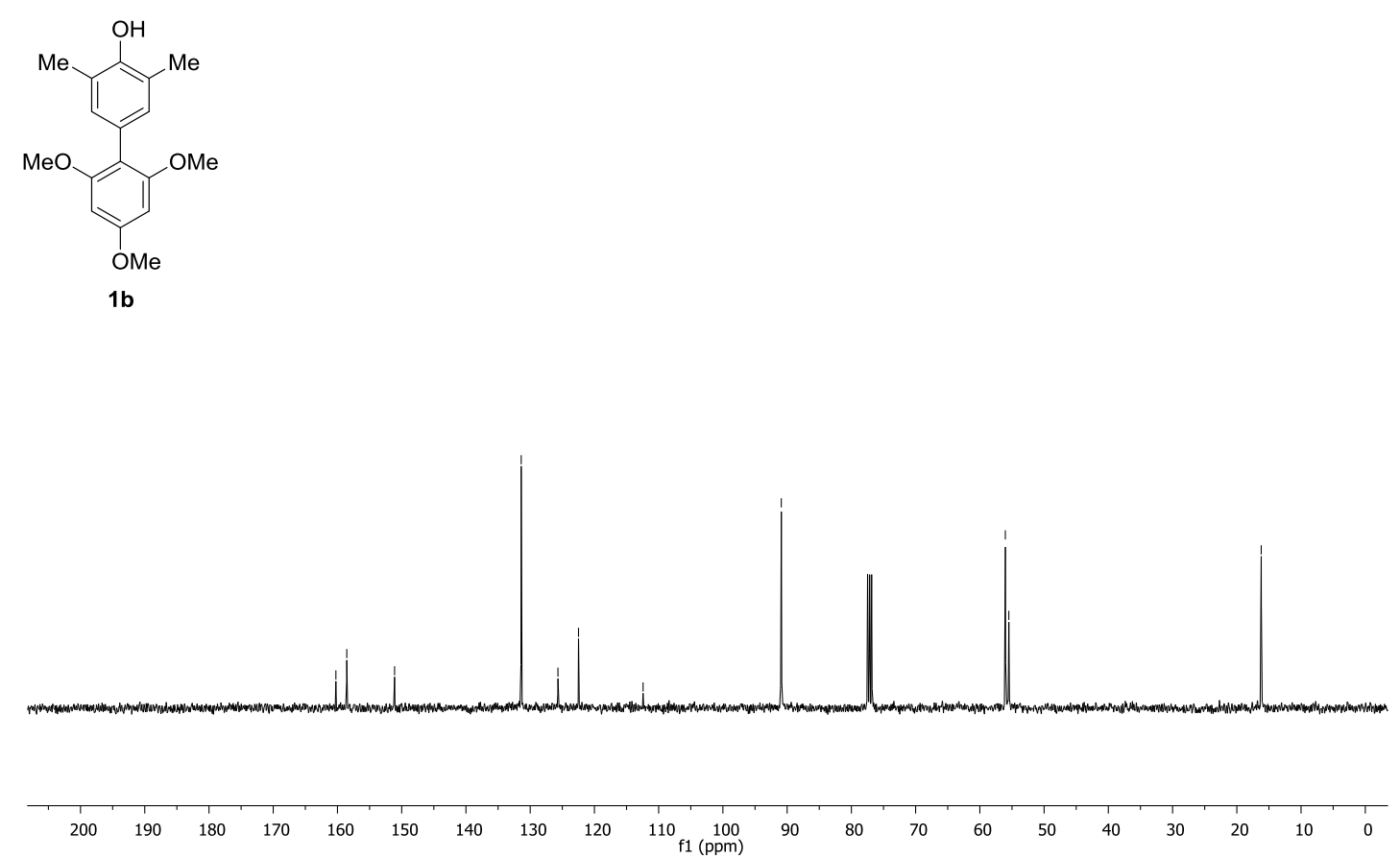
${ }^{1} \mathrm{H}$ NMR spectrum of compound 1ei $\left(\mathrm{CDCl}_{3}, 400 \mathrm{MHz}\right)$

@

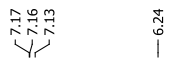

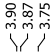<smiles>COc1cc(OC)c(-c2cc(OC)c(O)c(OC)c2)c(OC)c1</smiles>

1 ei

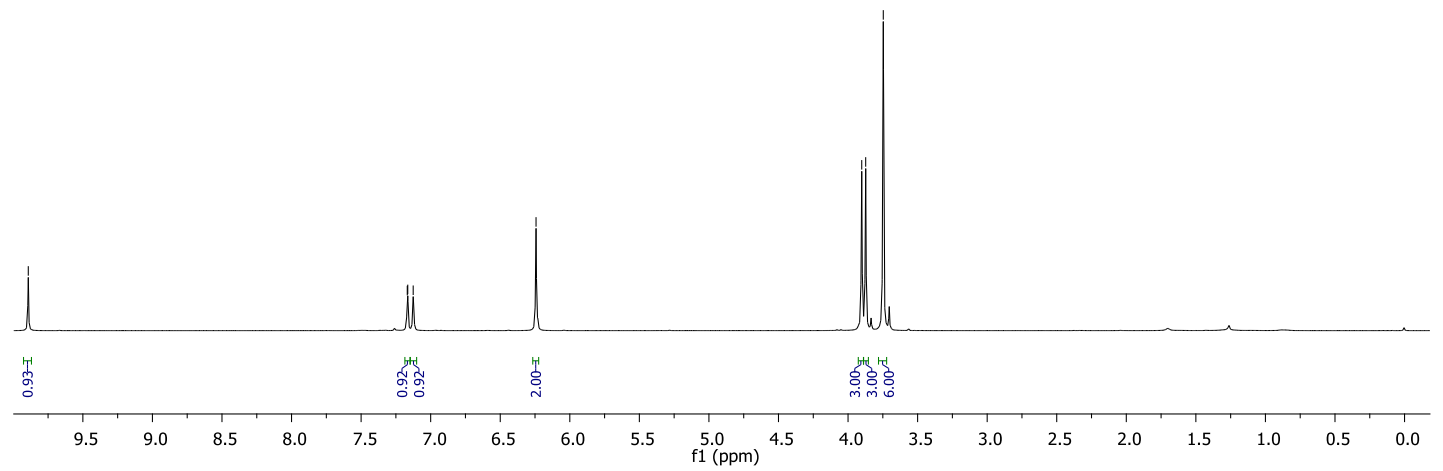

${ }^{13} \mathrm{C}$ NMR spectrum of compound 1 ei $\left(\mathrm{CDCl}_{3}, 100 \mathrm{MHz}\right)$

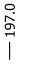

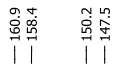

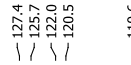
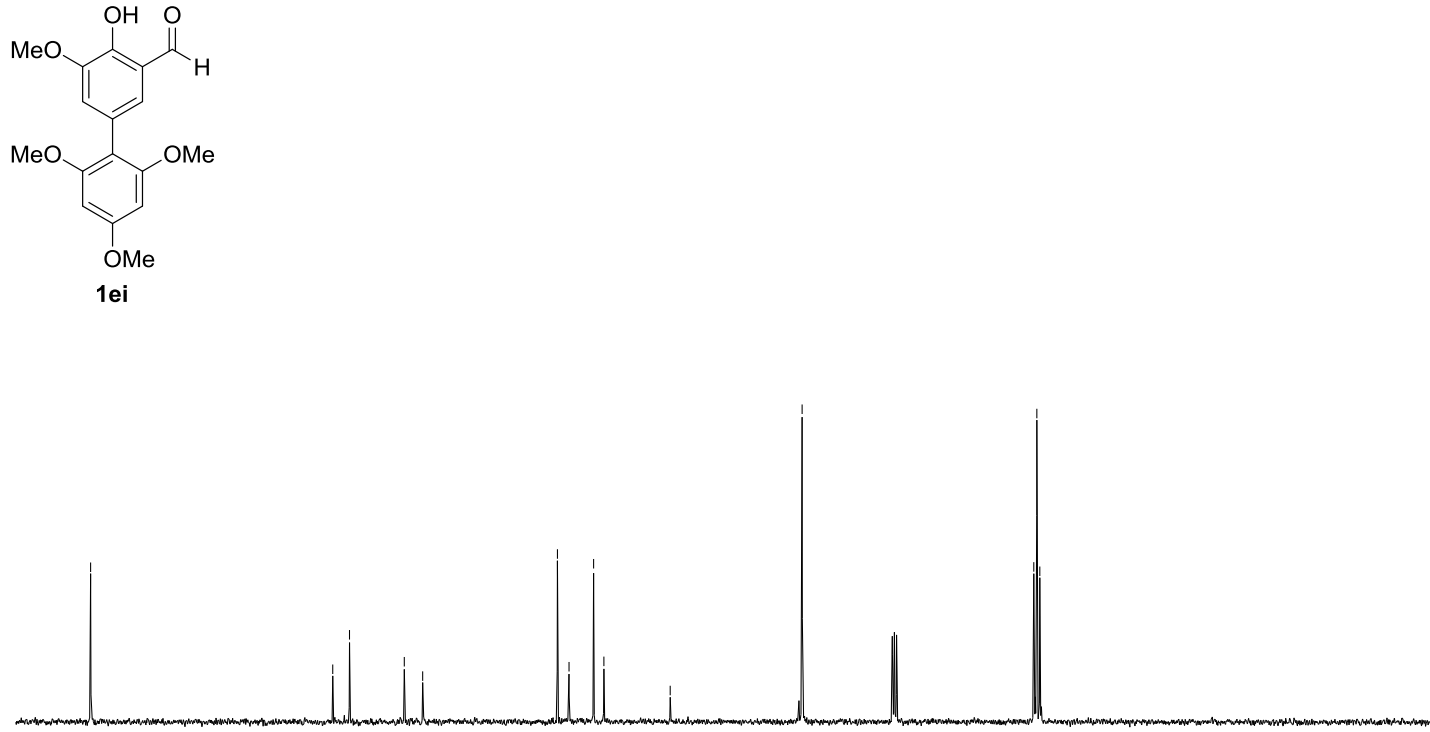

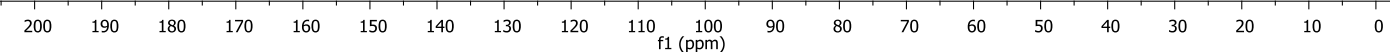


${ }^{1} \mathrm{H}$ NMR spectrum of compound $1 \mathrm{e}\left(\mathrm{CDCl}_{3}, 400 \mathrm{MHz}\right)$

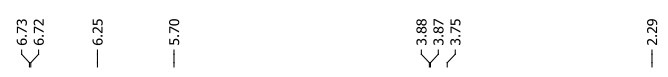<smiles>COc1cc(OC)c(-c2cc(OC)c(O)c(OC)c2)c(OC)c1</smiles>

$1 \mathrm{e}$

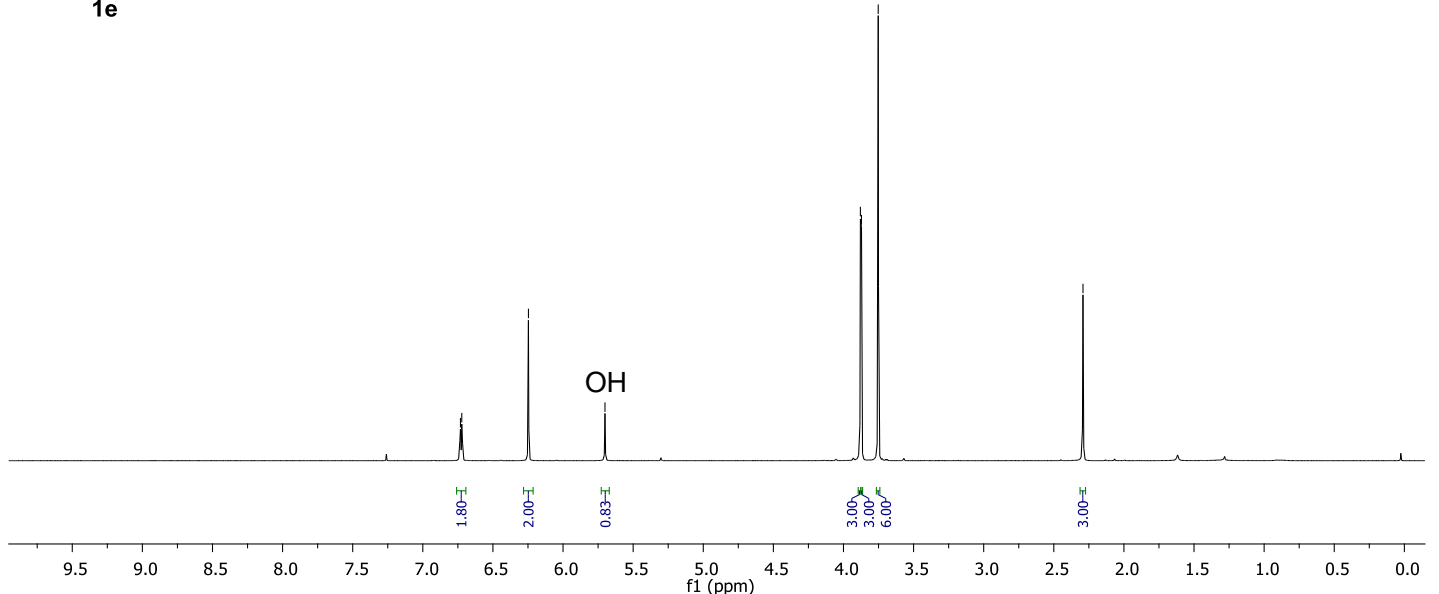

${ }^{13} \mathrm{C}$ NMR spectrum of compound $\mathbf{1 e}\left(\mathrm{CDCl}_{3}, 100 \mathrm{MHz}\right)$
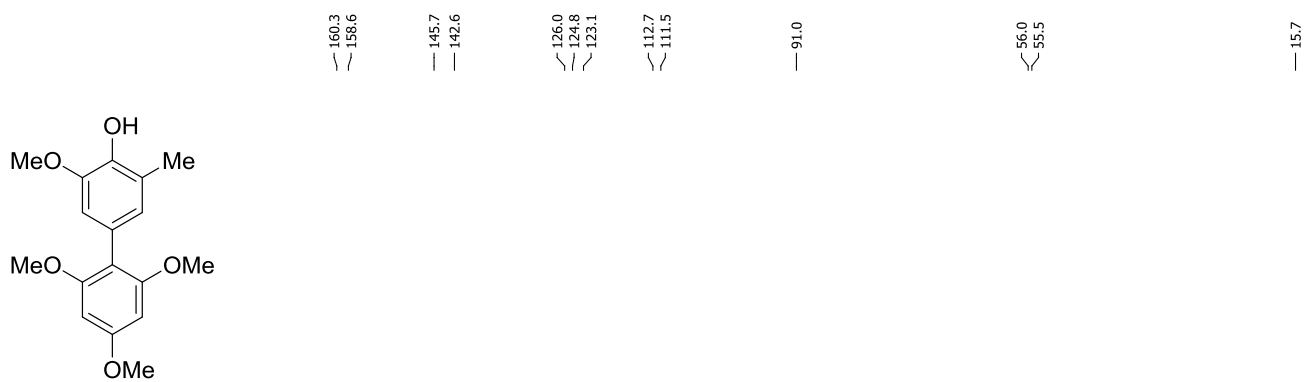

1e

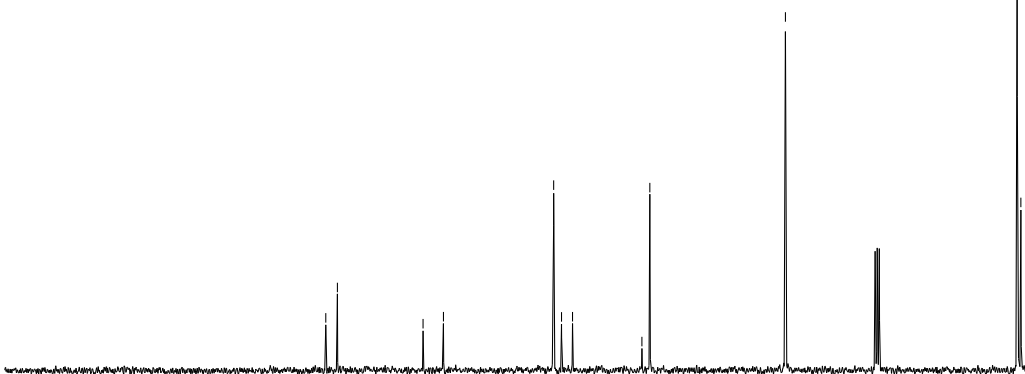

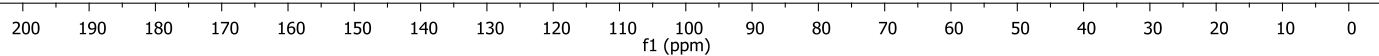


${ }^{1} \mathrm{H}$ NMR spectrum of compound $\mathbf{1 f}\left(\mathrm{CDCl}_{3}, 400 \mathrm{MHz}\right)$

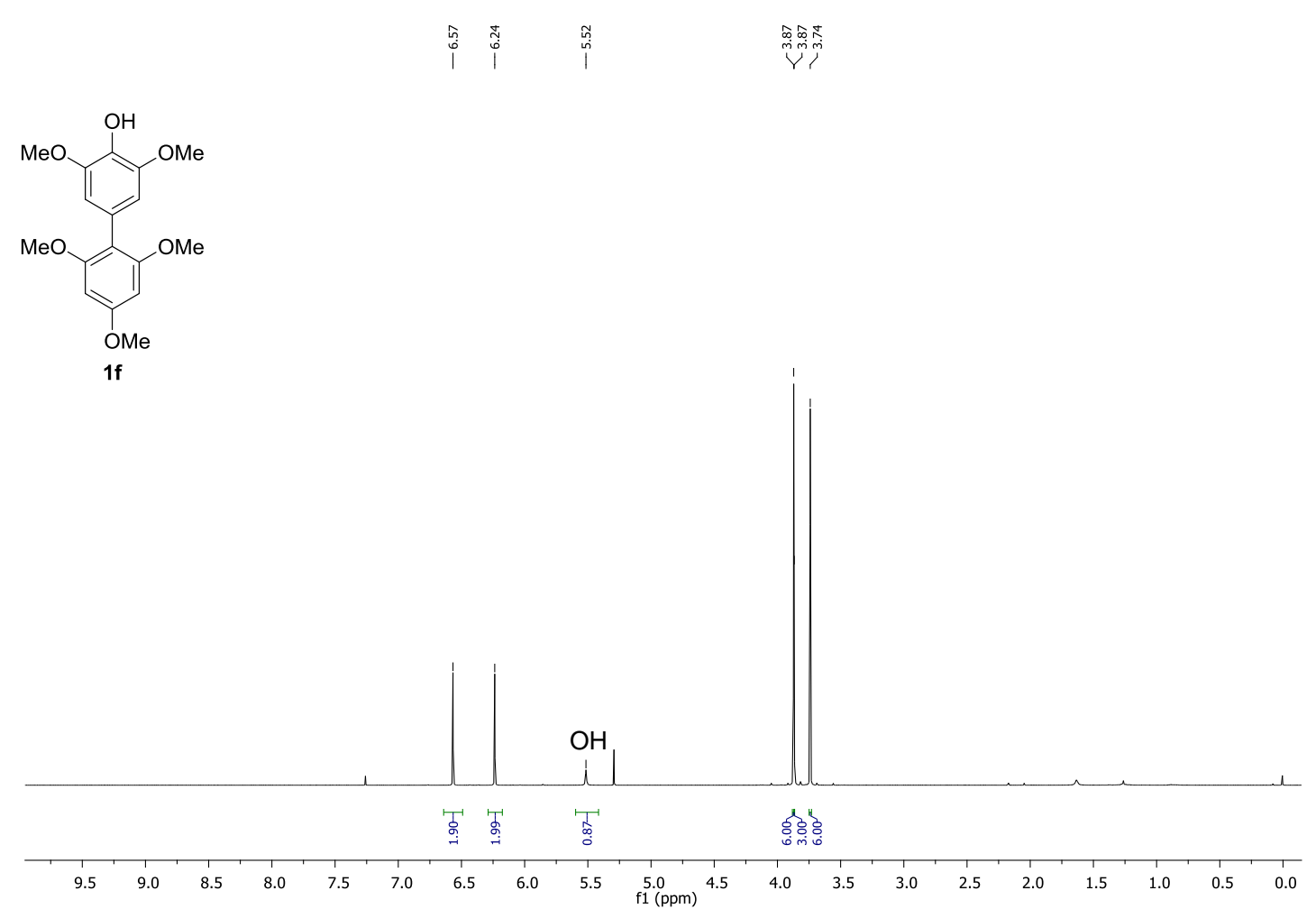

${ }^{13} \mathrm{C}$ NMR spectrum of compound $\mathbf{1 f}\left(\mathrm{CDCl}_{3}, 100 \mathrm{MHz}\right)$
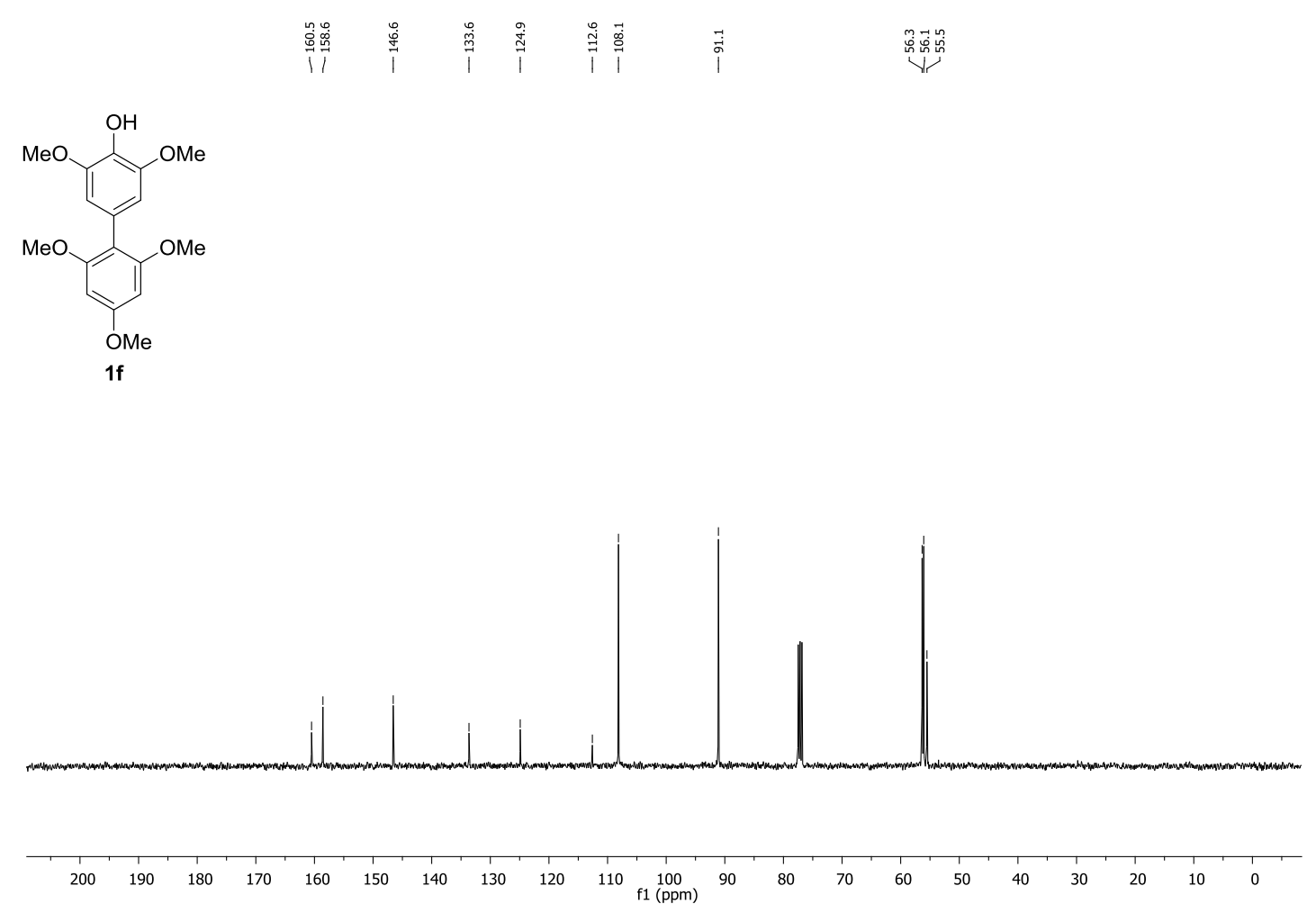
${ }^{1} \mathrm{H}$ NMR spectrum of compound $\mathbf{1 g}\left(\mathrm{CDCl}_{3}, 400 \mathrm{MHz}\right)$

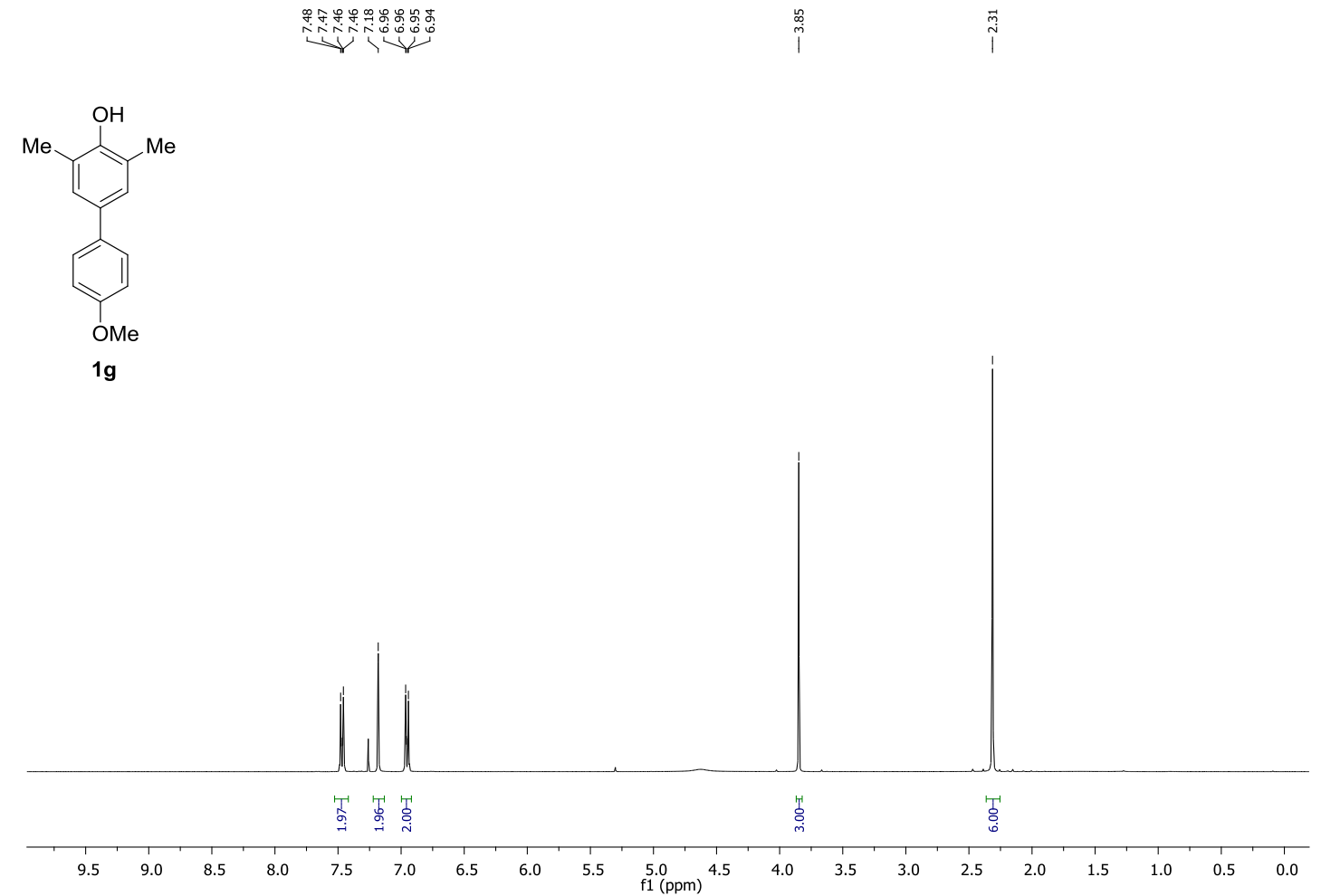

${ }^{13} \mathrm{C}$ NMR spectrum of compound $\mathbf{1 g}\left(\mathrm{CDCl}_{3}, 100 \mathrm{MHz}\right)$
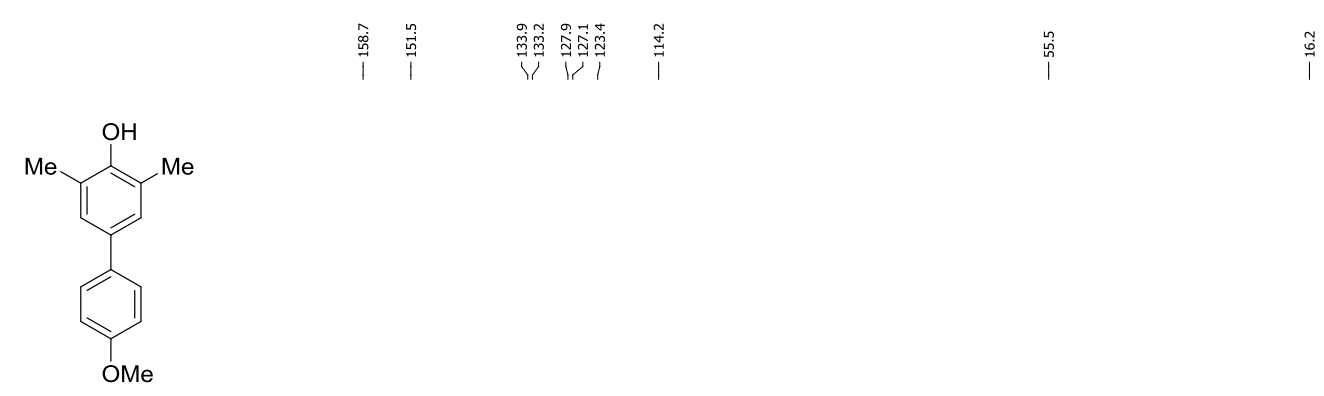

$1 \mathrm{~g}$

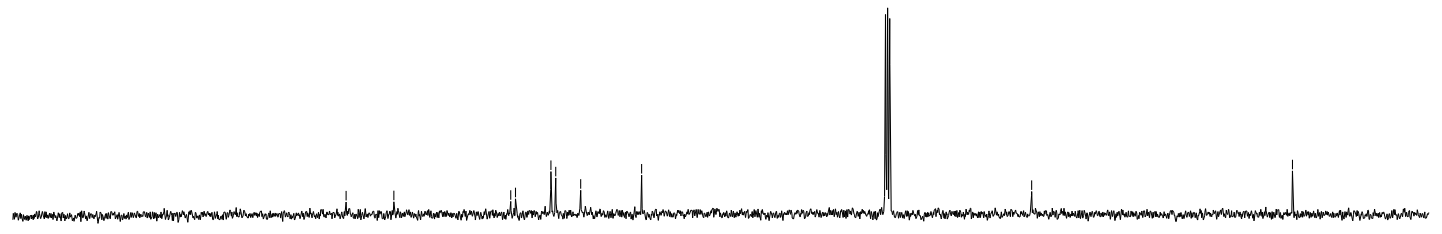

$\begin{array}{llllllllllllllllllllllll}200 & 190 & 180 & 170 & 160 & 150 & 140 & 130 & 120 & 110 & 100 & 90 & 80 & 70 & 60 & 50 & 40 & 30 & 20 & 10 & 0\end{array}$ 
${ }^{1} \mathrm{H}$ NMR spectrum of compound $\mathbf{1 h}\left(\mathrm{CDCl}_{3}, 500 \mathrm{MHz}\right)$
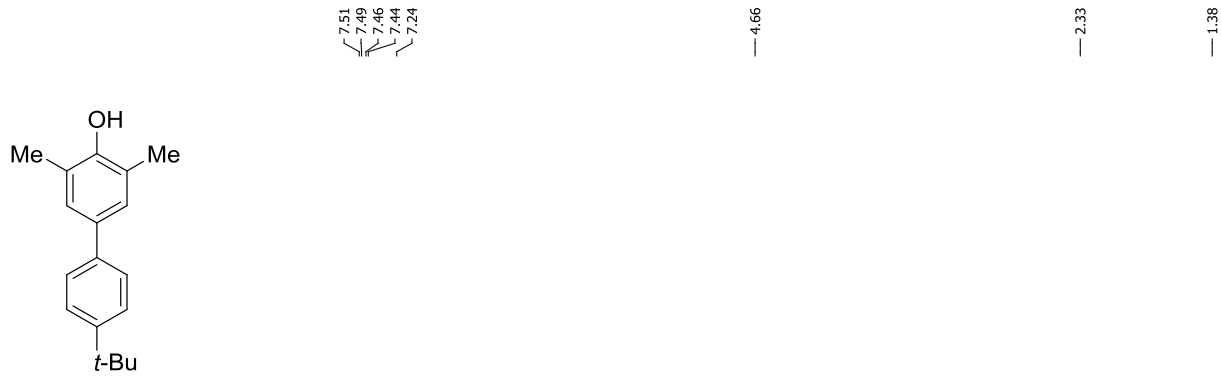

1h

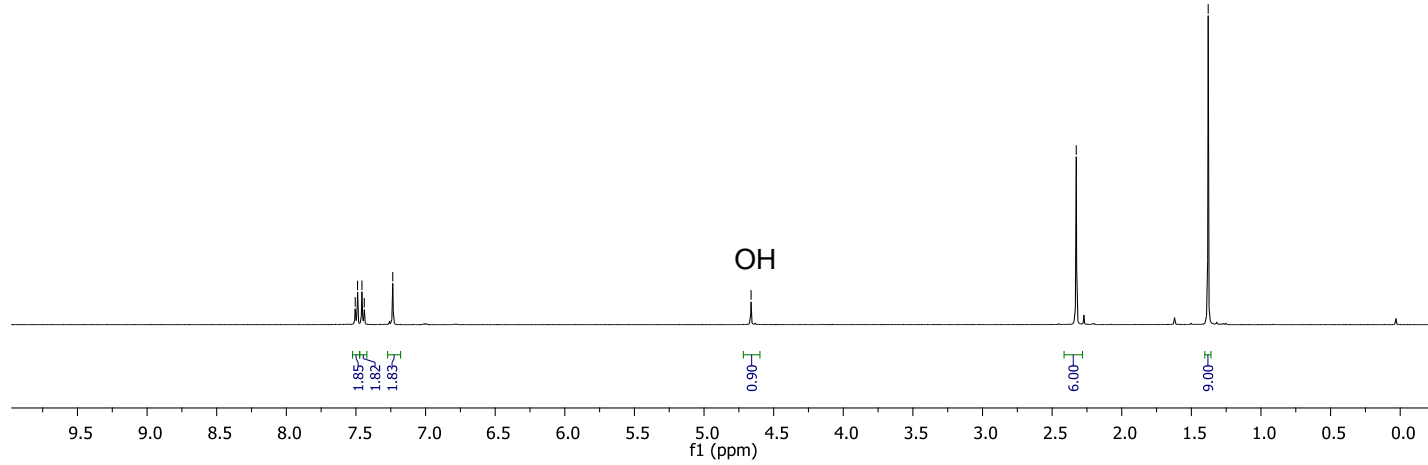

${ }^{13} \mathrm{C}$ NMR spectrum of compound $\mathbf{1 h}\left(\mathrm{CDCl}_{3}, 125 \mathrm{MHz}\right)$
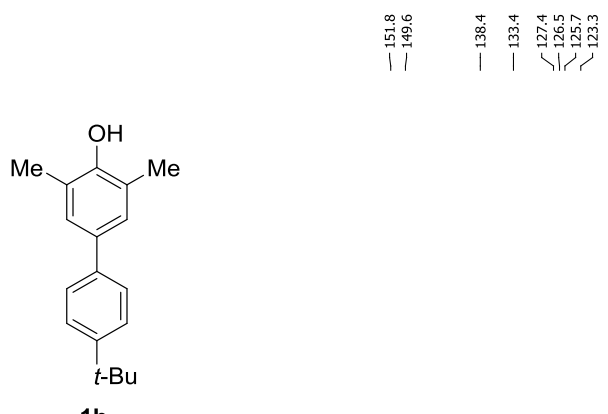

$1 \mathrm{~h}$

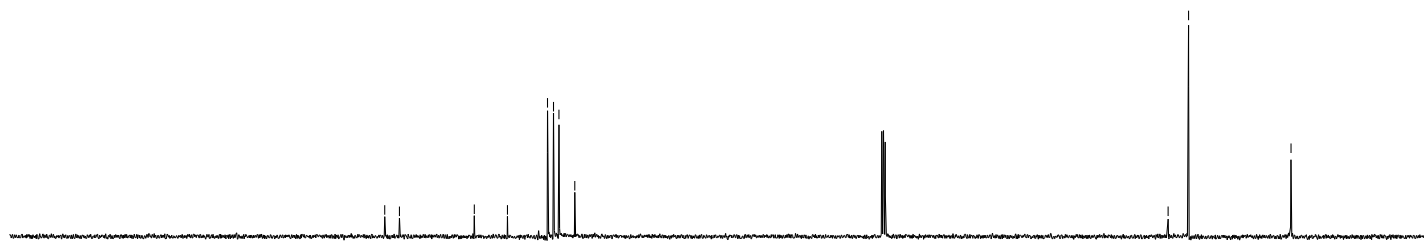

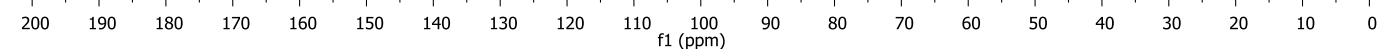


${ }^{1} \mathrm{H}$ NMR spectrum of compound $\mathbf{1 i}\left(\mathrm{CDCl}_{3}, 500 \mathrm{MHz}\right)$<smiles>Cc1cc(-c2ccc(F)cc2)cc(C)c1O</smiles>
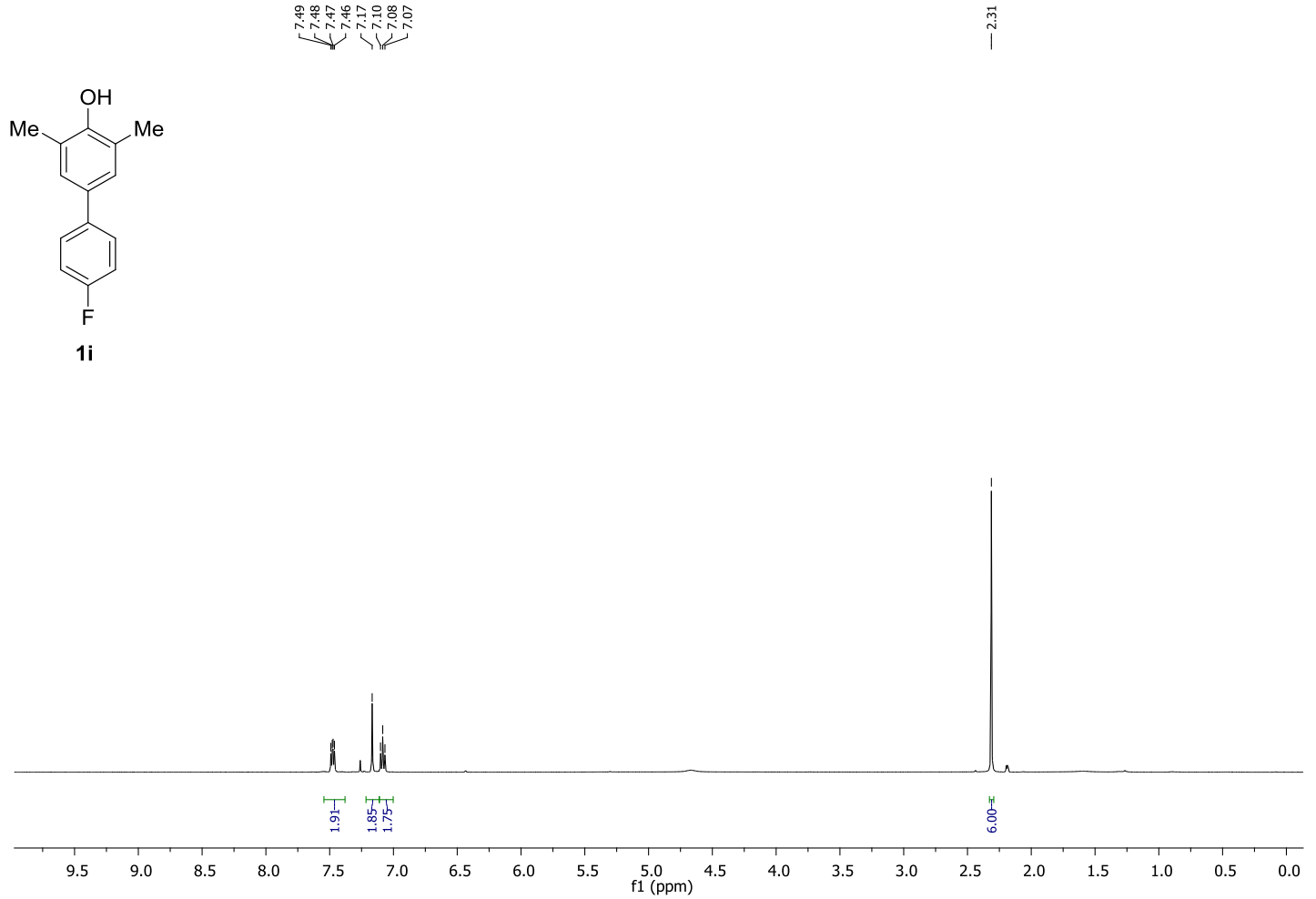

${ }^{13} \mathrm{C}$ NMR spectrum of compound $\mathbf{1 i}\left(\mathrm{CDCl}_{3}, 125 \mathrm{MHz}\right)$

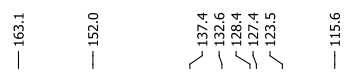
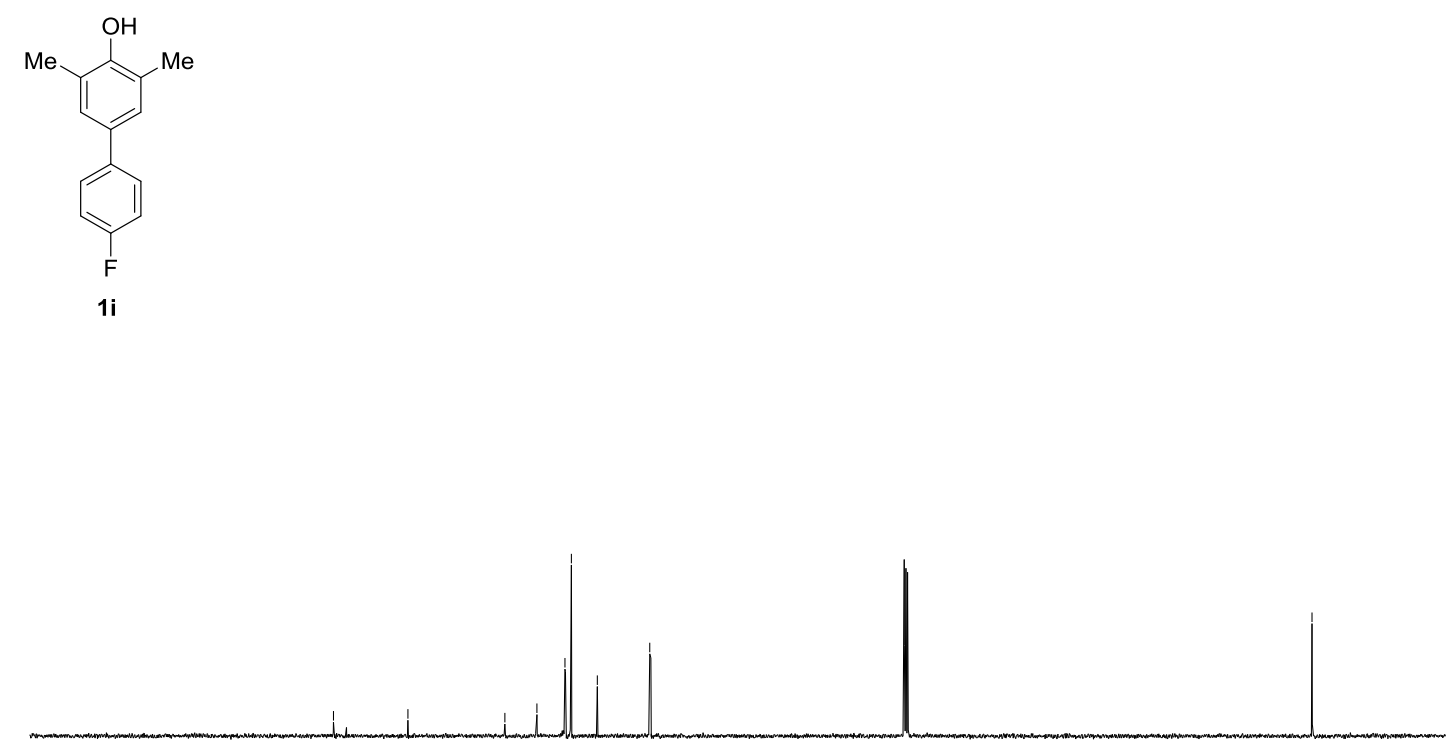

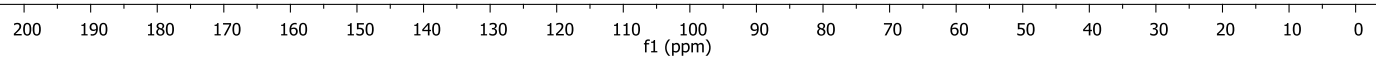


${ }^{19} \mathrm{~F}$ NMR spectrum of compound $\mathbf{1 i}\left(\mathrm{CDCl}_{3}, 377 \mathrm{MHz}\right)$
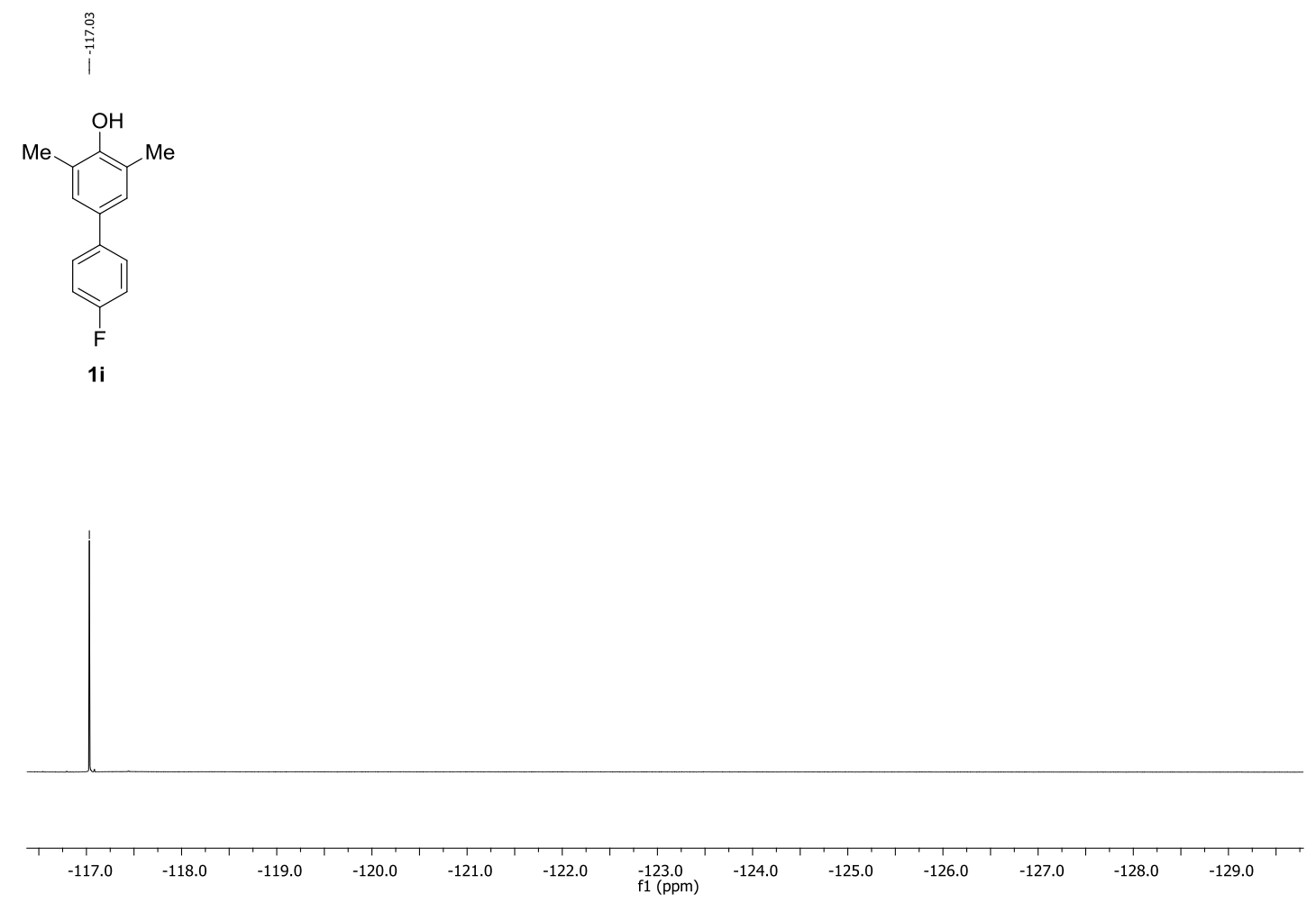
${ }^{1} \mathrm{H}$ NMR spectrum of compound $3\left(\mathrm{CDCl}_{3}, 400 \mathrm{MHz}\right)$

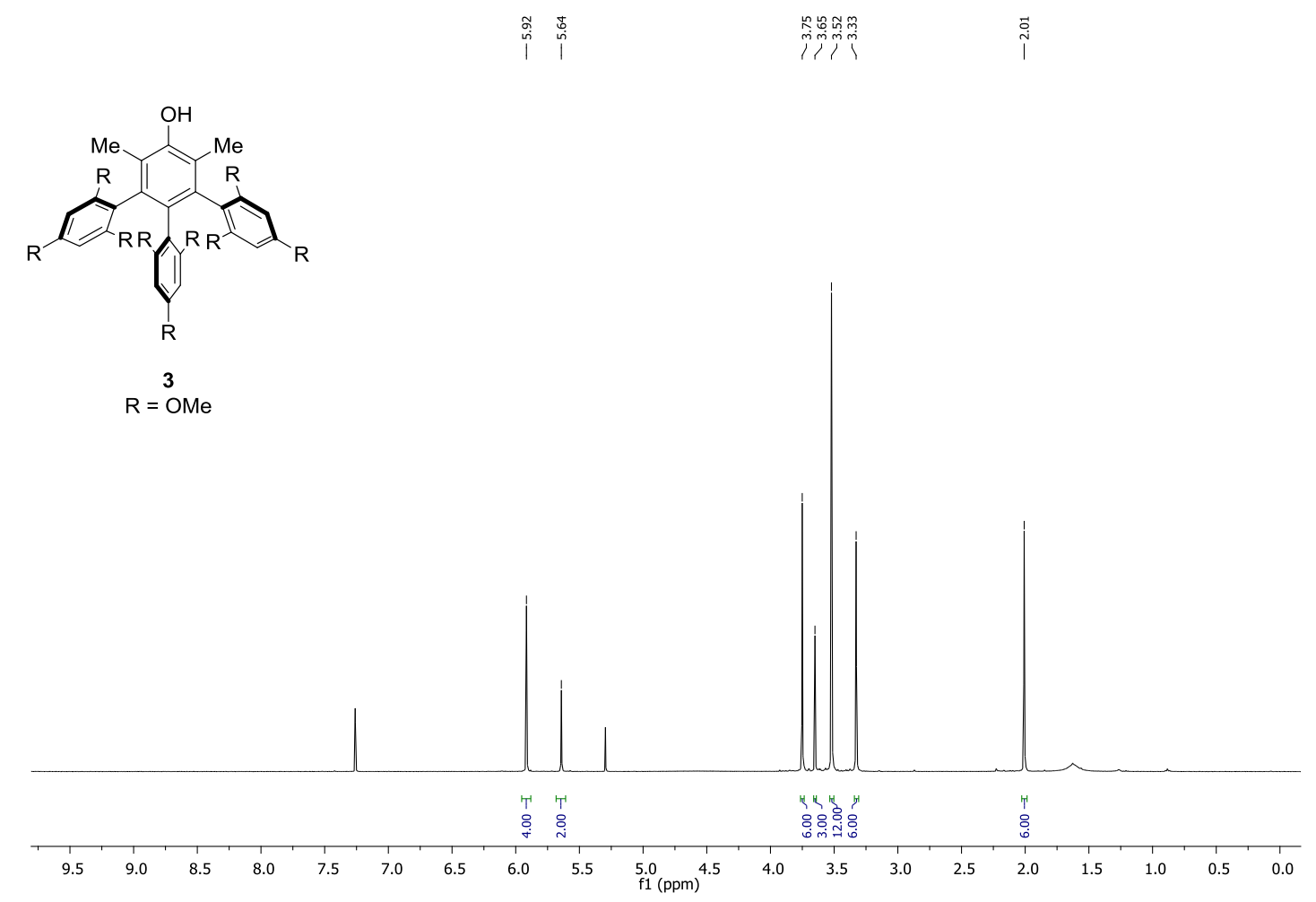

${ }^{13} \mathrm{C}$ NMR spectrum of compound $3\left(\mathrm{CDCl}_{3}, 100 \mathrm{MHz}\right)$
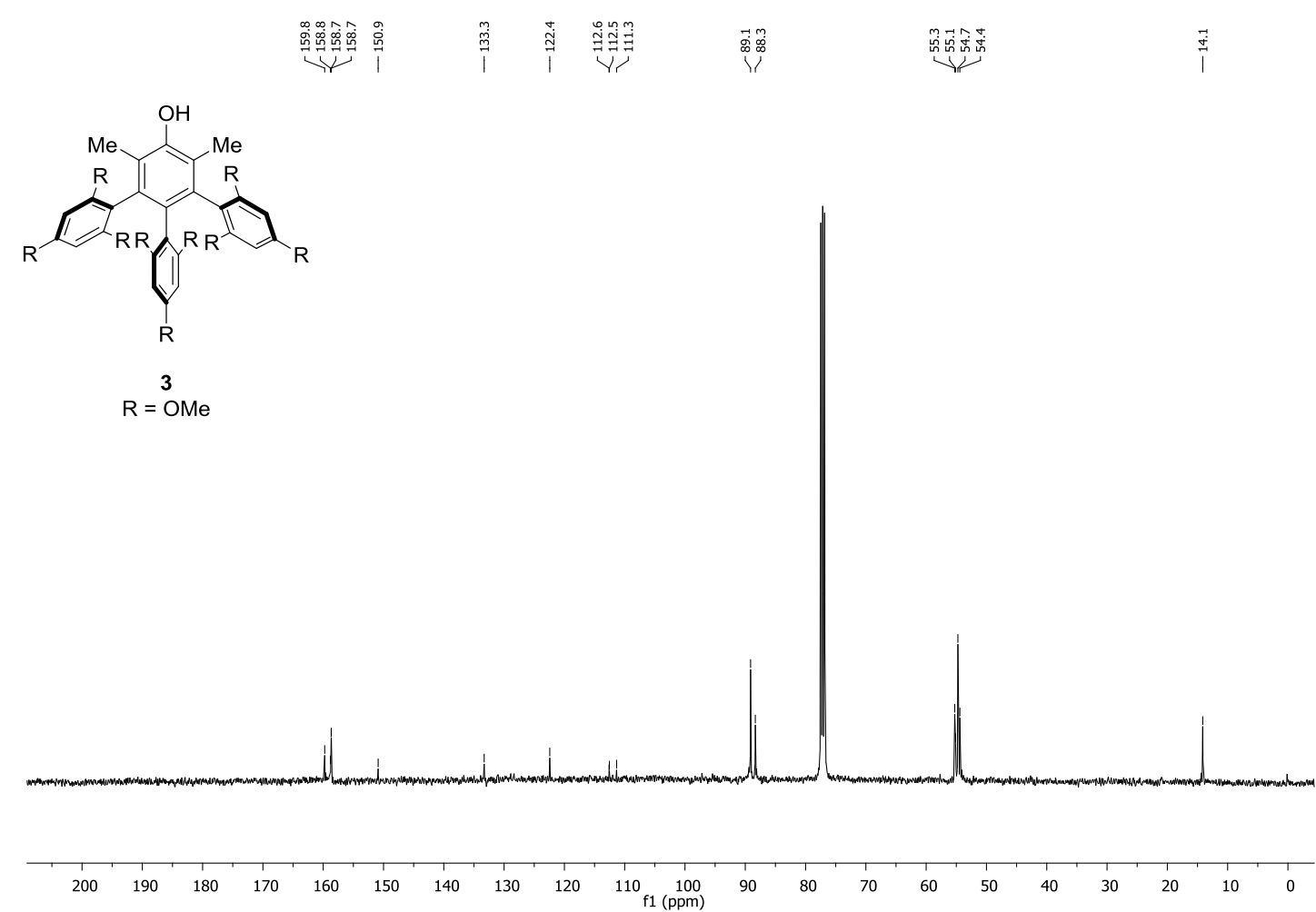
${ }^{1} \mathrm{H}$ NMR spectrum of compound $4\left(\mathrm{CDCl}_{3}, 500 \mathrm{MHz}\right)$
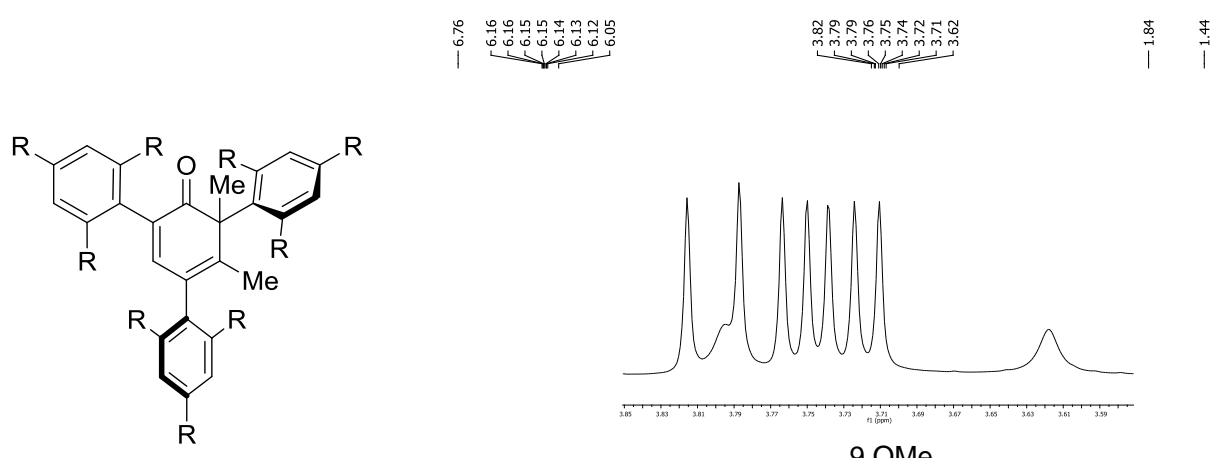

4

$\mathrm{R}=\mathrm{OMe}$
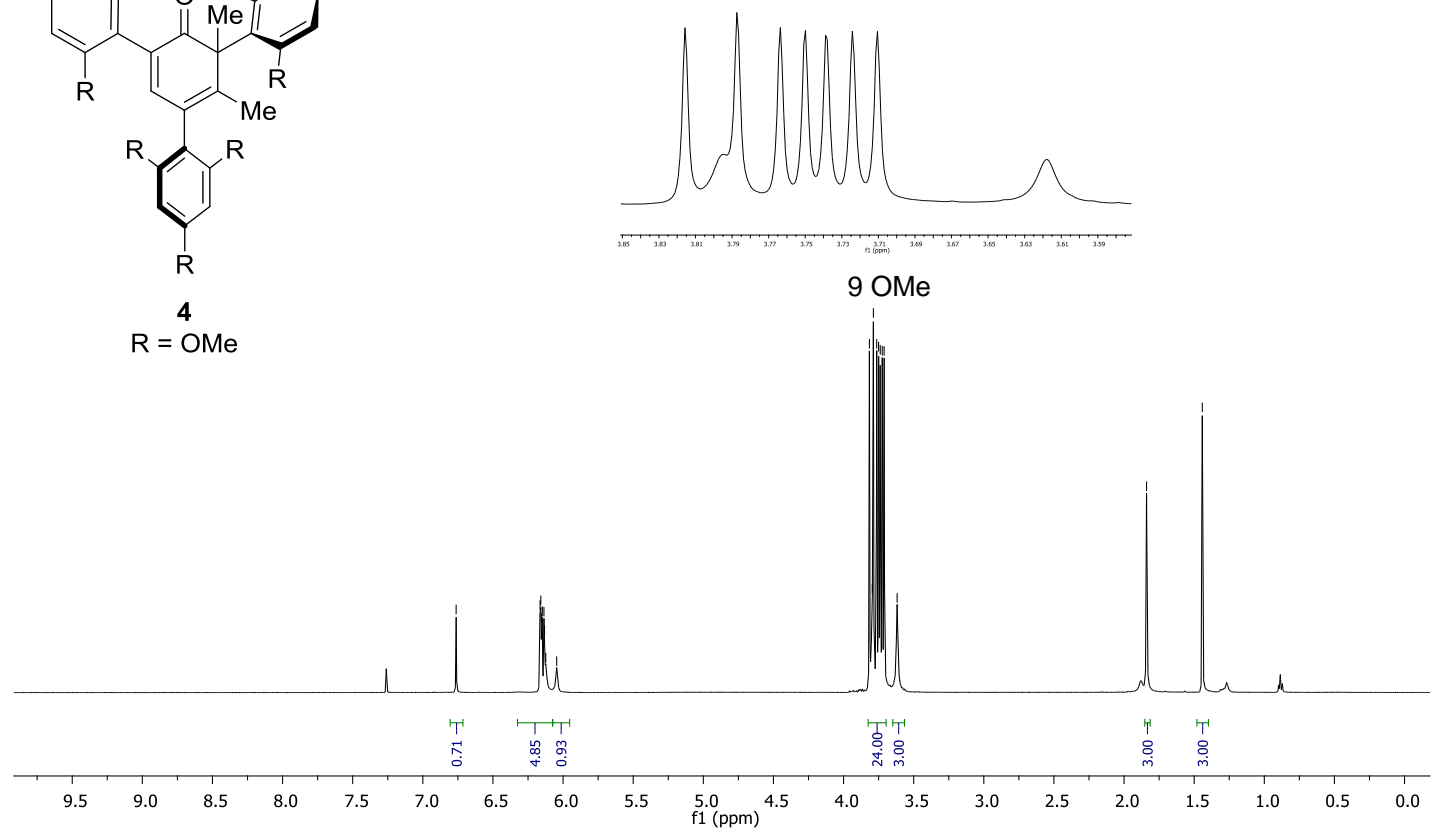

${ }^{13} \mathrm{C}$ NMR spectrum of compound $4\left(\mathrm{CDCl}_{3}, 125 \mathrm{MHz}\right)$
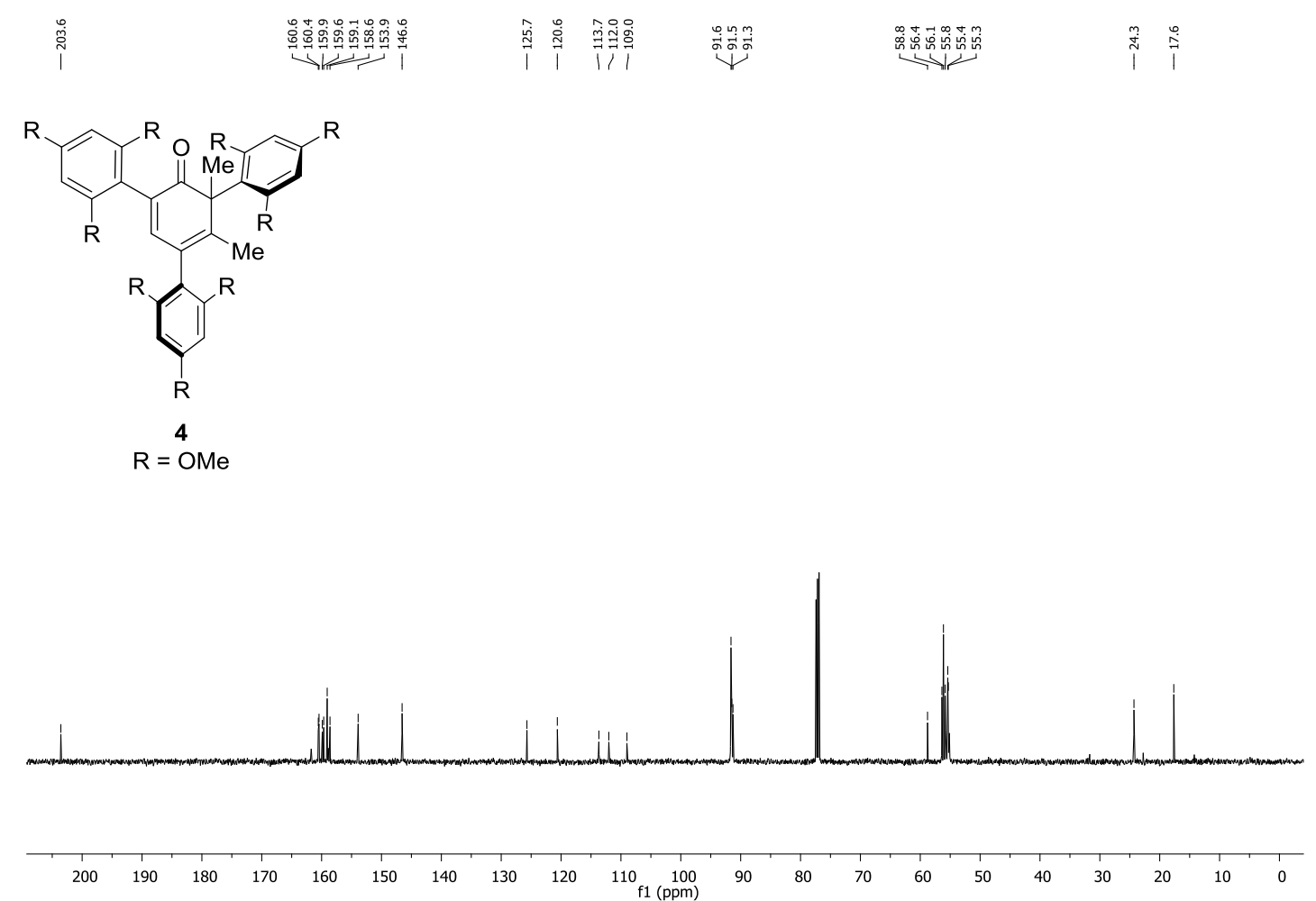
${ }^{1} \mathrm{H}$ NMR spectrum of compound $\mathbf{4 a}\left(\mathrm{CDCl}_{3}, 400 \mathrm{MHz}\right)$

$$
\text { ソัm }
$$
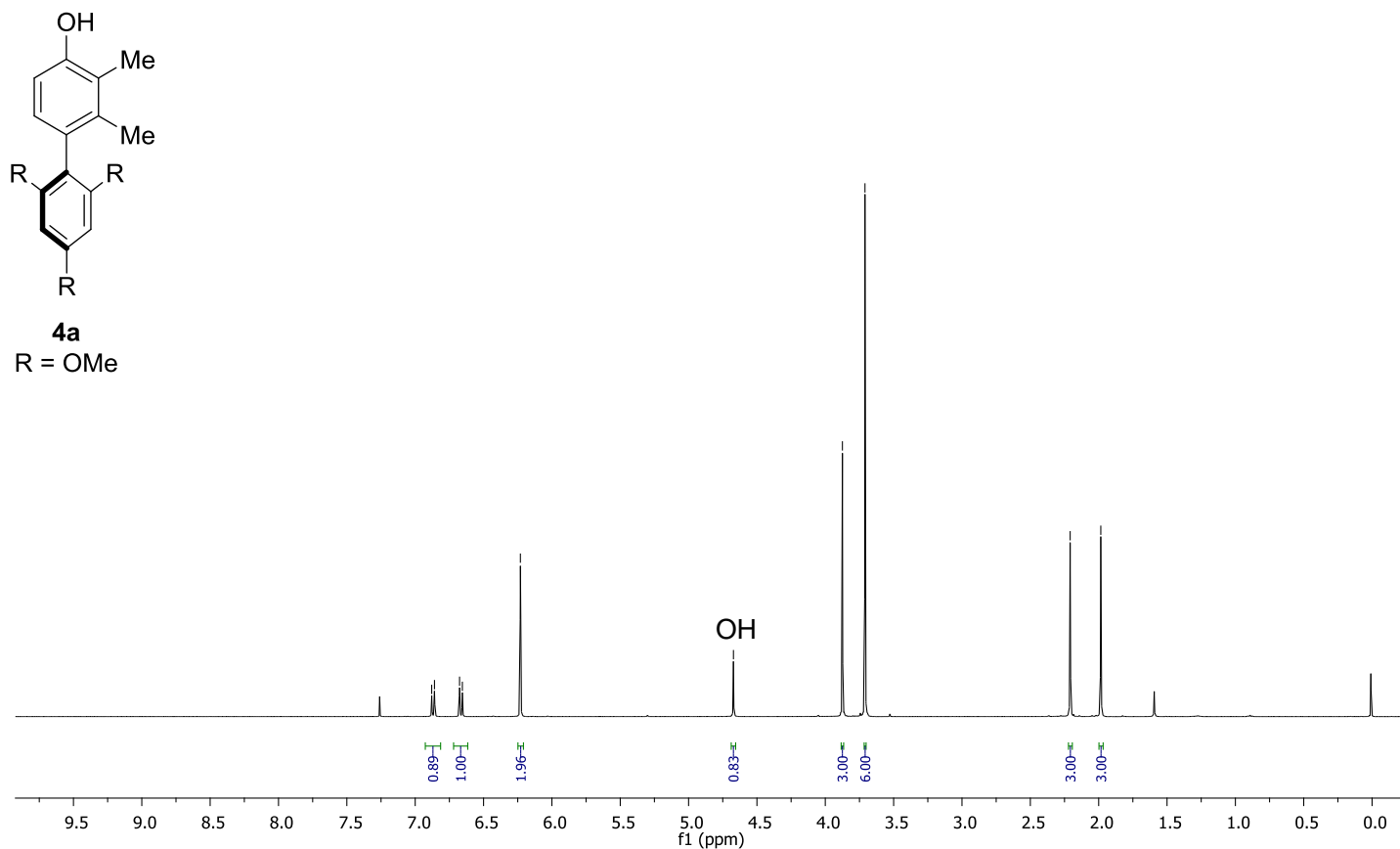

${ }^{13} \mathrm{C}$ NMR spectrum of compound $4 \mathbf{a}\left(\mathrm{CDCl}_{3}, 100 \mathrm{MHz}\right)$

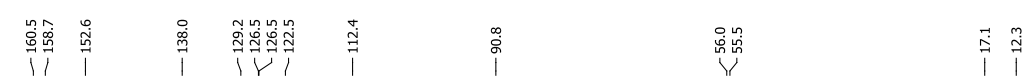
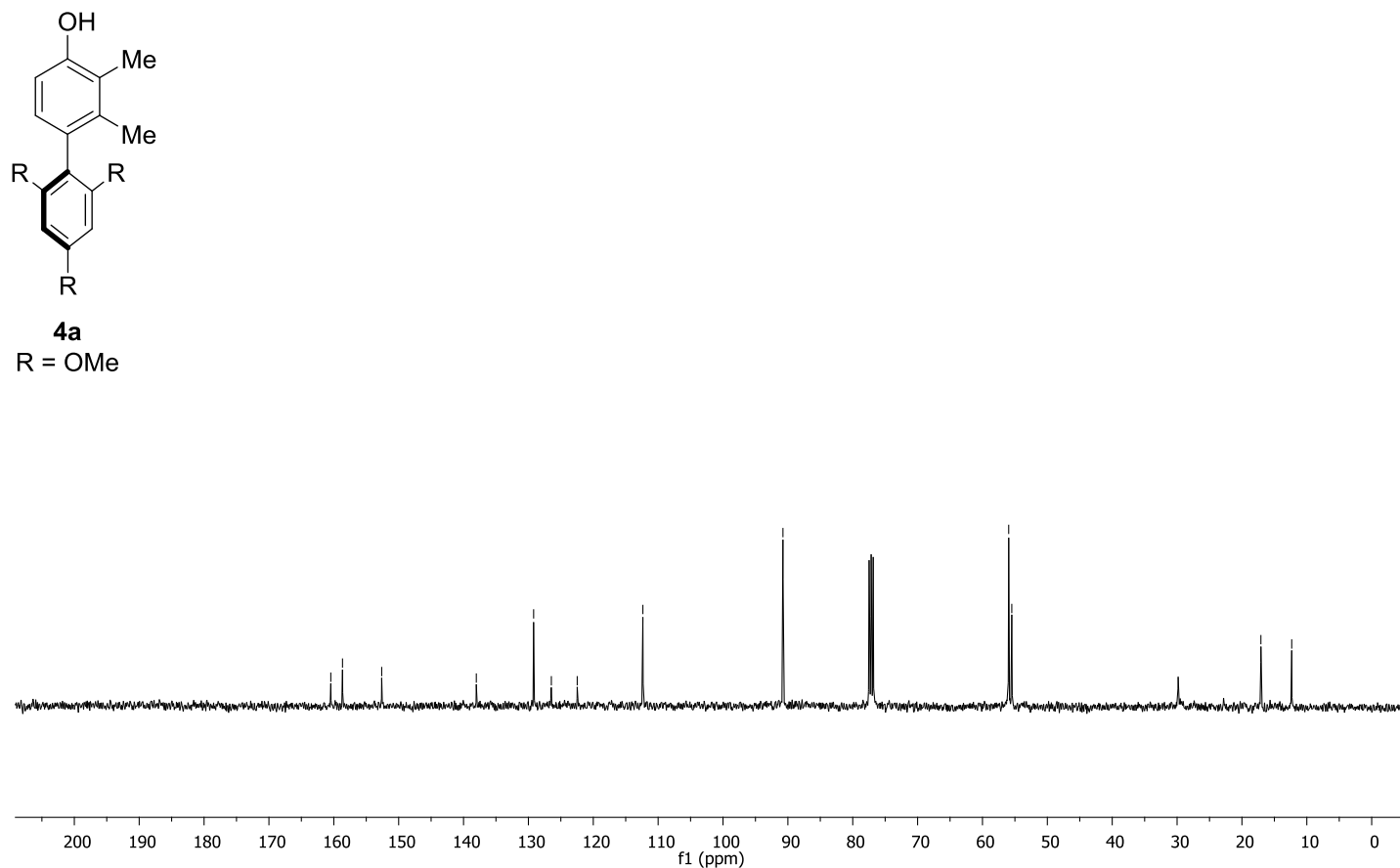
${ }^{1} \mathrm{H}$ NMR spectrum of compound $\mathbf{4 b}\left(\mathrm{CDCl}_{3}, 500 \mathrm{MHz}\right)$
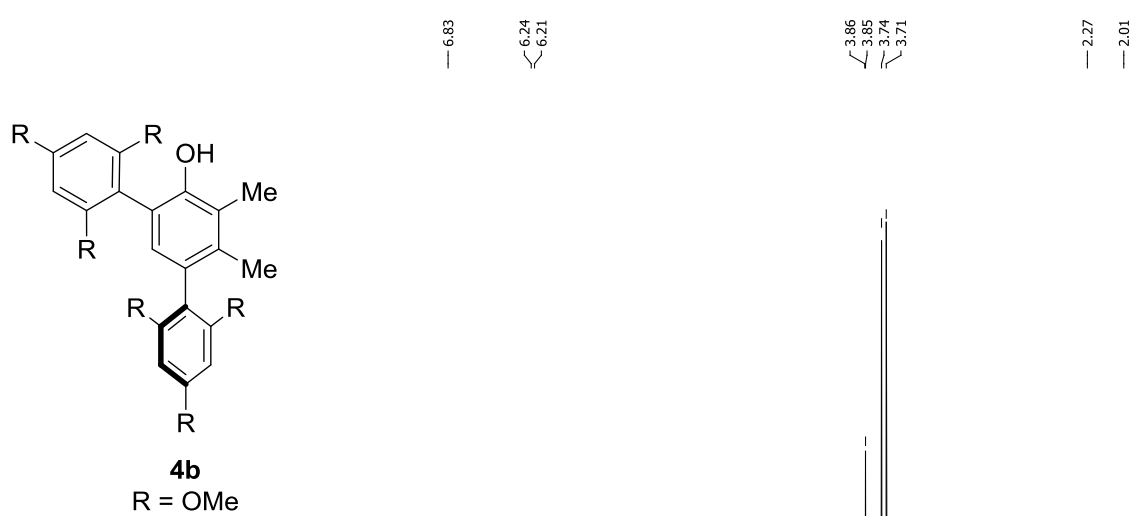

iิ îं

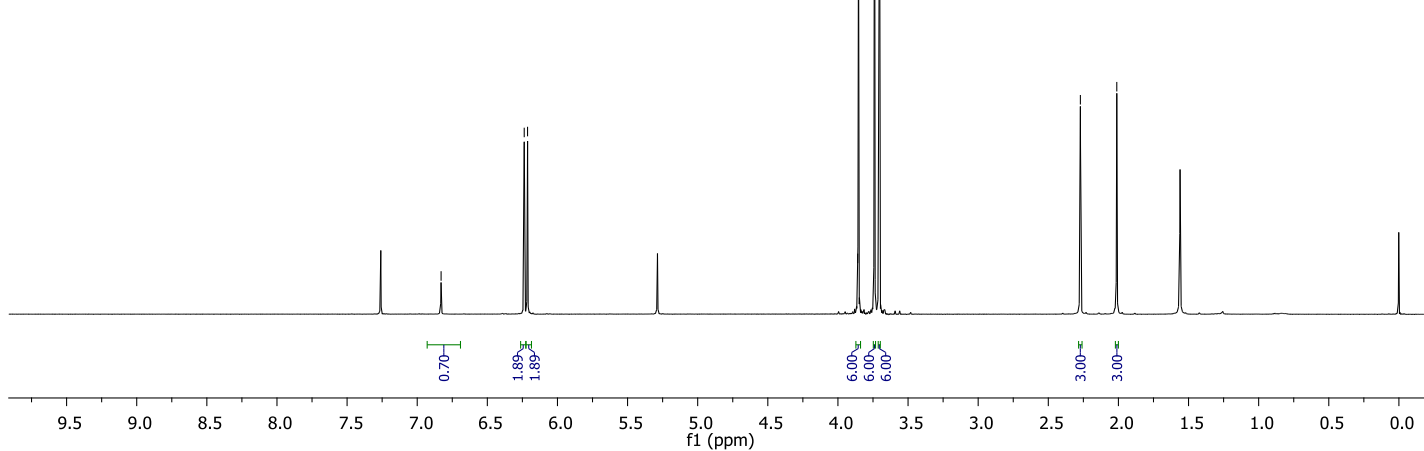

${ }^{13} \mathrm{C}$ NMR spectrum of compound $\mathbf{4 b}\left(\mathrm{CDCl}_{3}, 100 \mathrm{MHz}\right)$
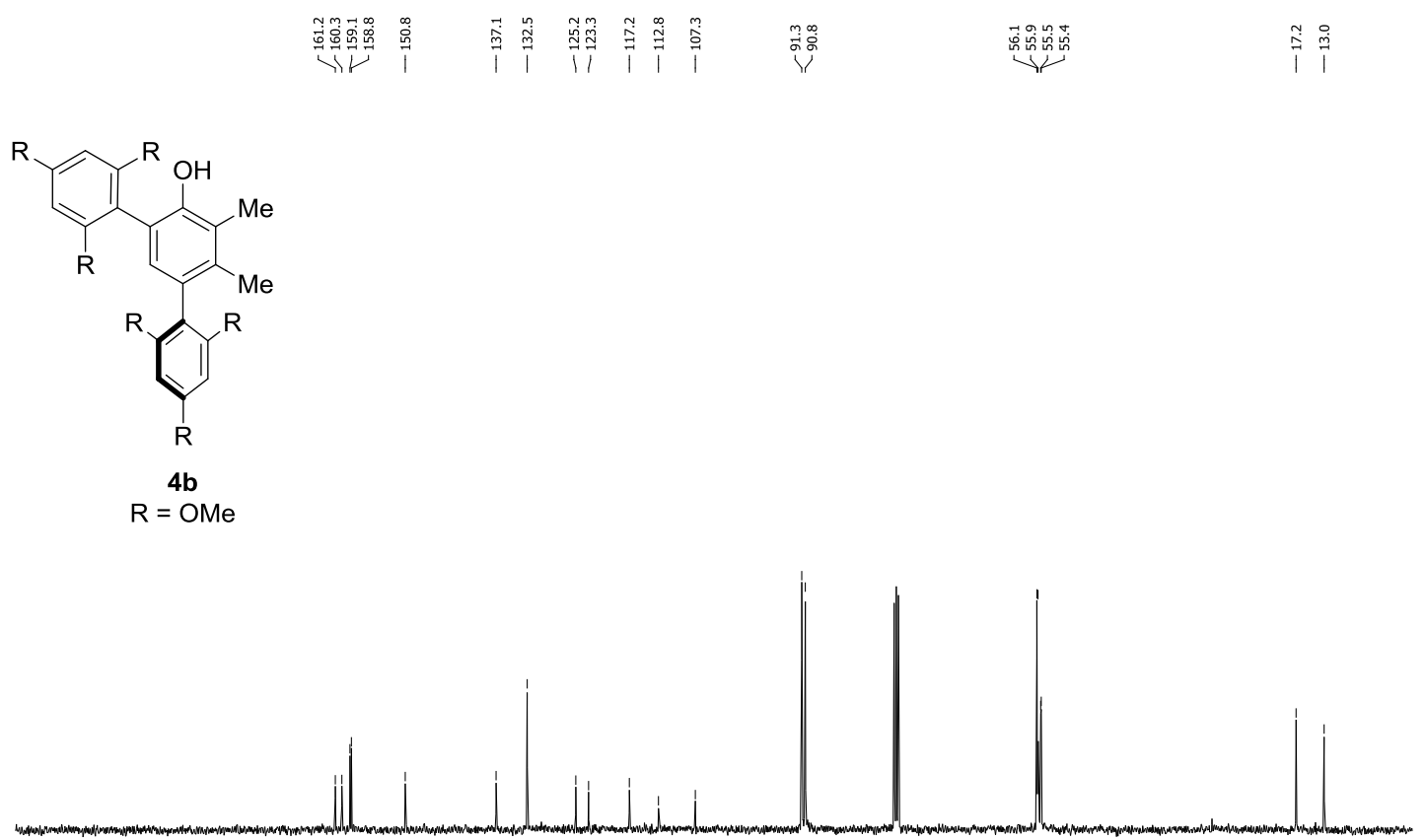

$\begin{array}{llllllllllllllllllllllll}200 & 190 & 180 & 170 & 160 & 150 & 140 & 130 & 120 & 110 & 100 & 90 & 80 & 70 & 60 & 50 & 40 & 30 & 20 & 10 & 0\end{array}$ 
${ }^{1} \mathrm{H}$ NMR spectrum of compound $5\left(\mathrm{CDCl}_{3}, 400 \mathrm{MHz}\right)$

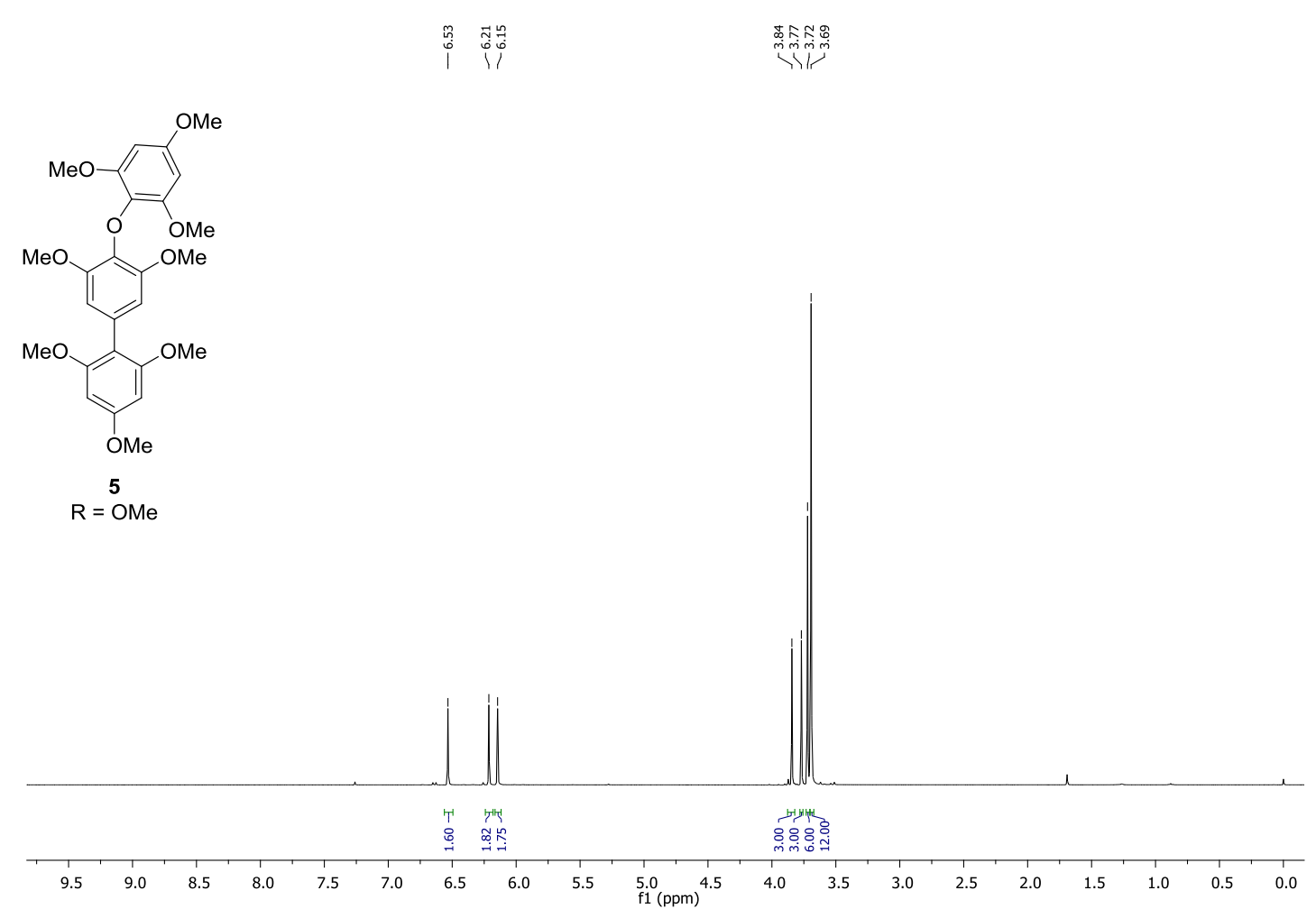

${ }^{13} \mathrm{C}$ NMR spectrum of compound $5\left(\mathrm{CDCl}_{3}, 100 \mathrm{MHz}\right)$
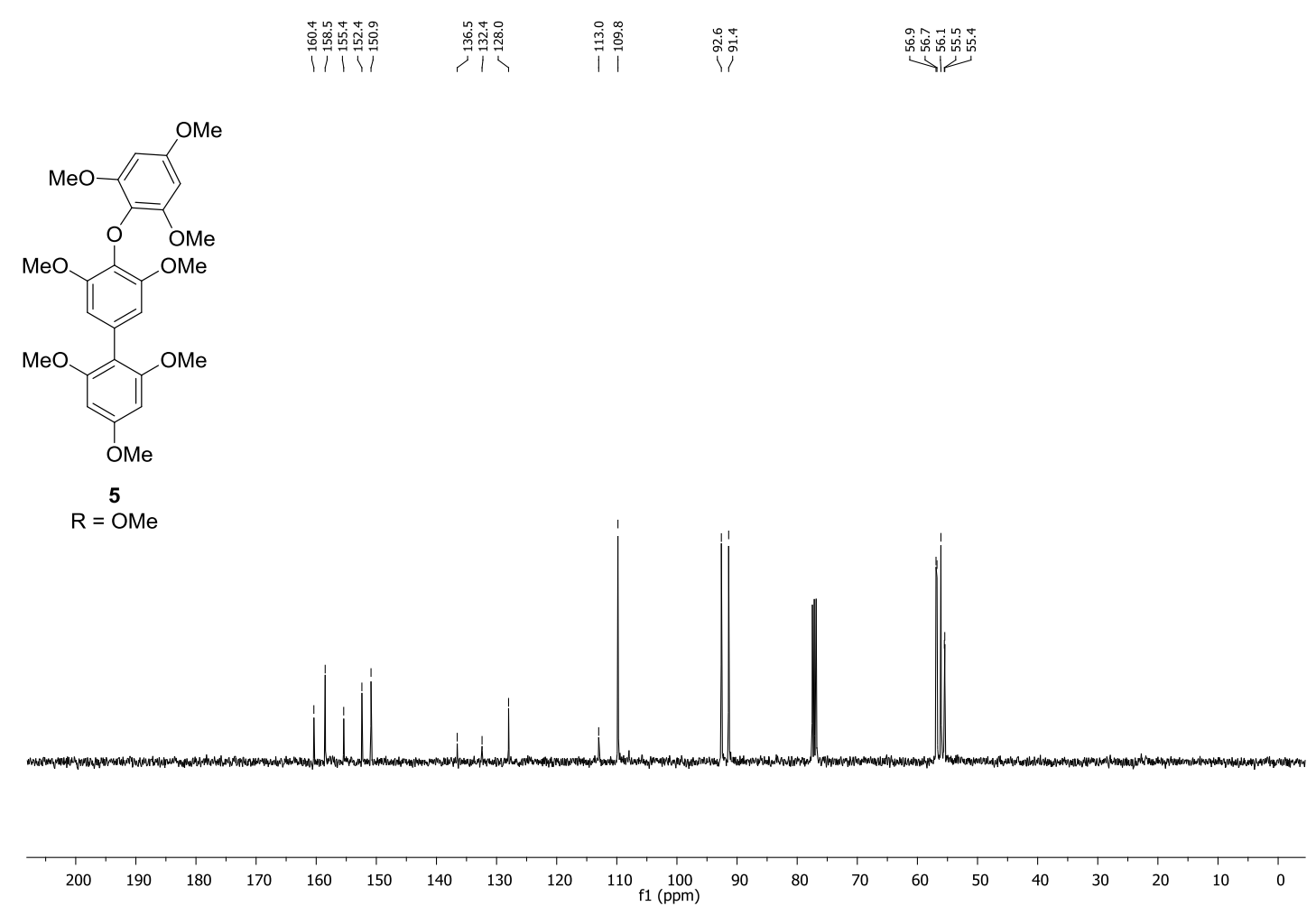
${ }^{1} \mathrm{H}$ NMR spectrum of compound $6\left(\mathrm{CDCl}_{3}, 400 \mathrm{MHz}\right)$

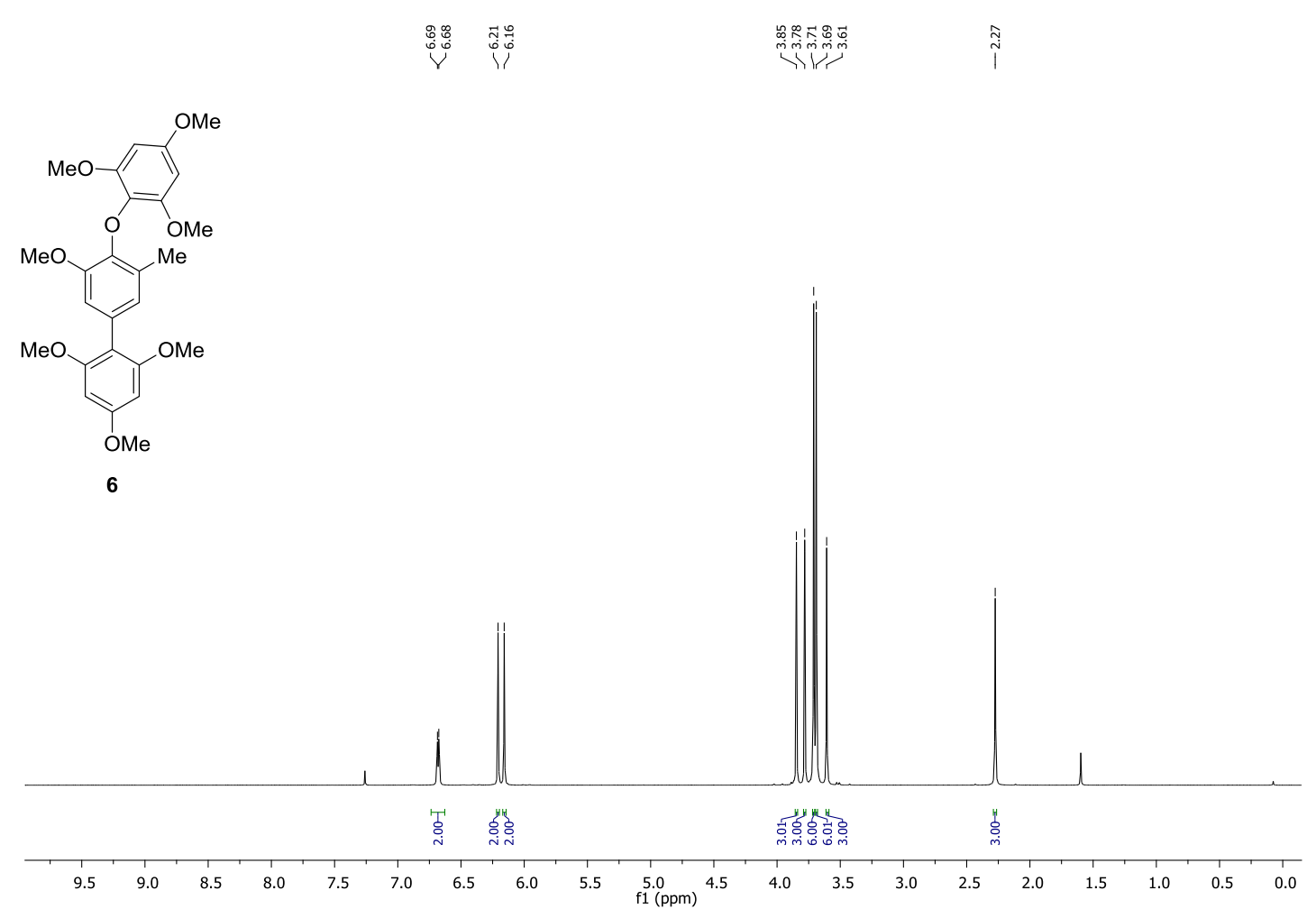

${ }^{13} \mathrm{C}$ NMR spectrum of compound $6\left(\mathrm{CDCl}_{3}, 100 \mathrm{MHz}\right)$

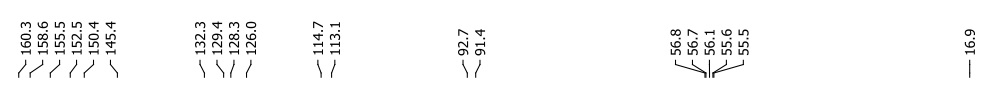
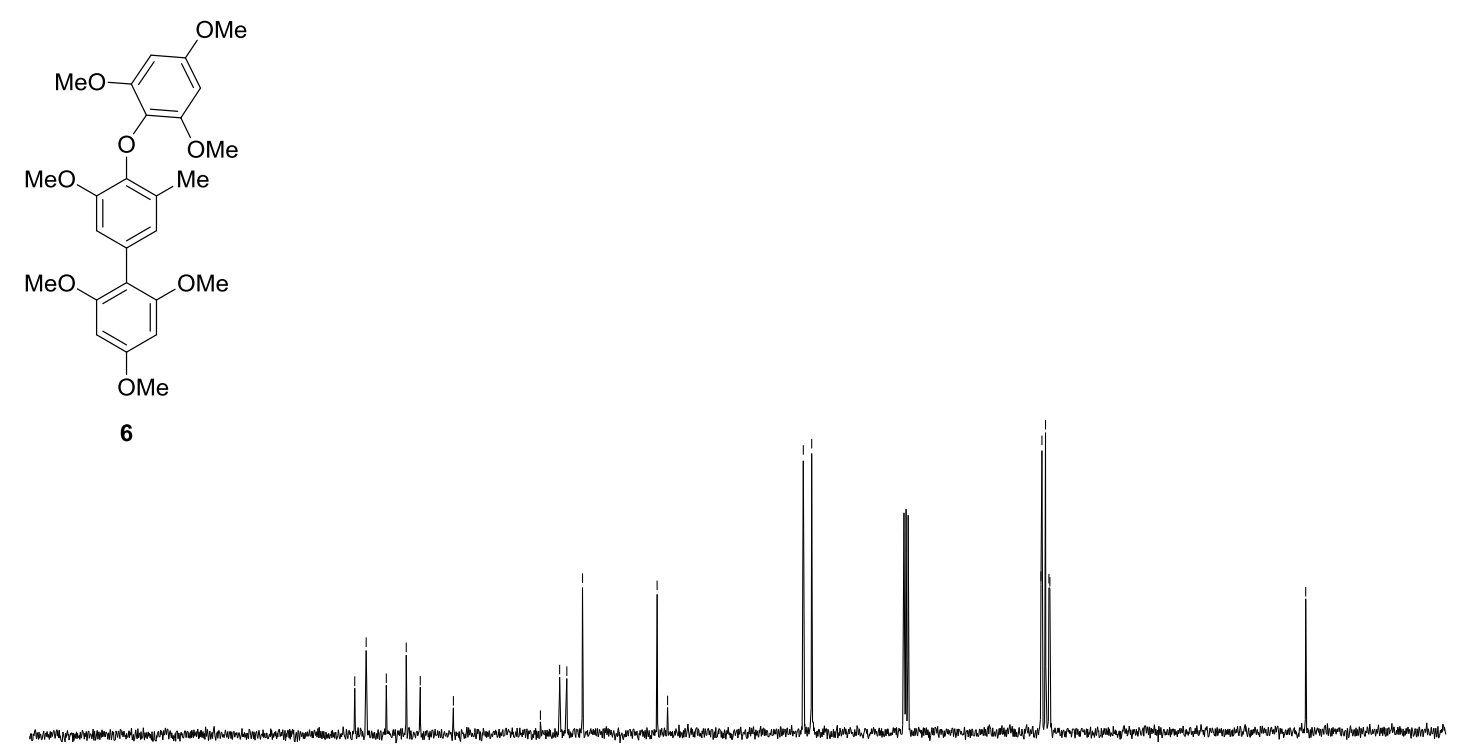

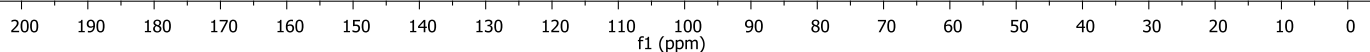


${ }^{1} \mathrm{H}$ NMR spectrum of compound $7\left(\mathrm{CDCl}_{3}, 400 \mathrm{MHz}\right)$

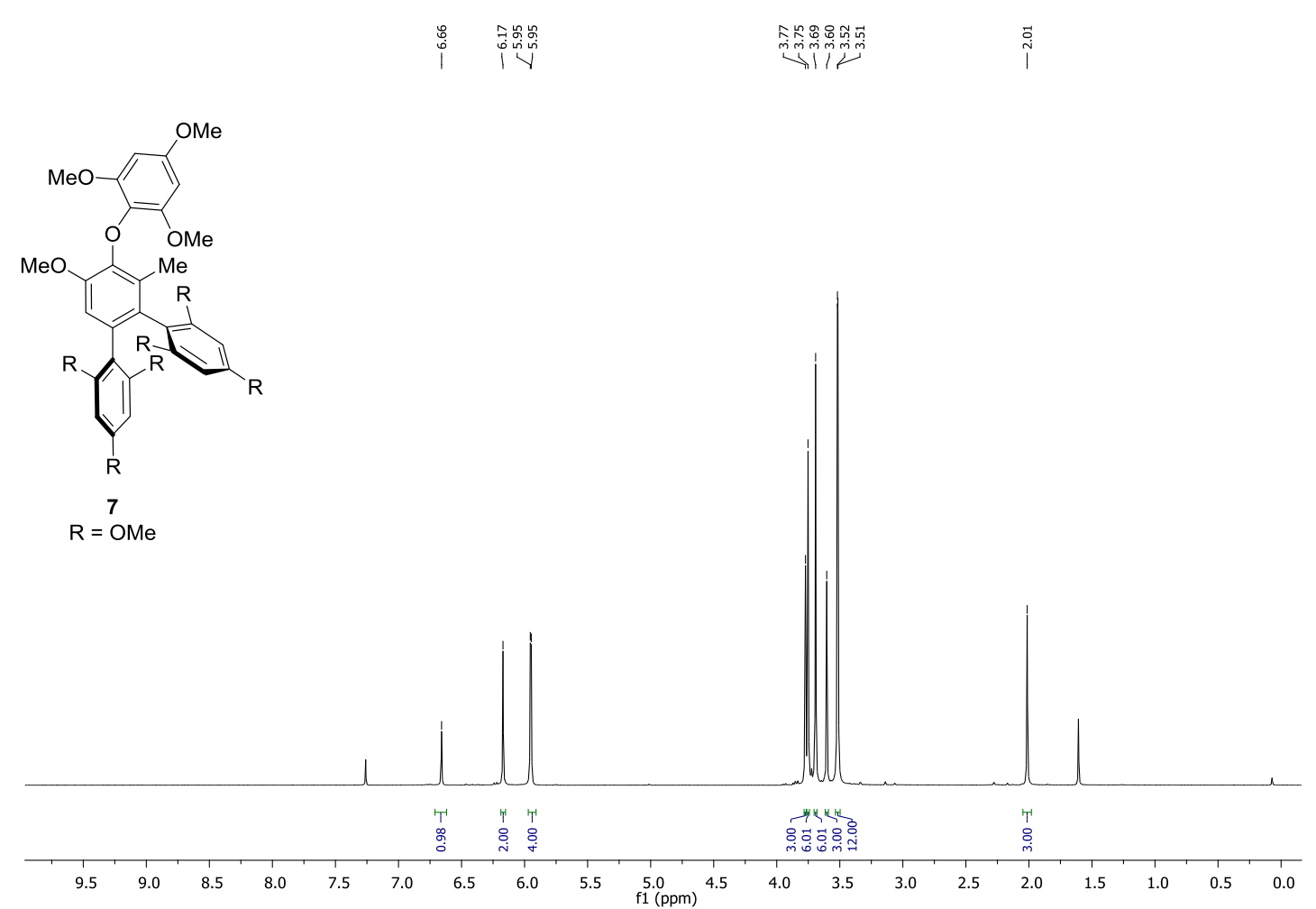

${ }^{13} \mathrm{C}$ NMR spectrum of compound $7\left(\mathrm{CDCl}_{3}, 100 \mathrm{MHz}\right)$
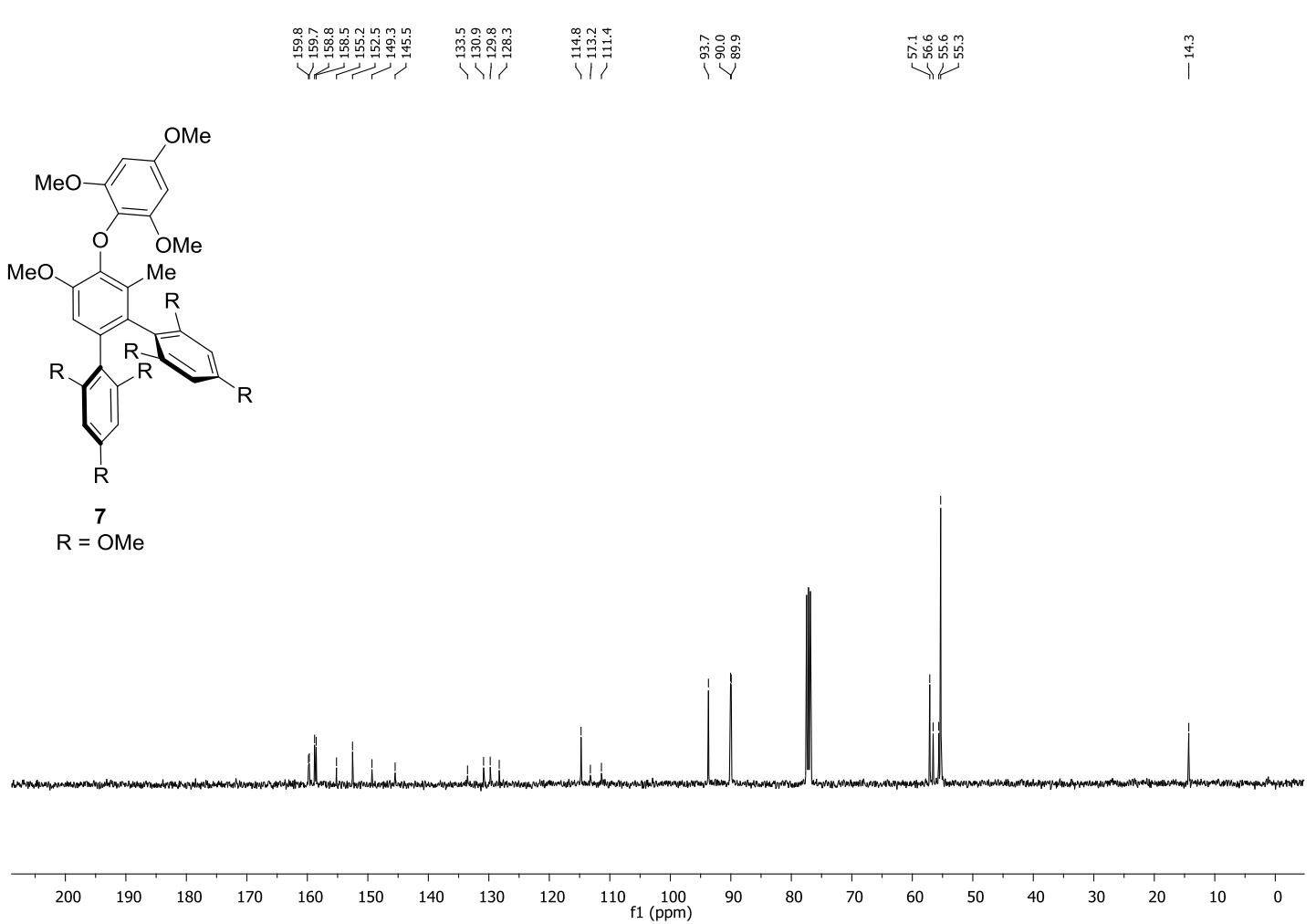
${ }^{1} \mathrm{H}$ NMR spectrum of compound $\mathbf{8}\left(\mathrm{CDCl}_{3}, 500 \mathrm{MHz}\right)$

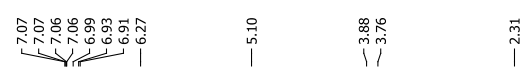
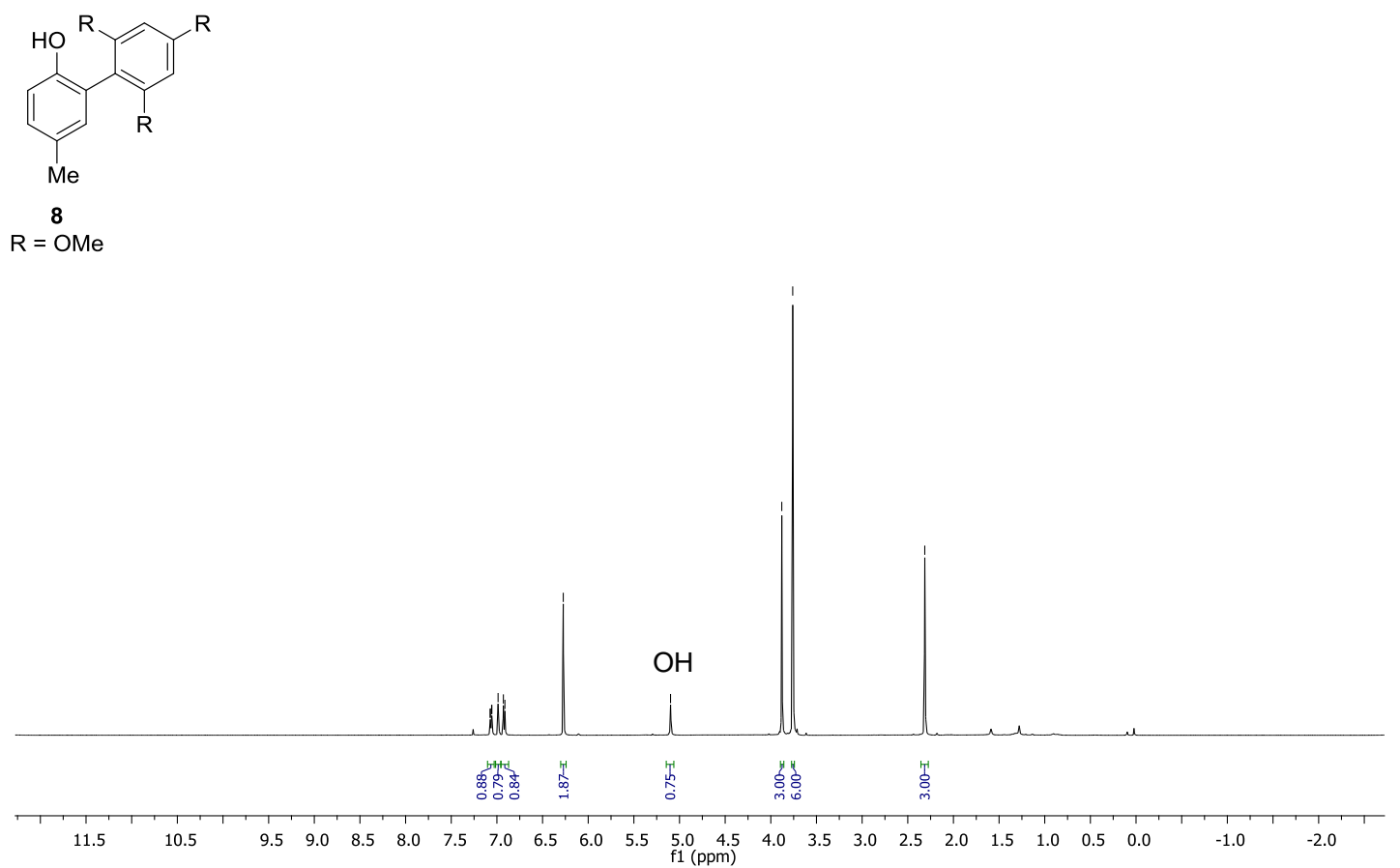

${ }^{13} \mathrm{C}$ NMR spectrum of compound $\mathbf{8}\left(\mathrm{CDCl}_{3}, 125 \mathrm{MHz}\right)$
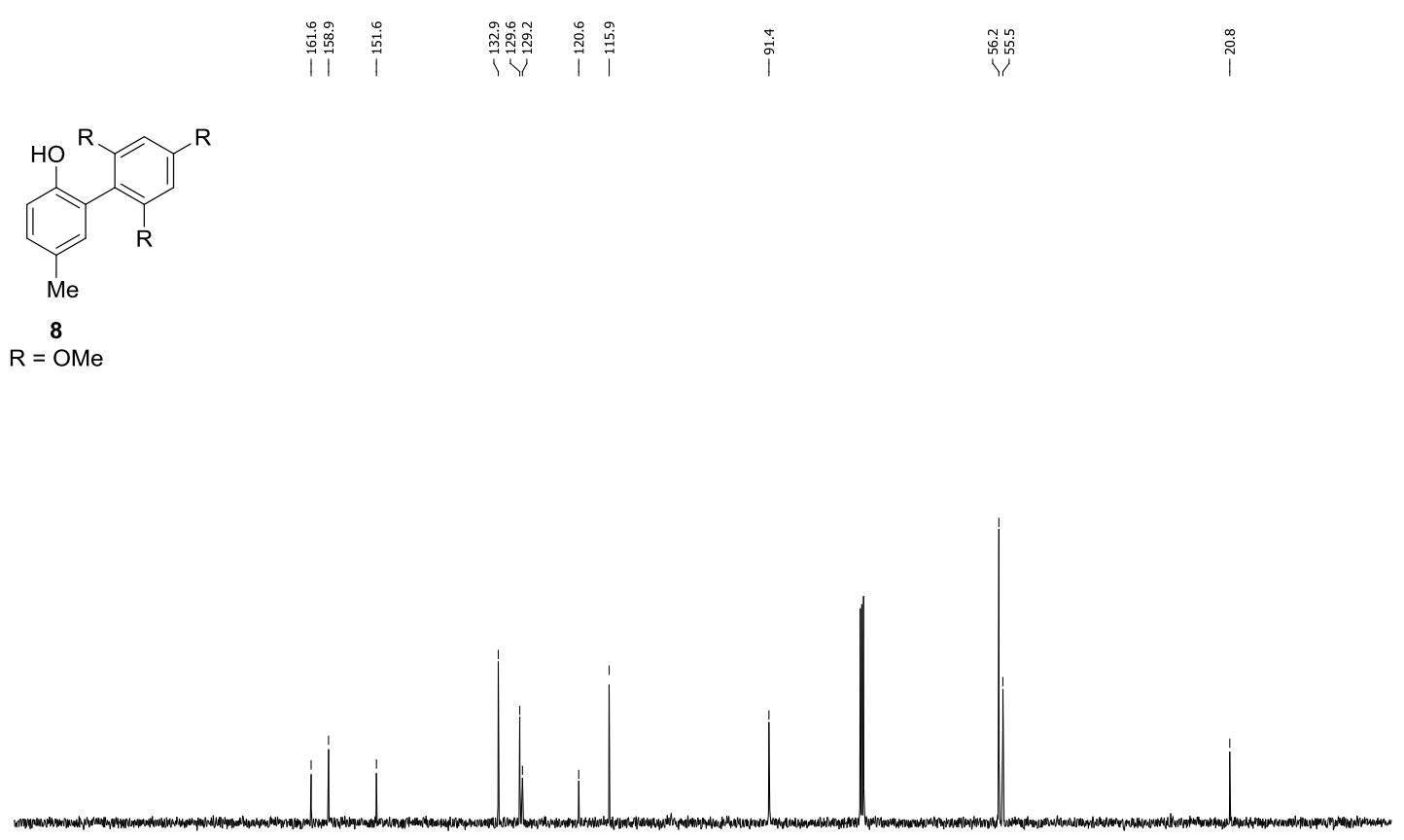

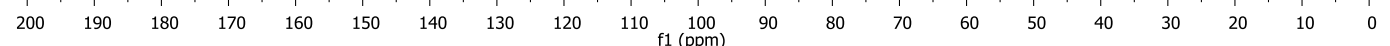


${ }^{1} \mathrm{H}$ NMR spectrum of compound $9\left(\mathrm{CDCl}_{3}, 400 \mathrm{MHz}\right)$

盟照影

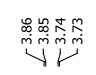

i
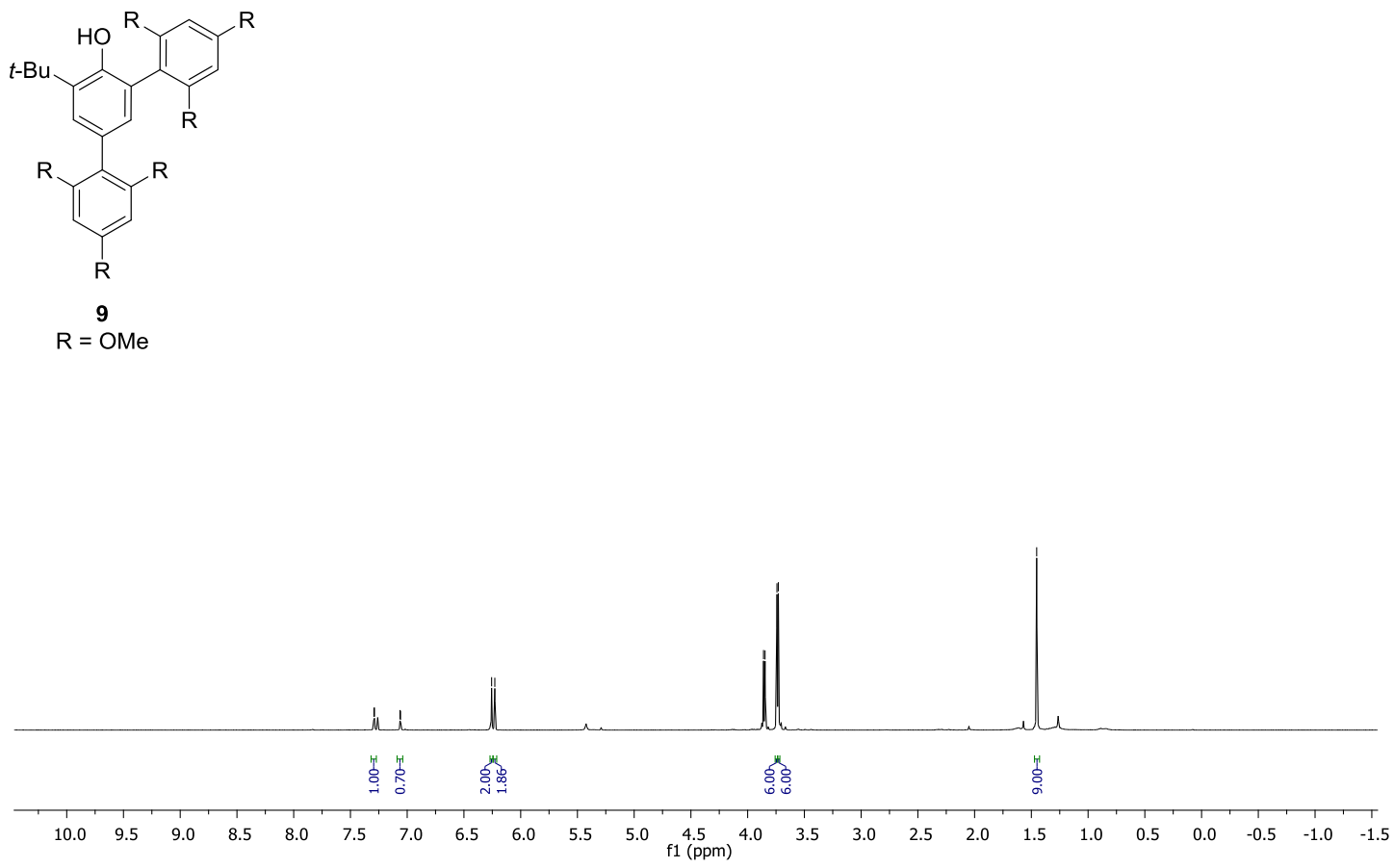

${ }^{13} \mathrm{C} \mathrm{NMR}$ spectrum of compound $9\left(\mathrm{CDCl}_{3}, 100 \mathrm{MHz}\right)$

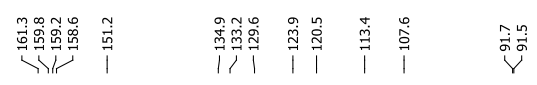

Ur

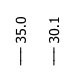
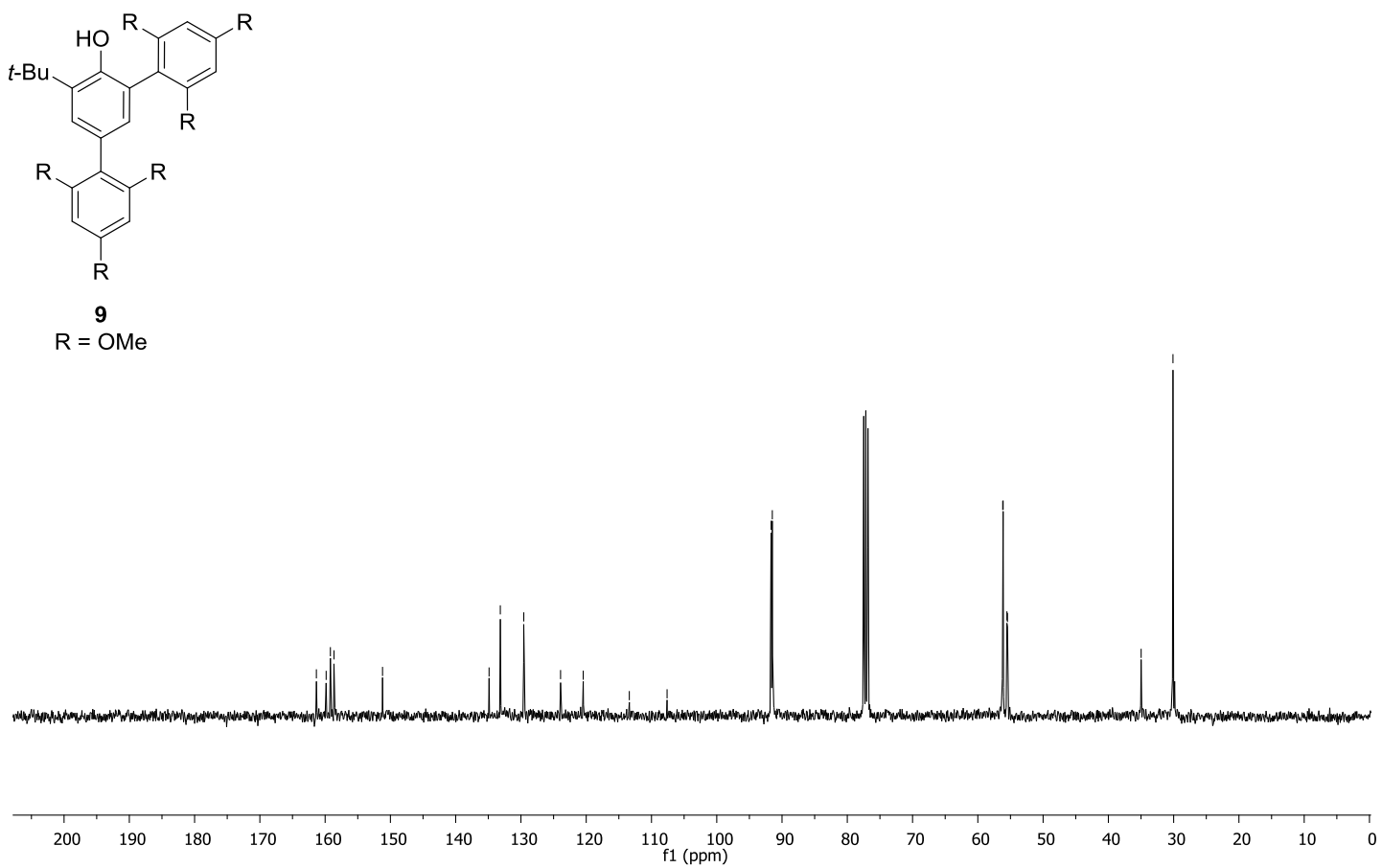

S38 
${ }^{1} \mathrm{H}$ NMR spectrum of compound $10\left(\mathrm{CDCl}_{3}, 500 \mathrm{MHz}\right)$

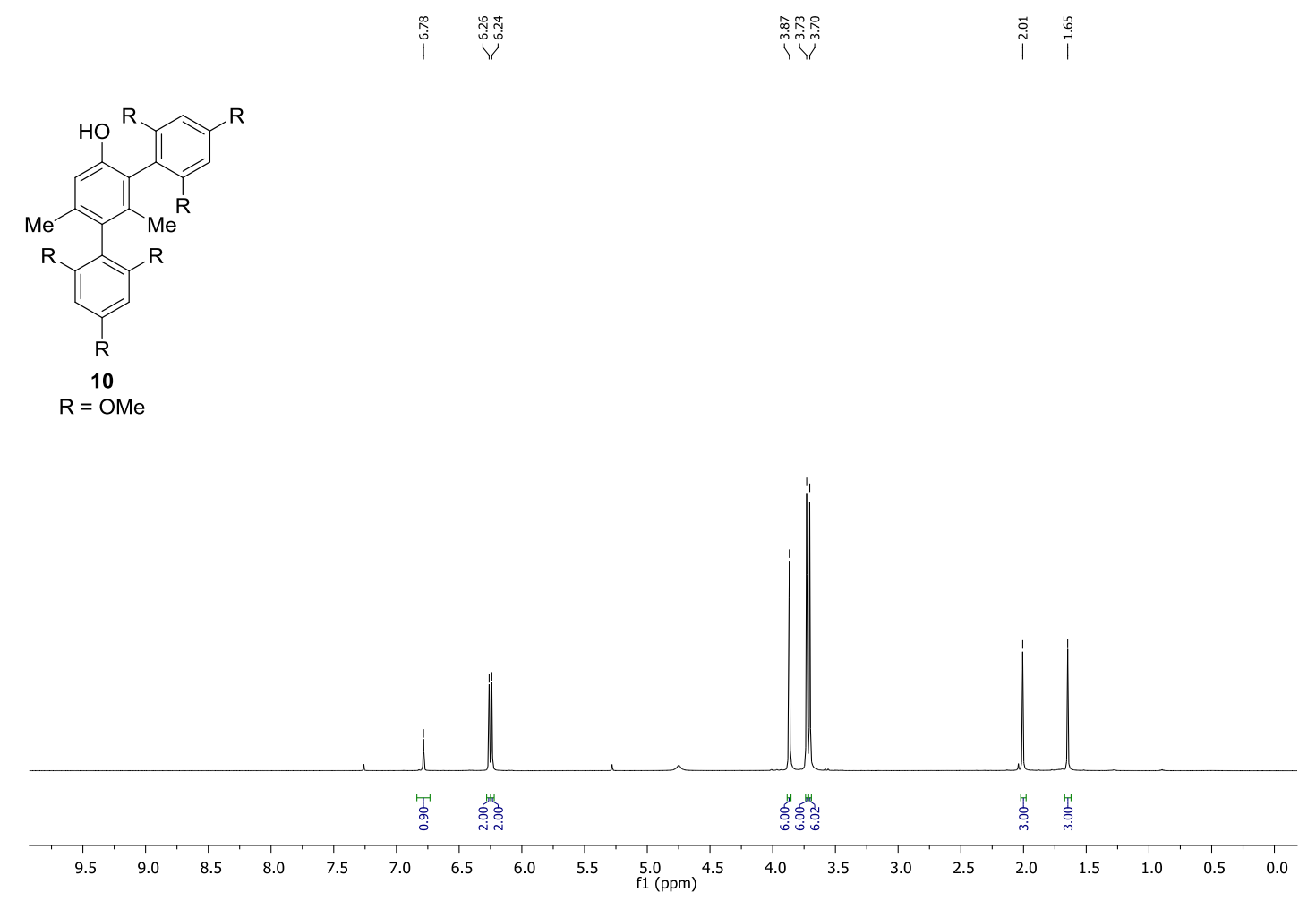

${ }^{13} \mathrm{C}$ NMR spectrum of compound $\mathbf{1 0}\left(\mathrm{CDCl}_{3}, 125 \mathrm{MHz}\right)$

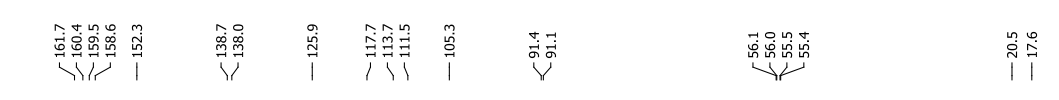
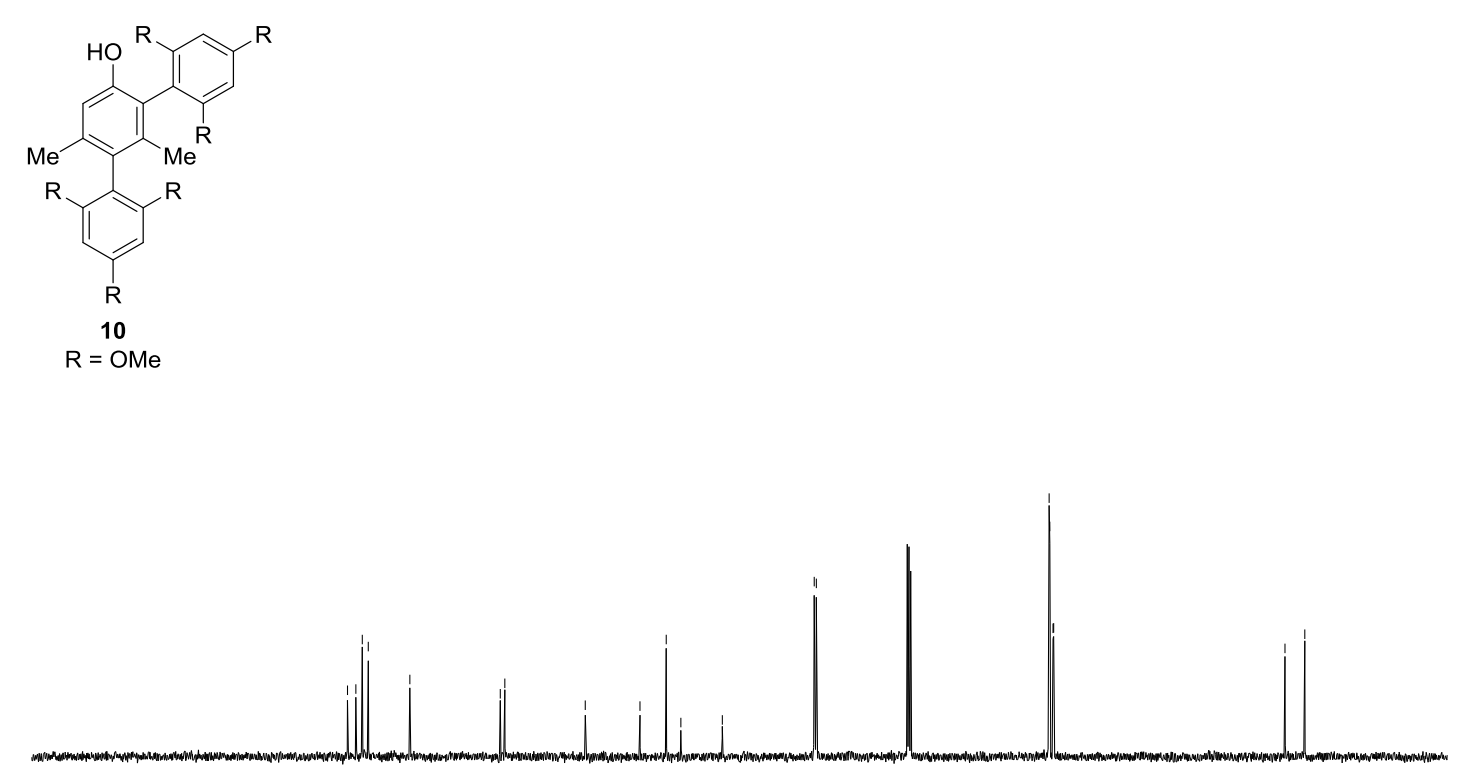

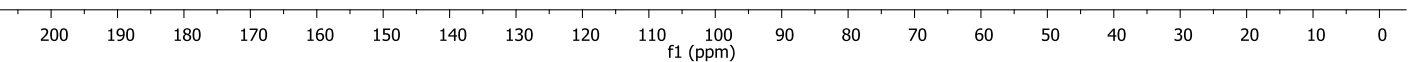


${ }^{1} \mathrm{H}$ NMR spectrum of compound $11\left(\mathrm{CDCl}_{3}, 400 \mathrm{MHz}\right)$

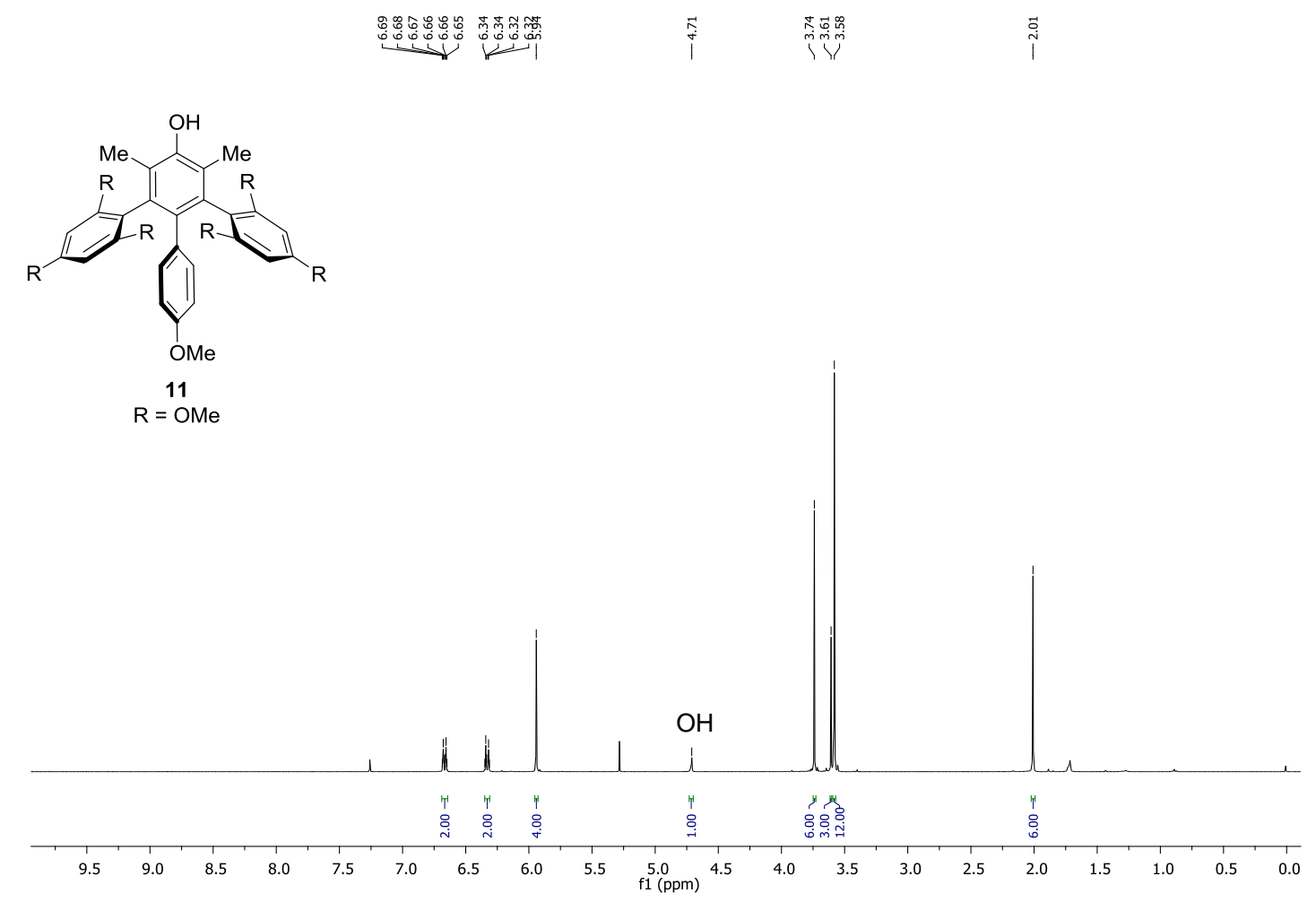

${ }^{13} \mathrm{C}$ NMR spectrum of compound $\mathbf{1 1}\left(\mathrm{CDCl}_{3}, 100 \mathrm{MHz}\right)$
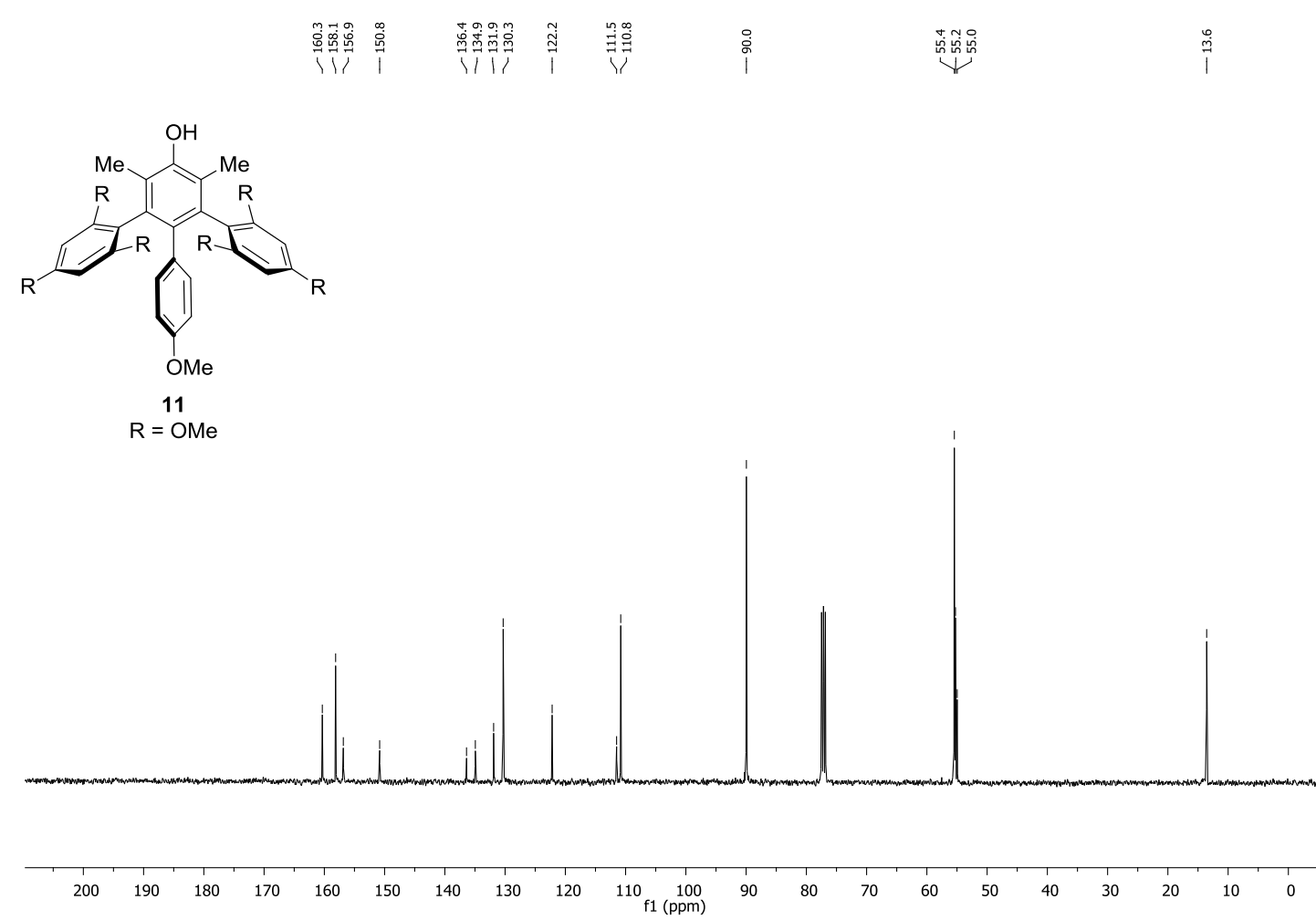
${ }^{1} \mathrm{H}$ NMR spectrum of compound $12\left(\mathrm{CDCl}_{3}, 400 \mathrm{MHz}\right)$

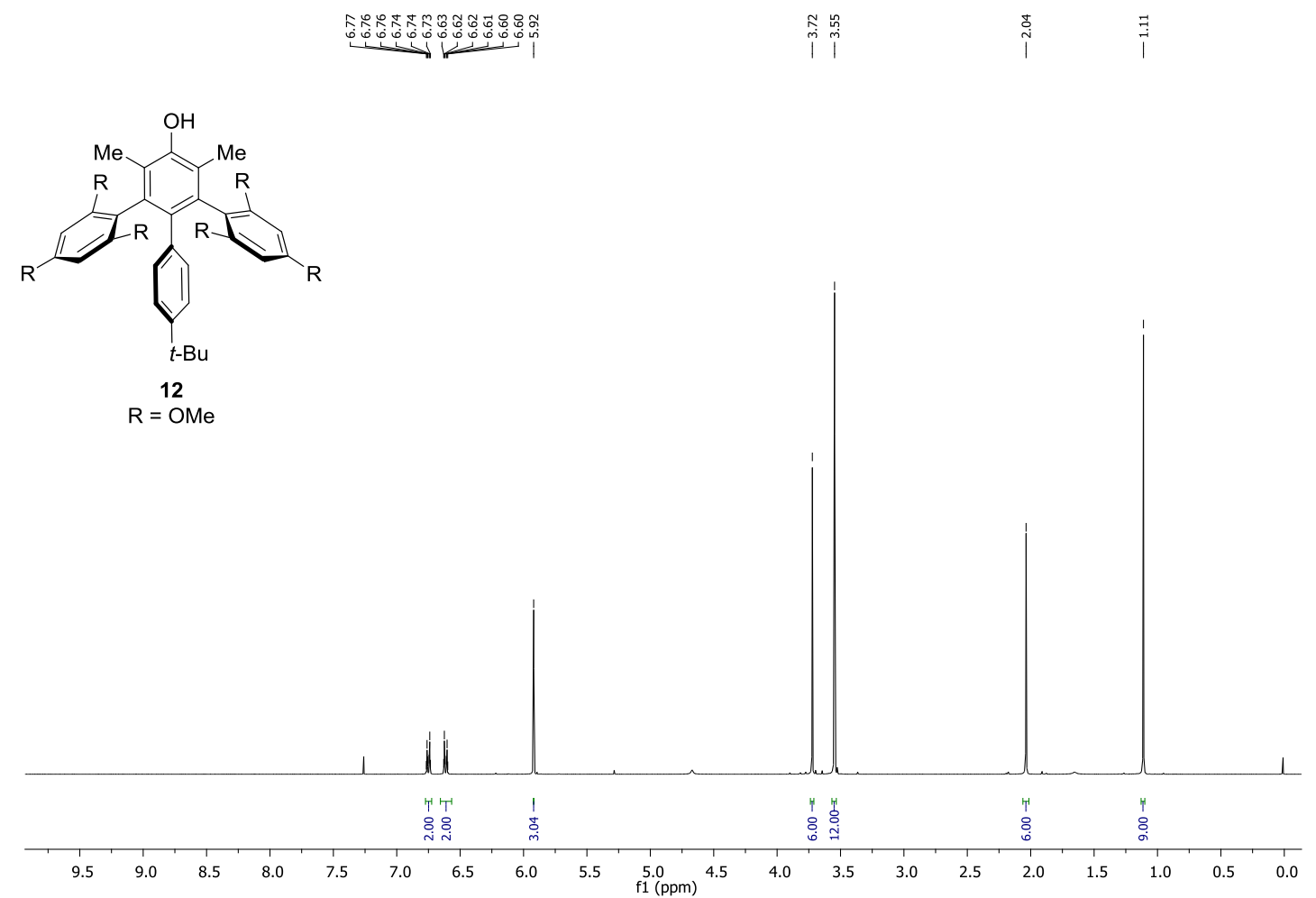

${ }^{13} \mathrm{C}$ NMR spectrum of compound $12\left(\mathrm{CDCl}_{3}, 100 \mathrm{MHz}\right)$

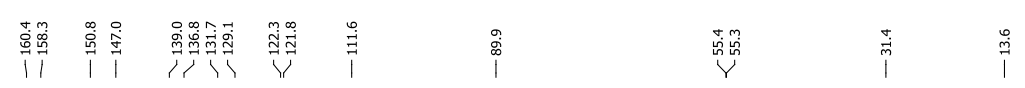
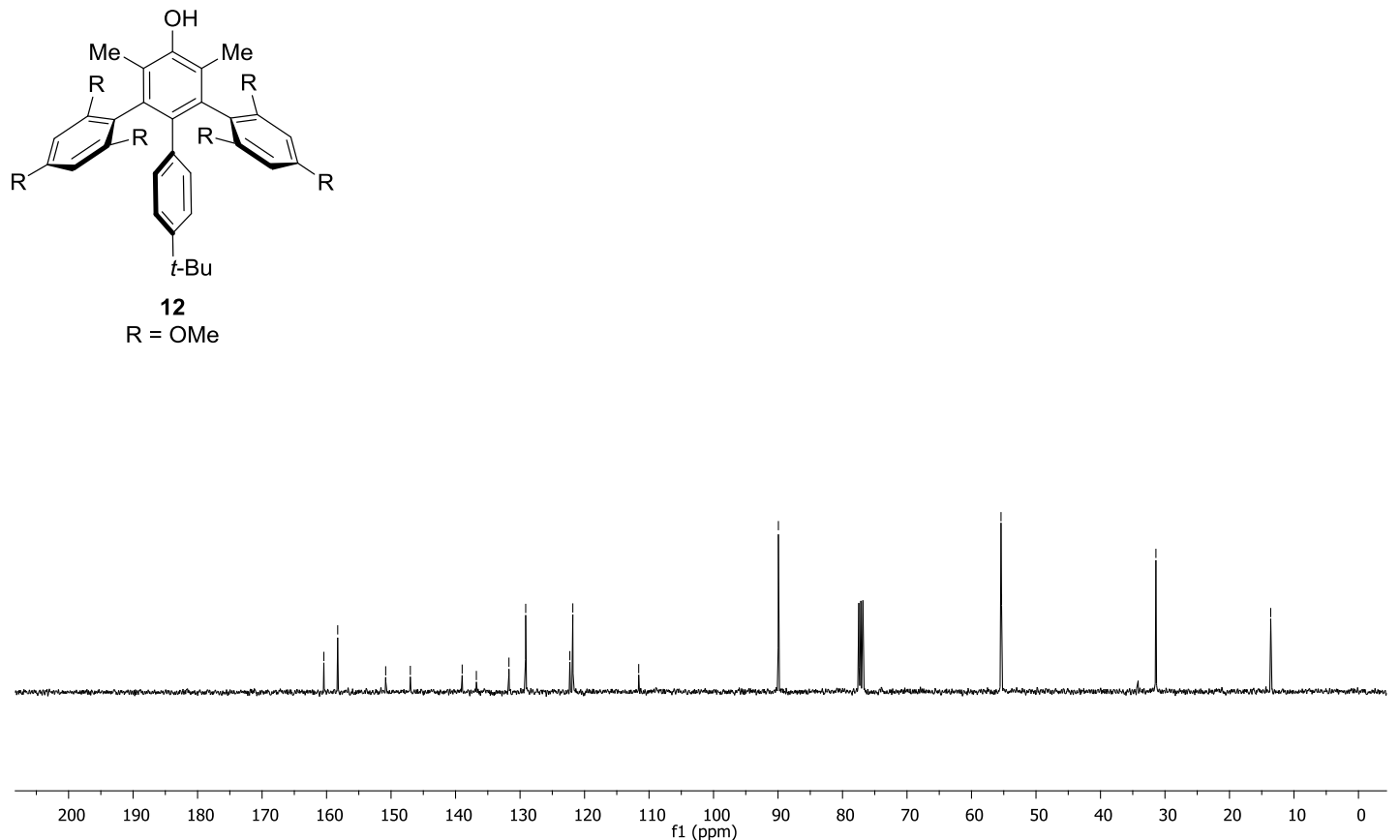
${ }^{1} \mathrm{H}$ NMR spectrum of compound $13\left(\mathrm{CDCl}_{3}, 400 \mathrm{MHz}\right)$
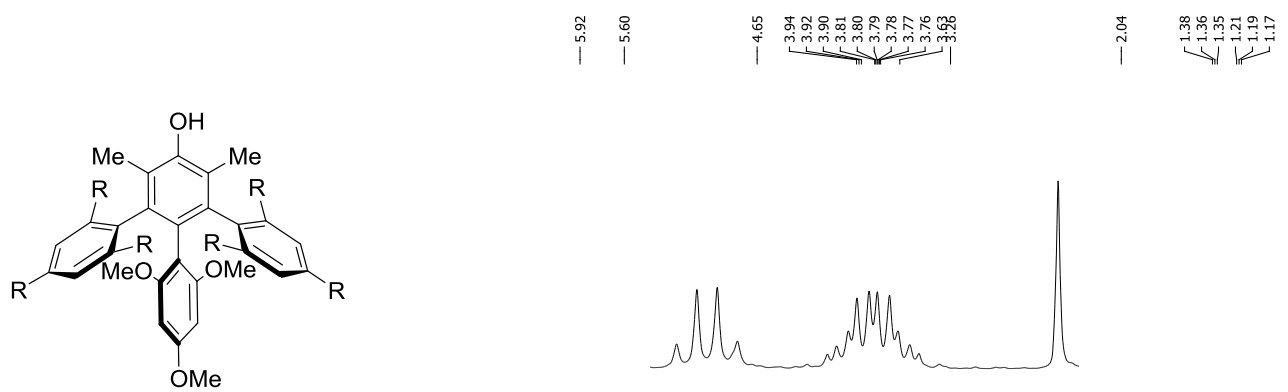

13
$\mathrm{R}=\mathrm{OEt}$
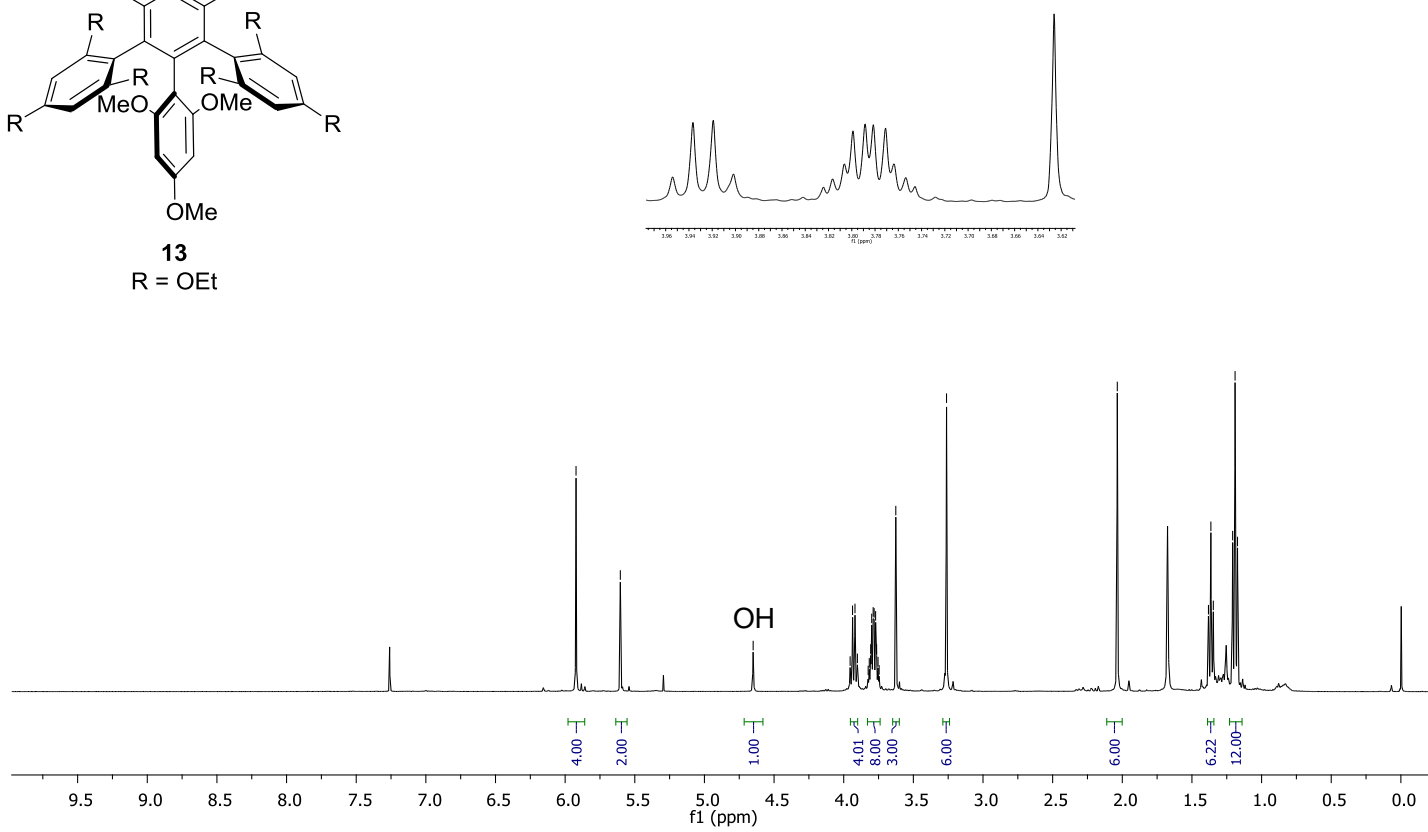

${ }^{13} \mathrm{C}$ NMR spectrum of compound $13\left(\mathrm{CDCl}_{3}, 100 \mathrm{MHz}\right)$
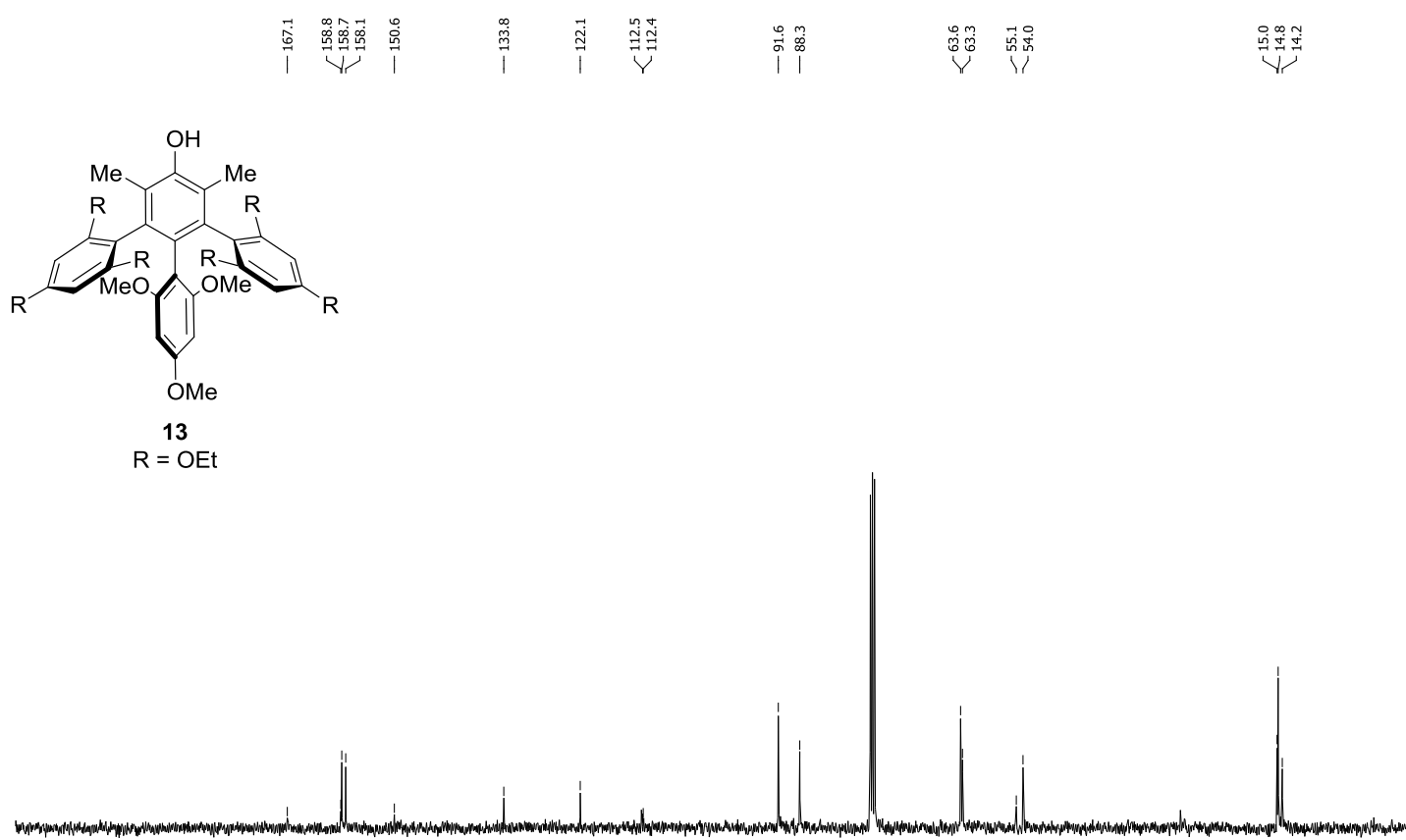

$\begin{array}{lllllllllllllllllllll}200 & 190 & 180 & 170 & 160 & 150 & 140 & 130 & 120 & 110 & 100 & 90 & 80 & 70 & 60 & 50 & 40 & 30 & 20 & 10 & 0\end{array}$ 
${ }^{1} \mathrm{H}$ NMR spectrum of compound $14\left(\mathrm{CDCl}_{3}, 500 \mathrm{MHz}\right)$
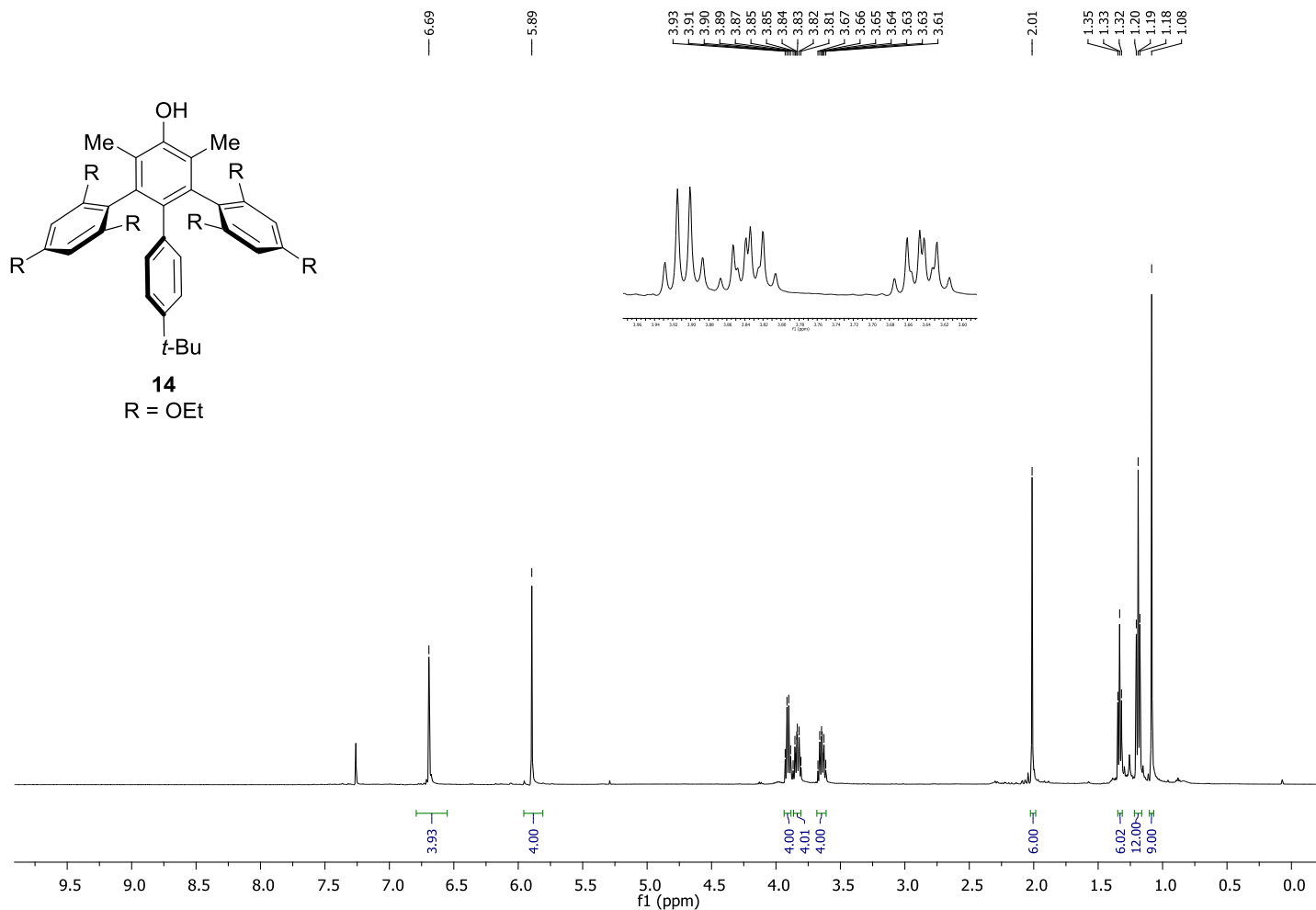

${ }^{13} \mathrm{C}$ NMR spectrum of compound $14\left(\mathrm{CDCl}_{3}, 125 \mathrm{MHz}\right)$

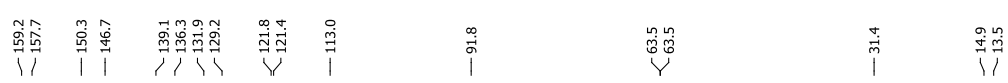
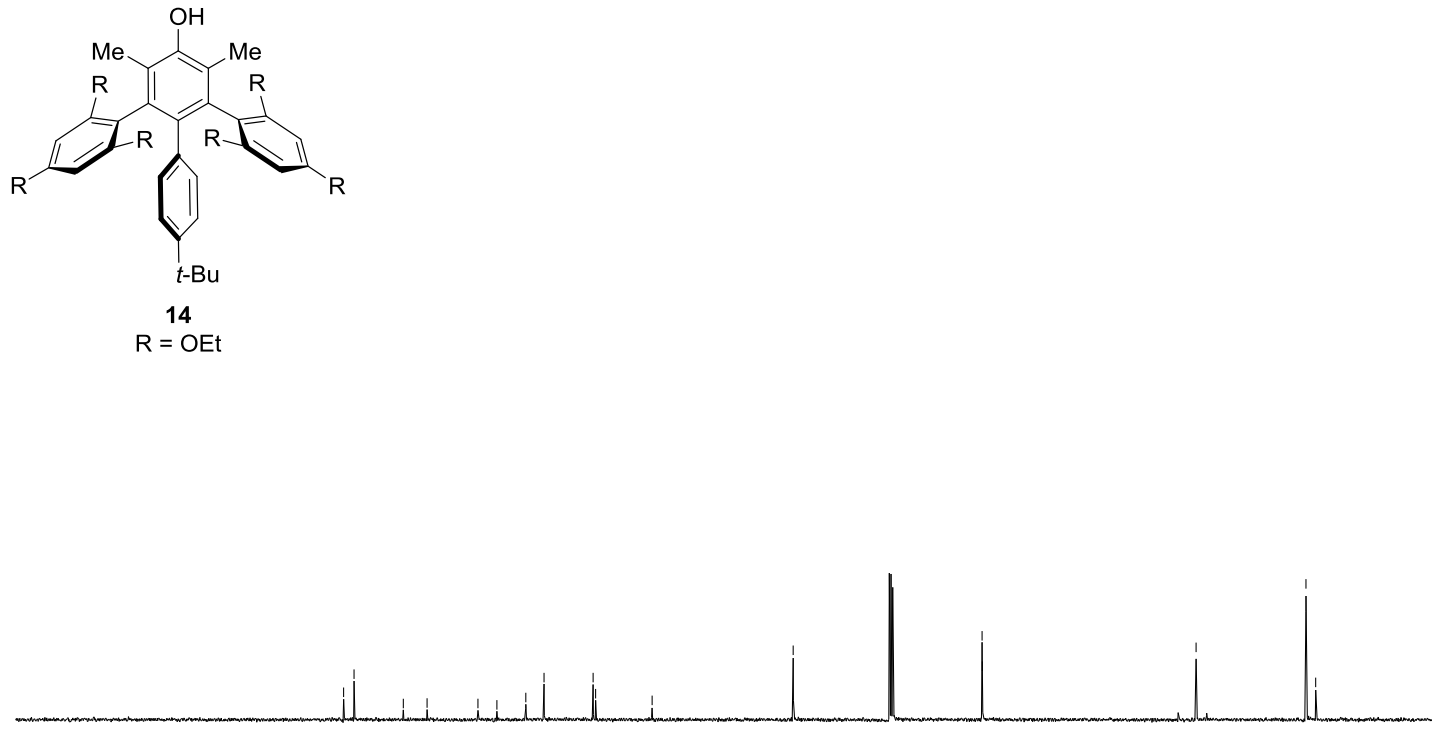

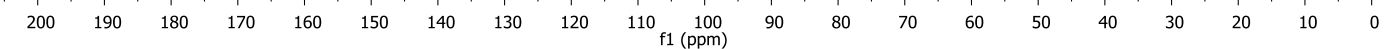


${ }^{1} \mathrm{H} \mathrm{NMR}$ spectrum of compound $15\left(\mathrm{CDCl}_{3}, 500 \mathrm{MHz}\right)$
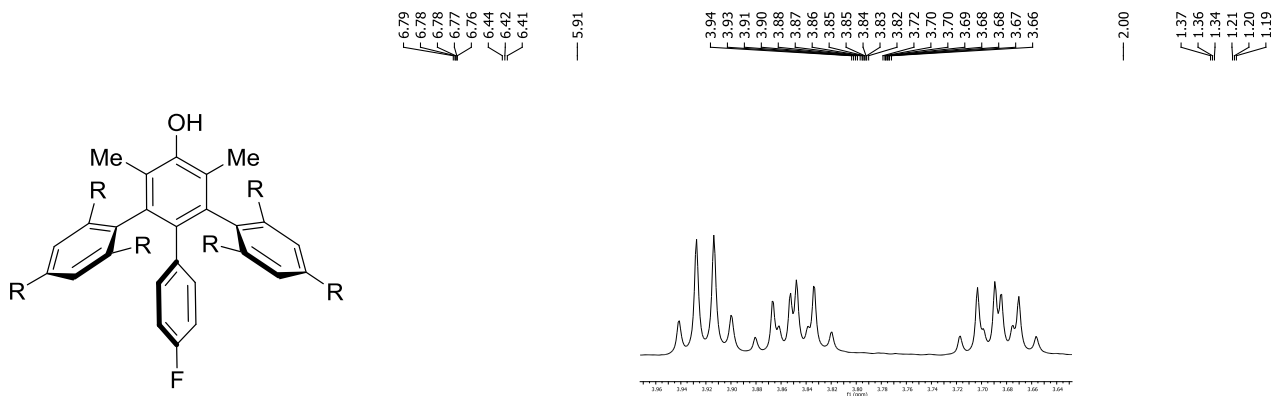

15
$R=O E$

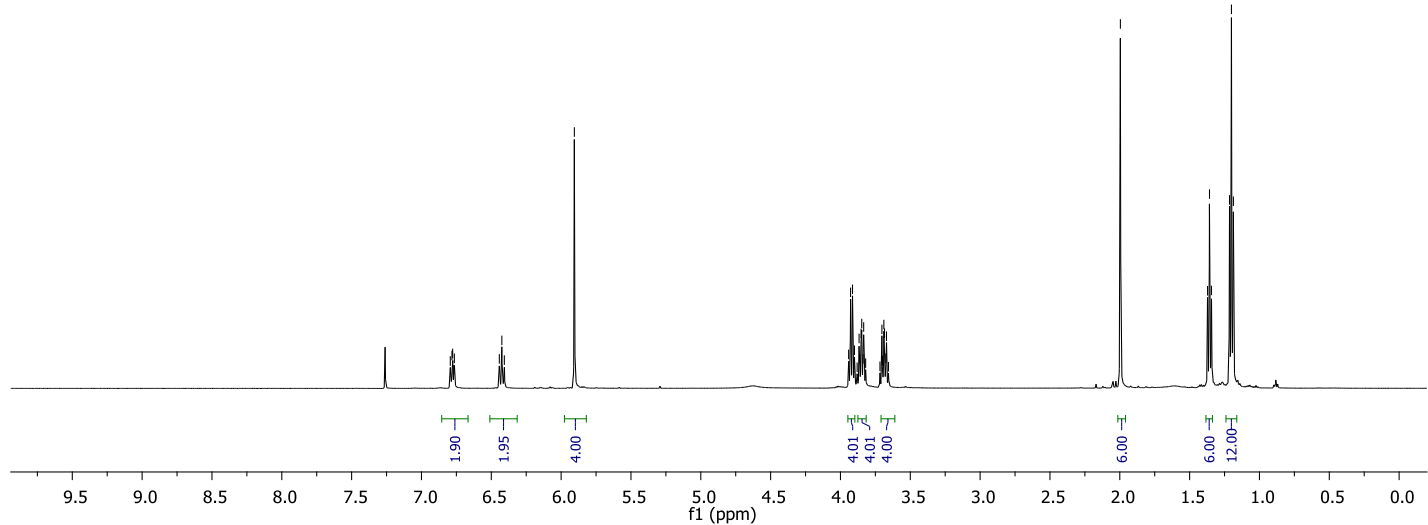

${ }^{13} \mathrm{C}$ NMR spectrum of compound $15\left(\mathrm{CDCl}_{3}, 125 \mathrm{MHz}\right)$
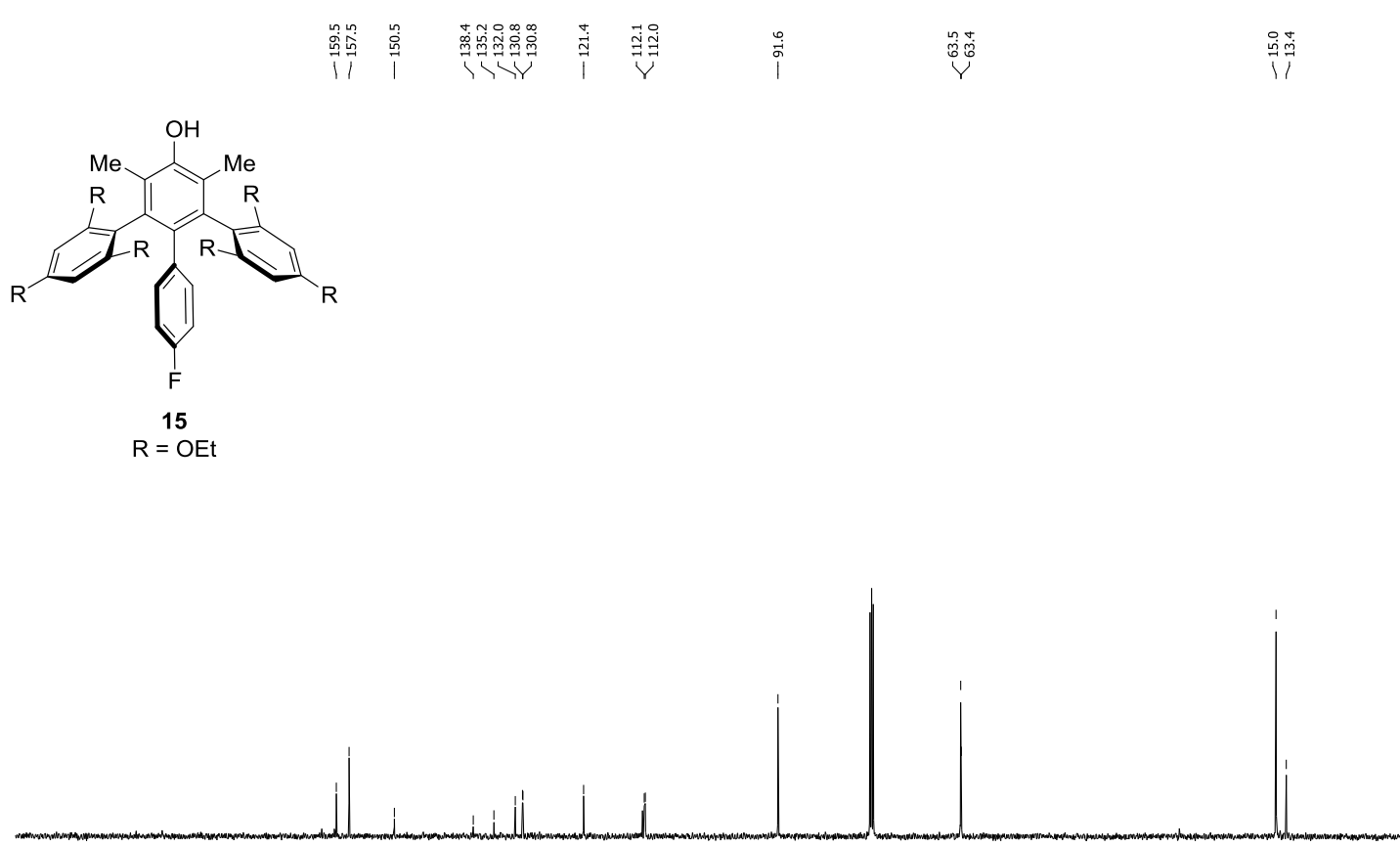

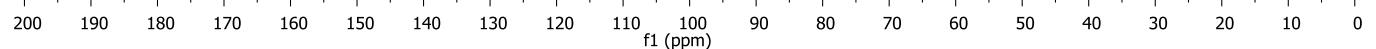


${ }^{19} \mathrm{~F}$ NMR spectrum of compound $15\left(\mathrm{CDCl}_{3}, 377 \mathrm{MHz}\right)$

$\stackrel{\substack{0 \\ \stackrel{g}{g}}}{i}$

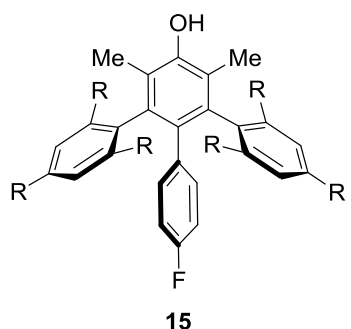

$\mathrm{R}=\mathrm{OEt}$

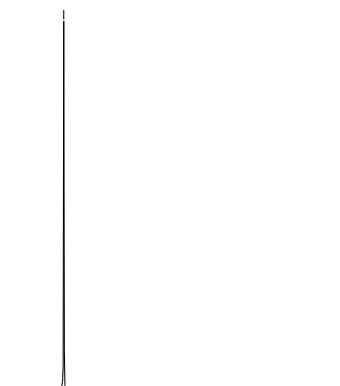

$\begin{array}{lllllllllllllllllllll}-119.5 & -120.0 & -120.5 & -121.0 & -121.5 & -122.0 & -122.5 & -123.0 & -123.5 & -124.0 & -124.5 & -125.0 & -125.5 & -126.0 & -126.5 & -127.0 & -127.5 & -128.0 & -128.5 & -129.0 & -129\end{array}$ 
${ }^{1} \mathrm{H}$ NMR spectrum of compound $16\left(\mathrm{CDCl}_{3}, 400 \mathrm{MHz}\right)$

可

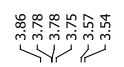

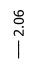
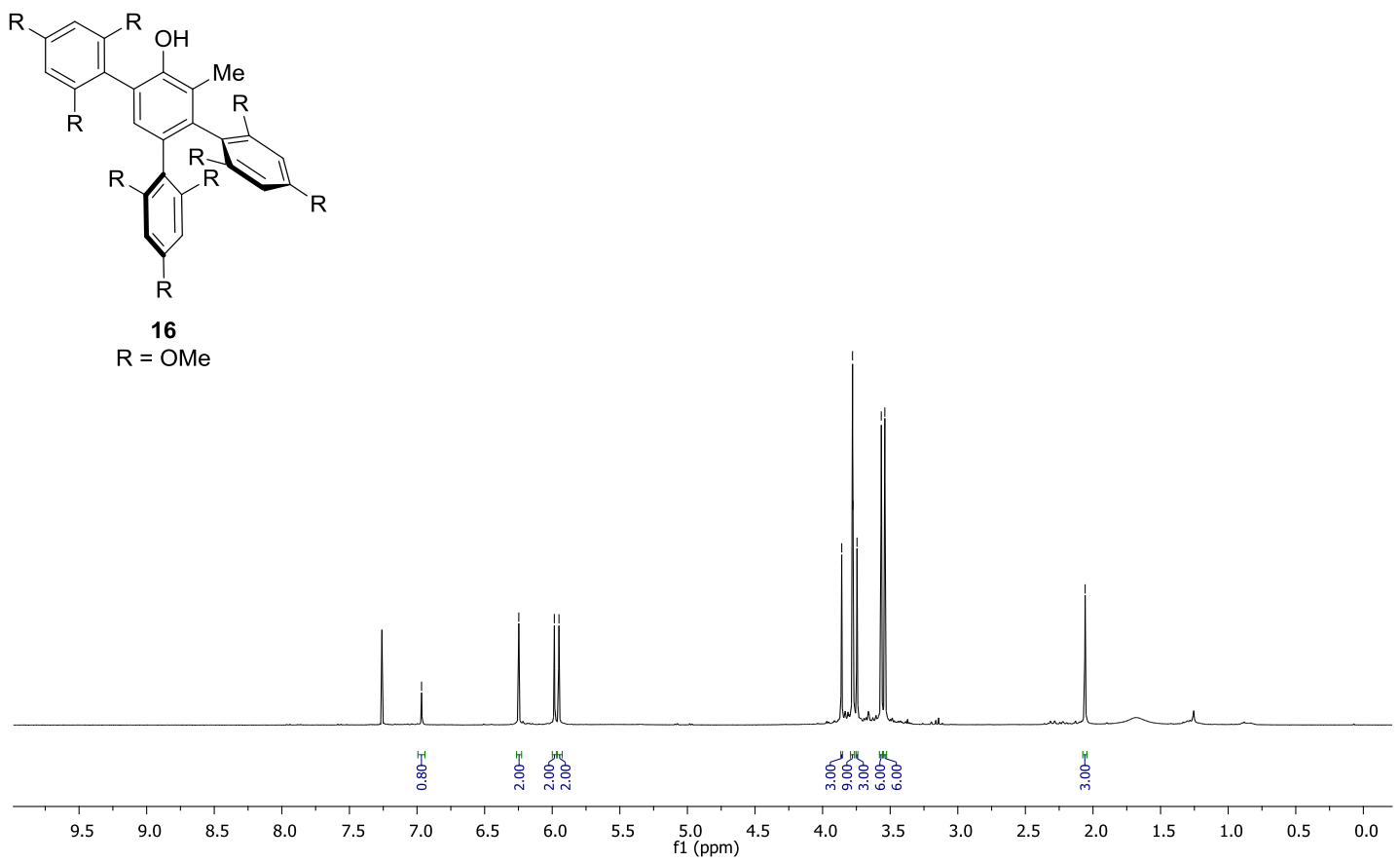

${ }^{13} \mathrm{C}$ NMR spectrum of compound $16\left(\mathrm{CDCl}_{3}, 100 \mathrm{MHz}\right)$

ㅁำ

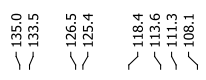

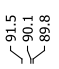

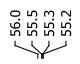

$\underset{+}{i}$
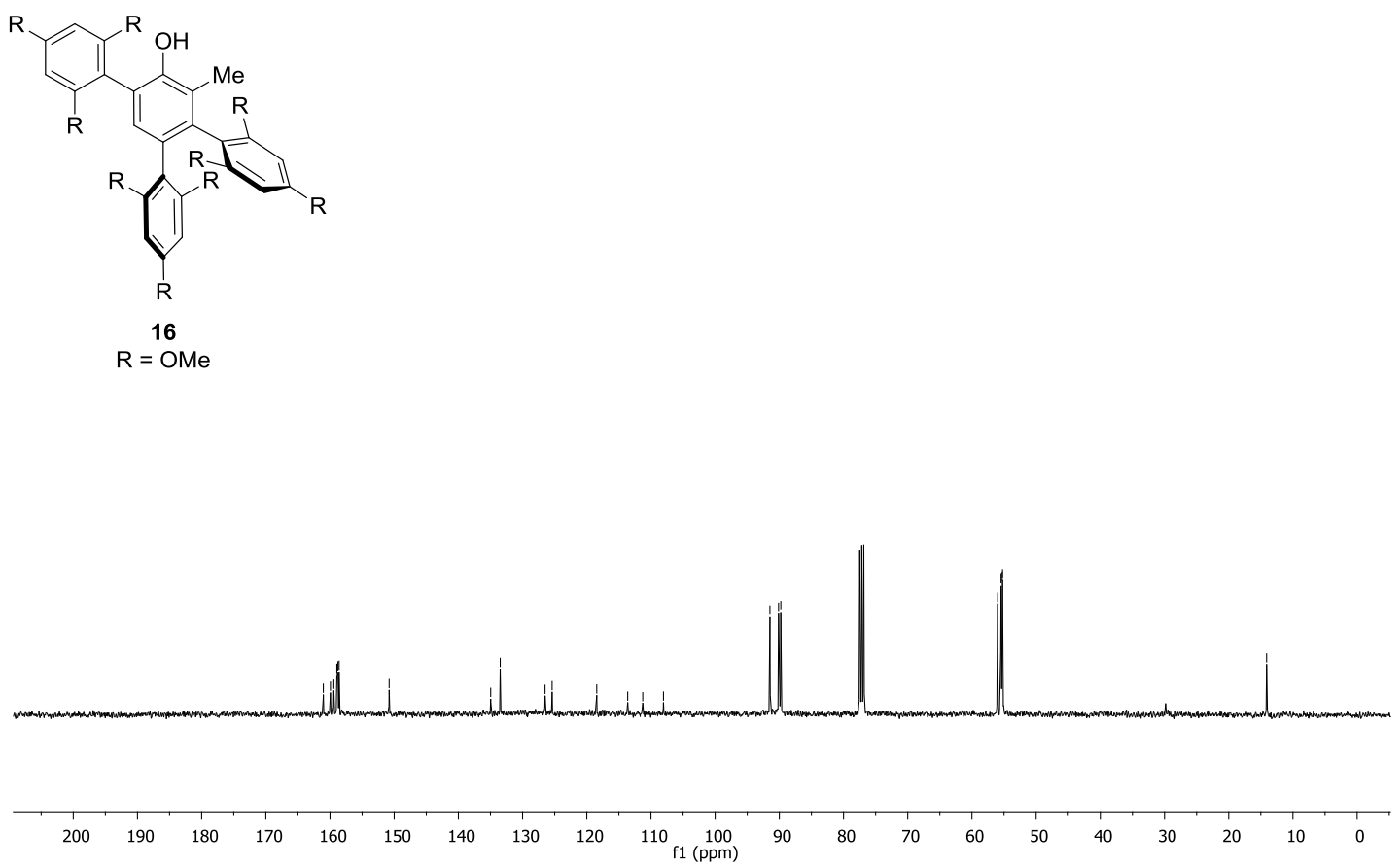

S46 
${ }^{1} \mathrm{H}$ NMR spectrum of compound $17\left(\mathrm{CDCl}_{3}, 500 \mathrm{MHz}\right)$

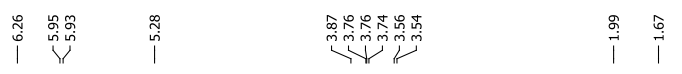
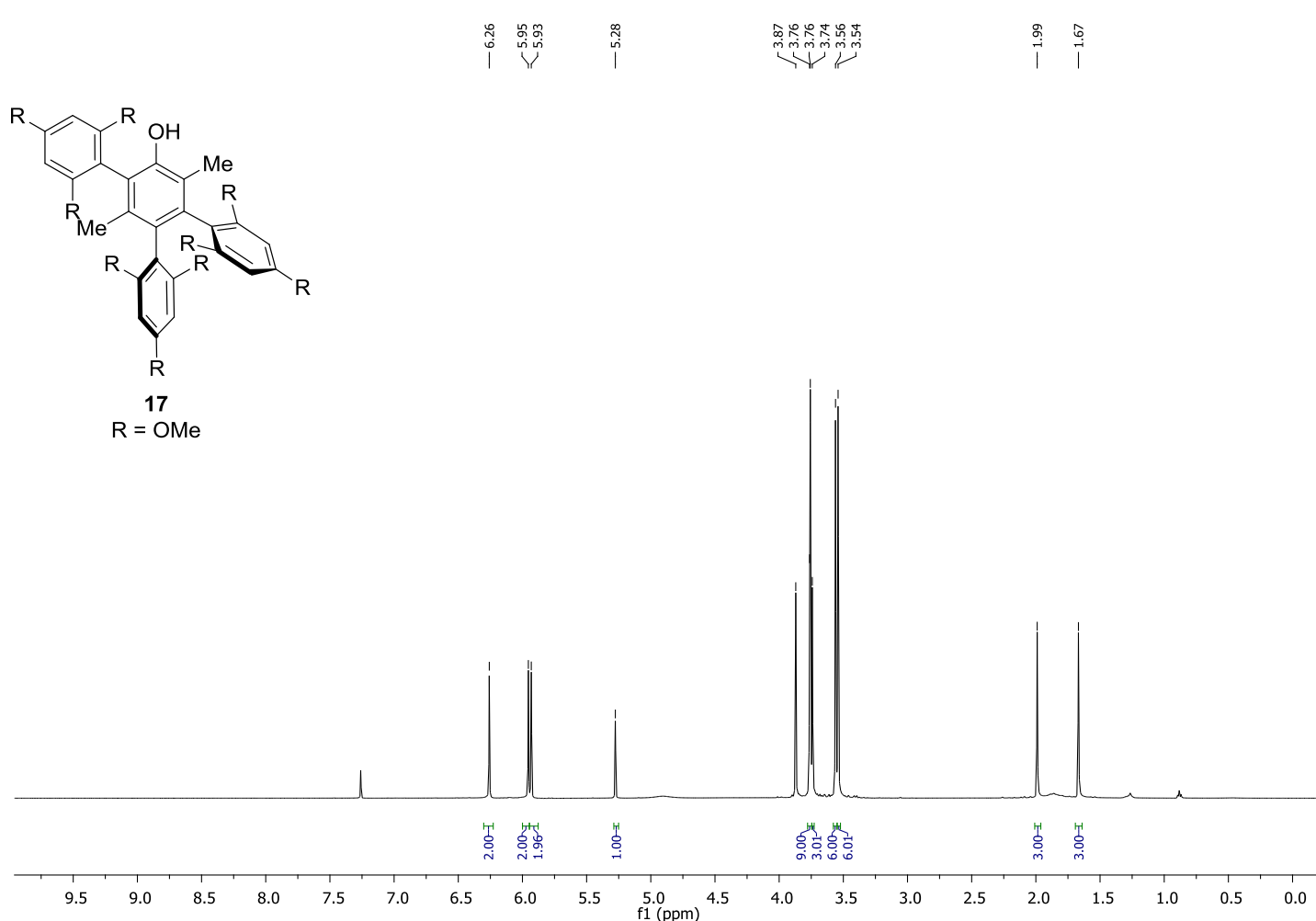

${ }^{13} \mathrm{C}$ NMR spectrum of compound $17\left(\mathrm{CDCl}_{3}, 125 \mathrm{MHz}\right)$

\begin{tabular}{|c|c|c|c|}
\hline 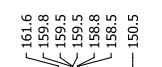 & 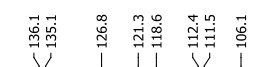 & 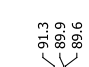 & 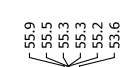 \\
\hline
\end{tabular}

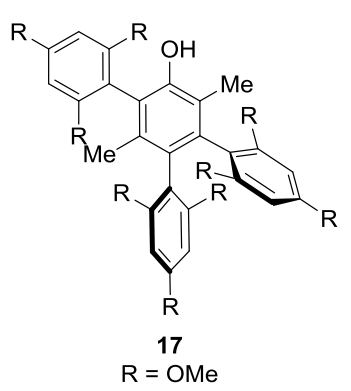

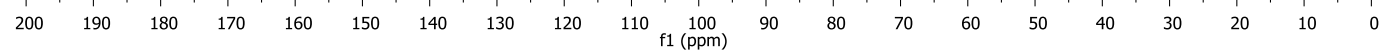


${ }^{1} \mathrm{H}$ NMR spectrum of compound $18\left(\mathrm{CDCl}_{3}, 400 \mathrm{MHz}\right)$

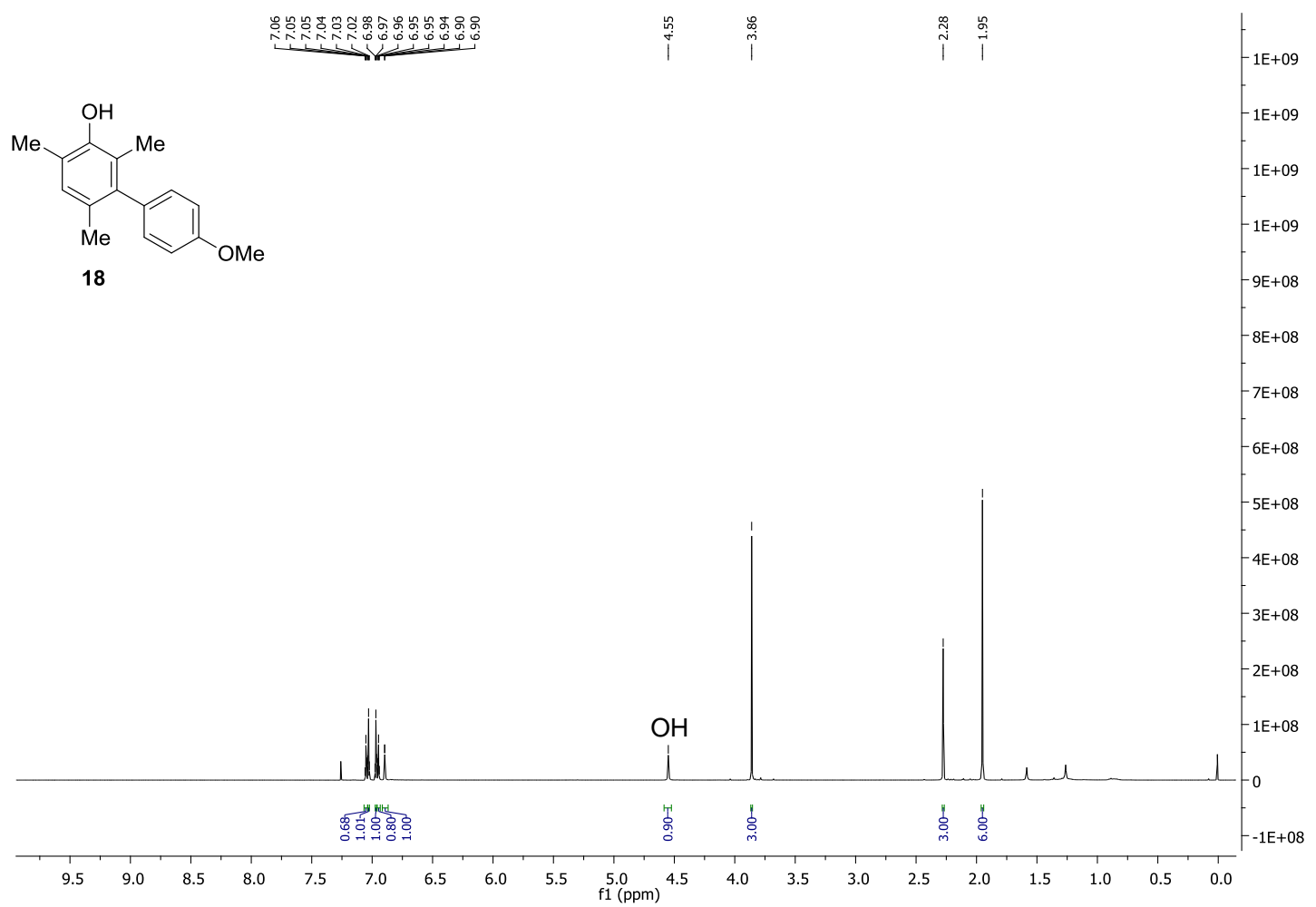

${ }^{13} \mathrm{C}$ NMR spectrum of compound $\mathbf{1 8}\left(\mathrm{CDCl}_{3}, 100 \mathrm{MHz}\right)$

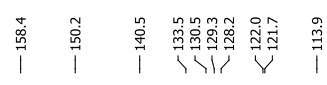
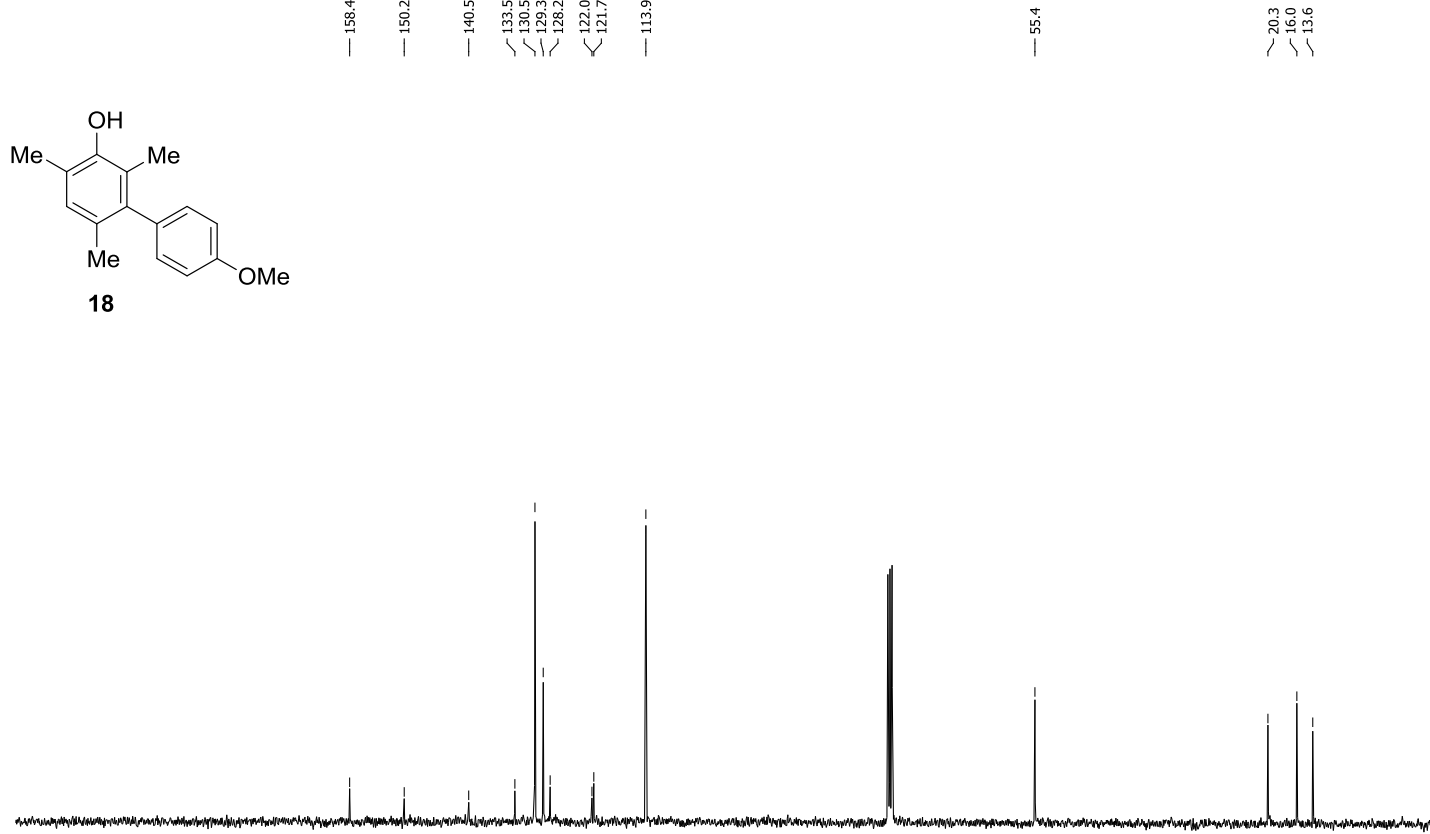

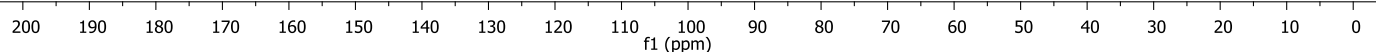


${ }^{1} \mathrm{H}$ NMR spectrum of compound $19\left(\mathrm{CDCl}_{3}, 400 \mathrm{MHz}\right)$
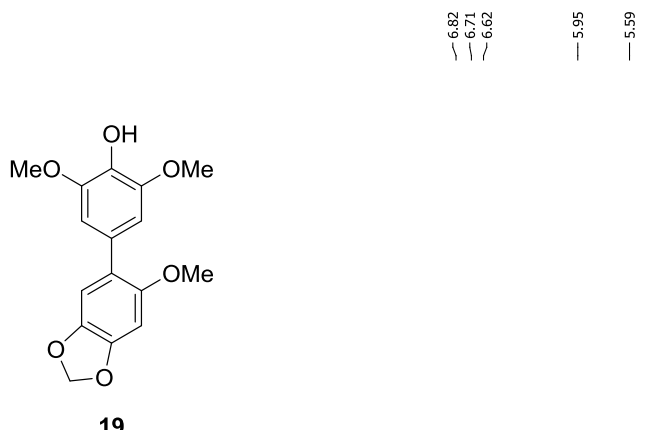

19

$\mathrm{OH}$

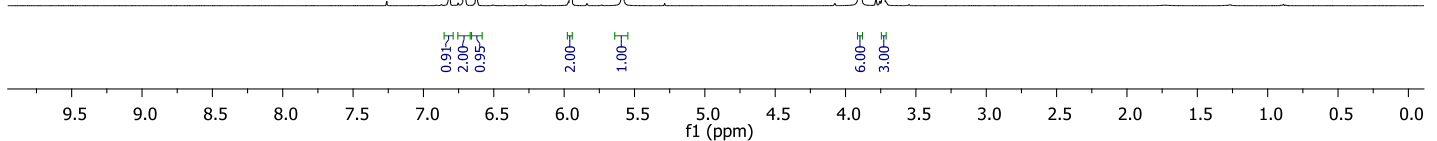

${ }^{13} \mathrm{C}$ NMR spectrum of compound $19\left(\mathrm{CDCl}_{3}, 100 \mathrm{MHz}\right)$
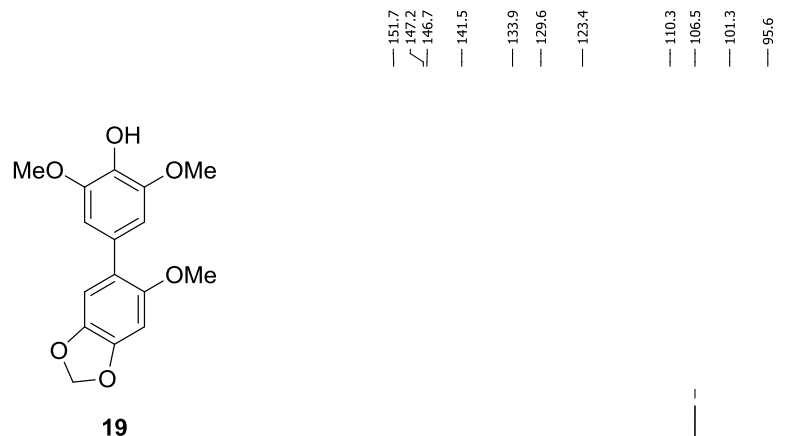

19

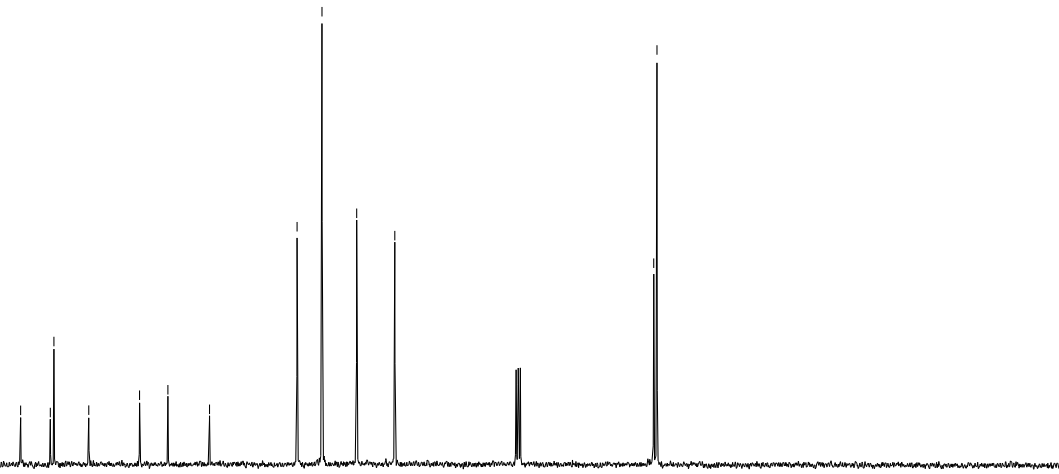

$\begin{array}{llllllllll}200 & 190 & 180 & 170 & 160 & 150 & 140 & 130 & 120 & 110 \\ \mathrm{f} 1(\mathrm{ppm})\end{array}$ 
${ }^{1} \mathrm{H}$ NMR spectrum of compound $20\left(\mathrm{CDCl}_{3}, 400 \mathrm{MHz}\right)$
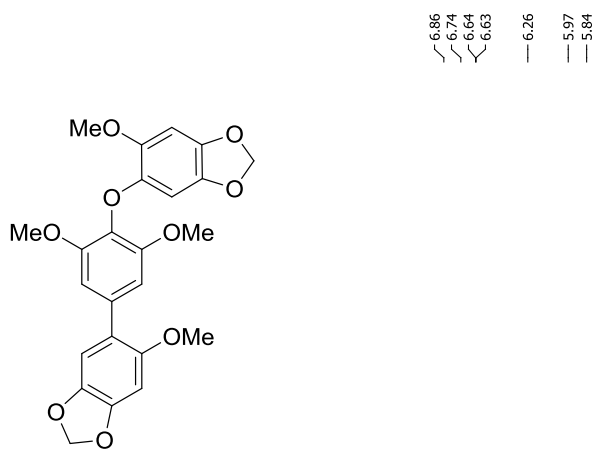

20

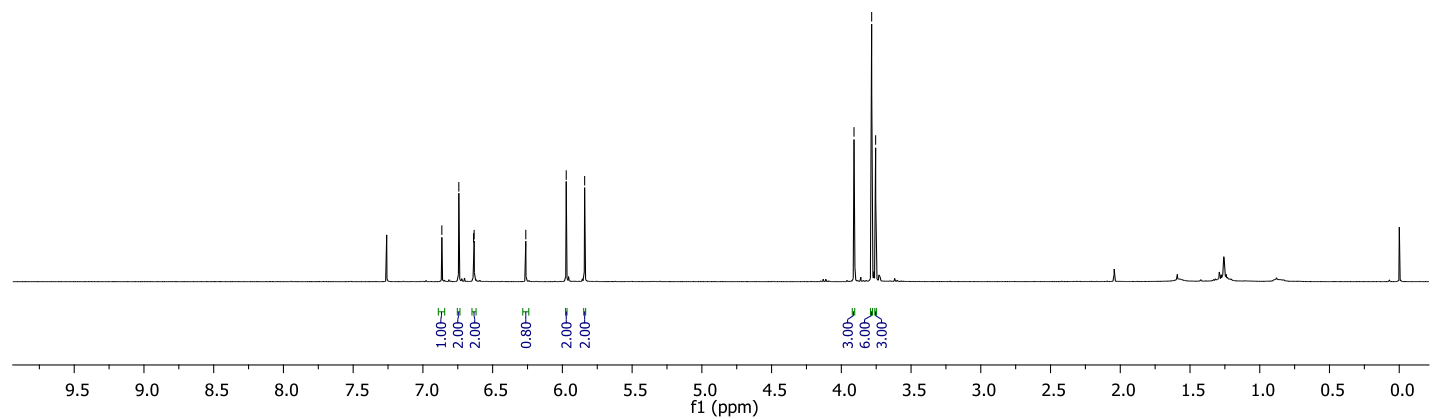

${ }^{13} \mathrm{C}$ NMR spectrum of compound $20\left(\mathrm{CDCl}_{3}, 100 \mathrm{MHz}\right)$

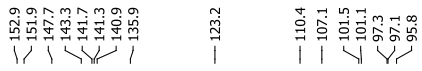
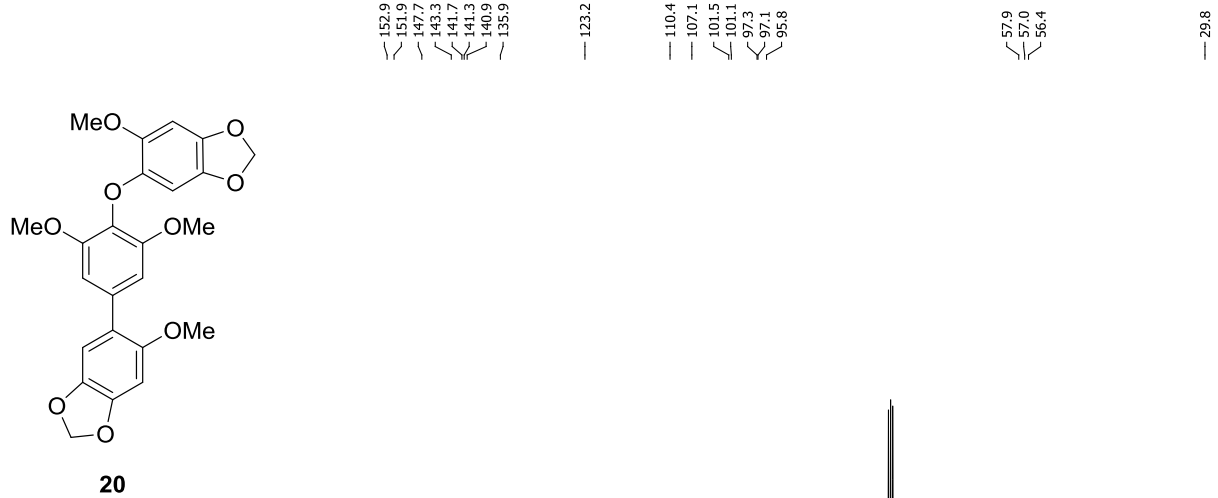

20

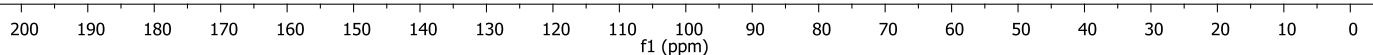


${ }^{1} \mathrm{H}$ NMR spectrum of compound $21\left(\mathrm{CDCl}_{3}, 400 \mathrm{MHz}\right)$
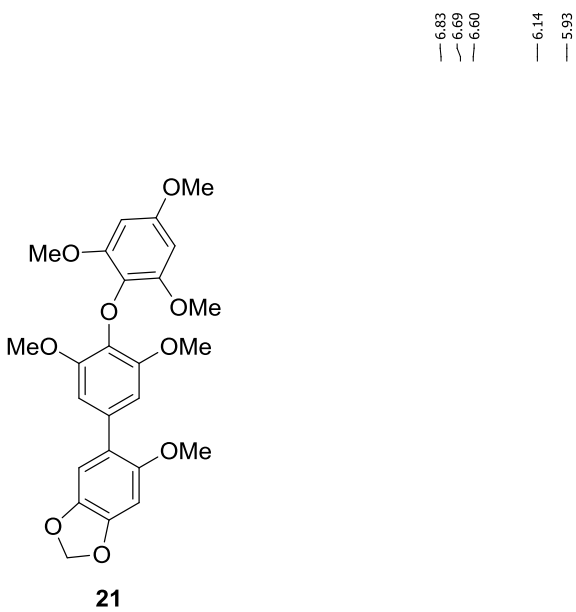

)

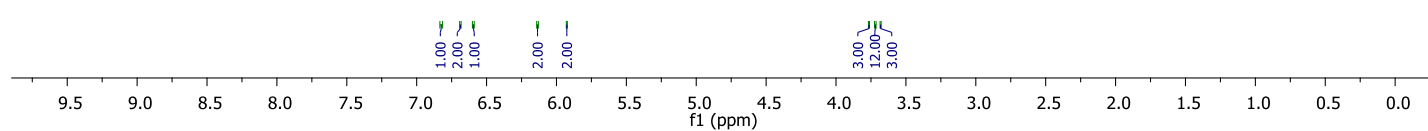

${ }^{13} \mathrm{C}$ NMR spectrum of compound $21\left(\mathrm{CDCl}_{3}, 100 \mathrm{MHz}\right)$

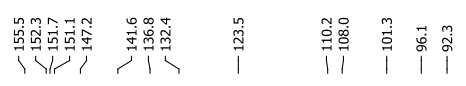

y象
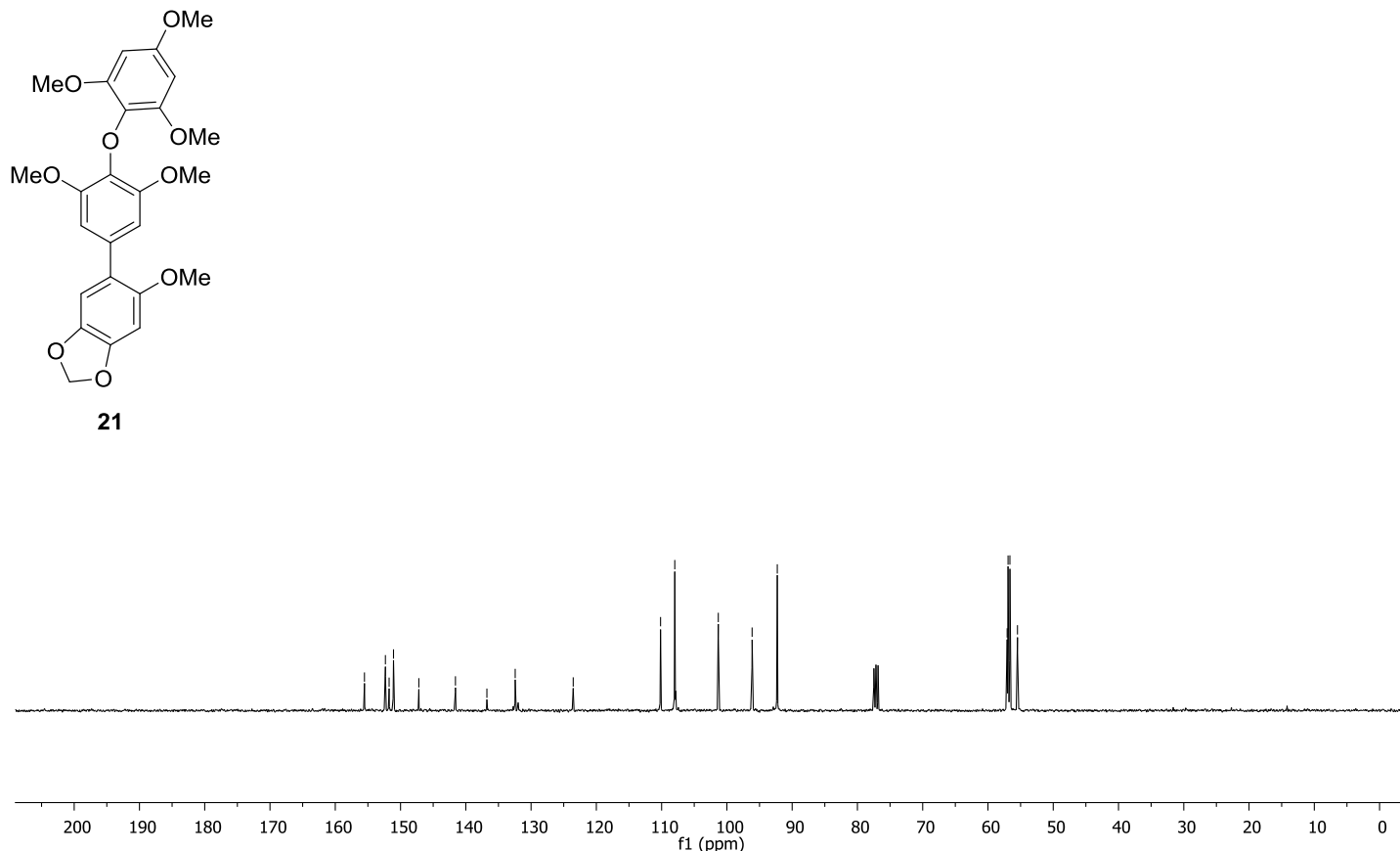
${ }^{1} \mathrm{H}$ NMR spectrum of compound $22\left(\mathrm{CDCl}_{3}, 400 \mathrm{MHz}\right)$

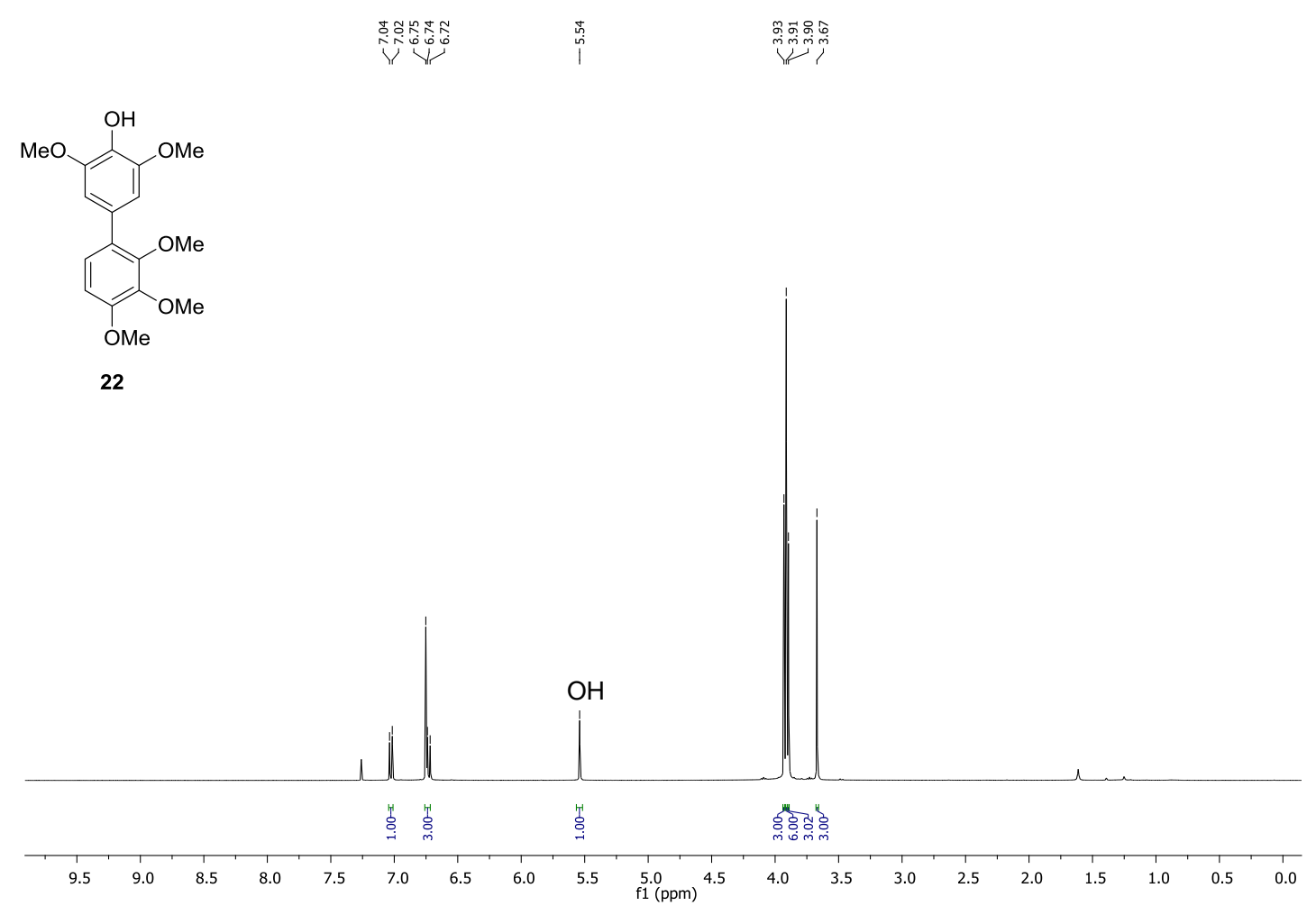

${ }^{13} \mathrm{C}$ NMR spectrum of compound $22\left(\mathrm{CDCl}_{3}, 100 \mathrm{MHz}\right)$
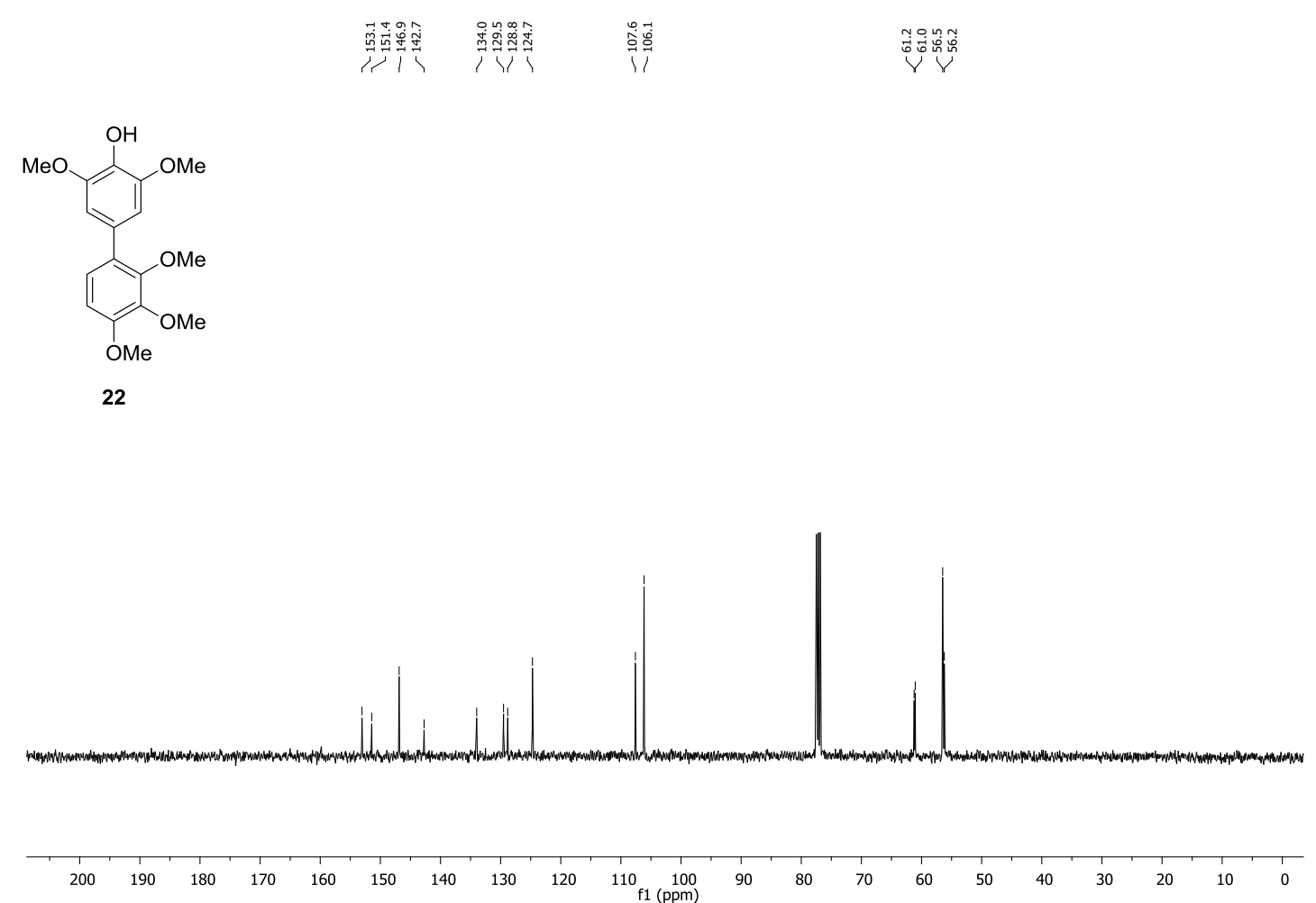
${ }^{1} \mathrm{H}$ NMR spectrum of compound $23\left(\mathrm{CDCl}_{3}, 400 \mathrm{MHz}\right)$

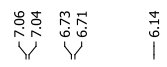

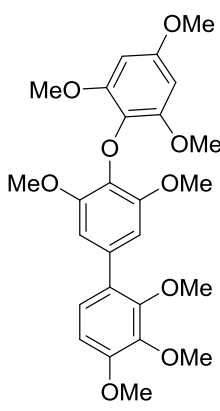

23

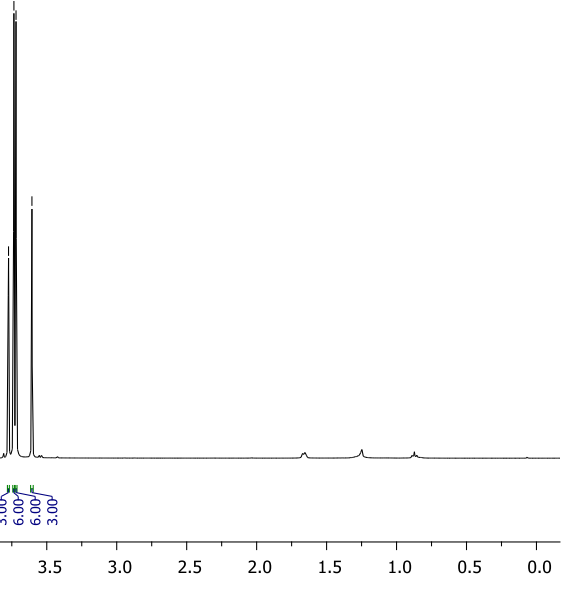

${ }^{13} \mathrm{C}$ NMR spectrum of compound $23\left(\mathrm{CDCl}_{3}, 100 \mathrm{MHz}\right)$
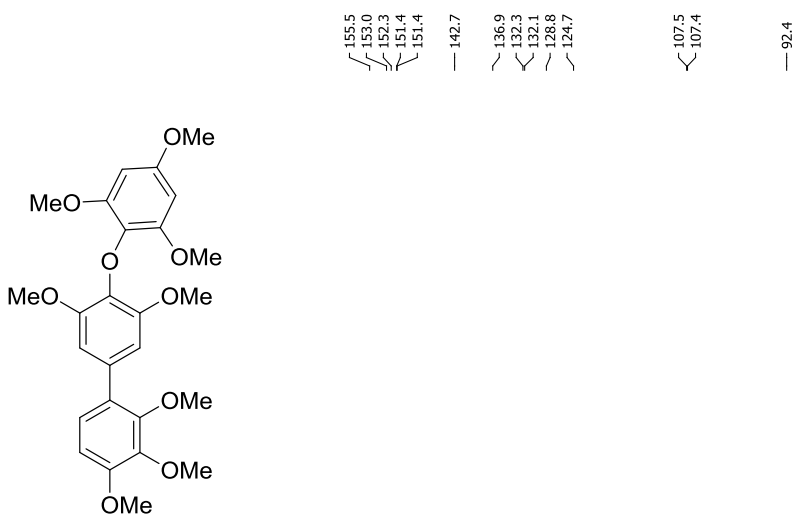

23

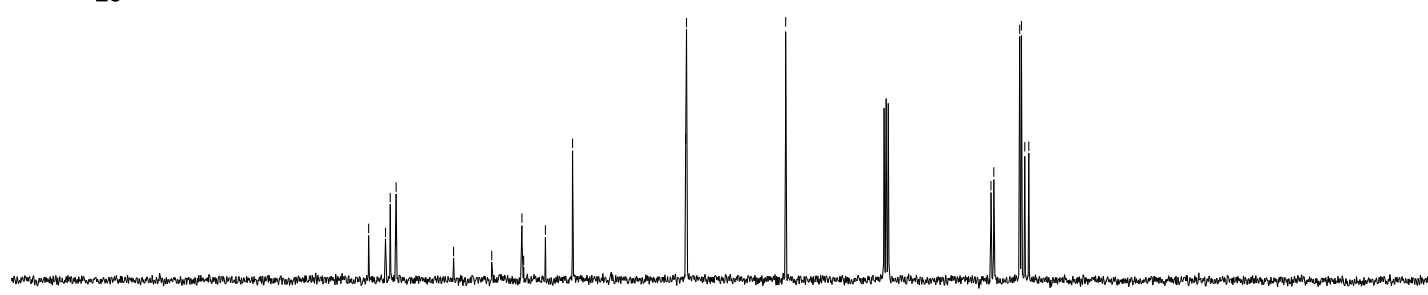

$\begin{array}{lllllllllllllllllllll}200 & 190 & 180 & 170 & 160 & 150 & 140 & 130 & 120 & 110 & 100 & 90 & 80 & 70 & 60 & 50 & 40 & 30 & 20 & 10 & 0\end{array}$ 
${ }^{1} \mathrm{H}$ NMR spectrum of compound $24\left(\mathrm{CDCl}_{3}, 400 \mathrm{MHz}\right)$
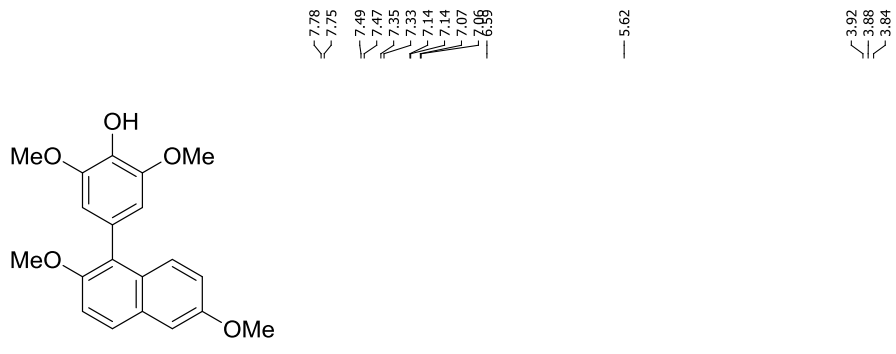

24

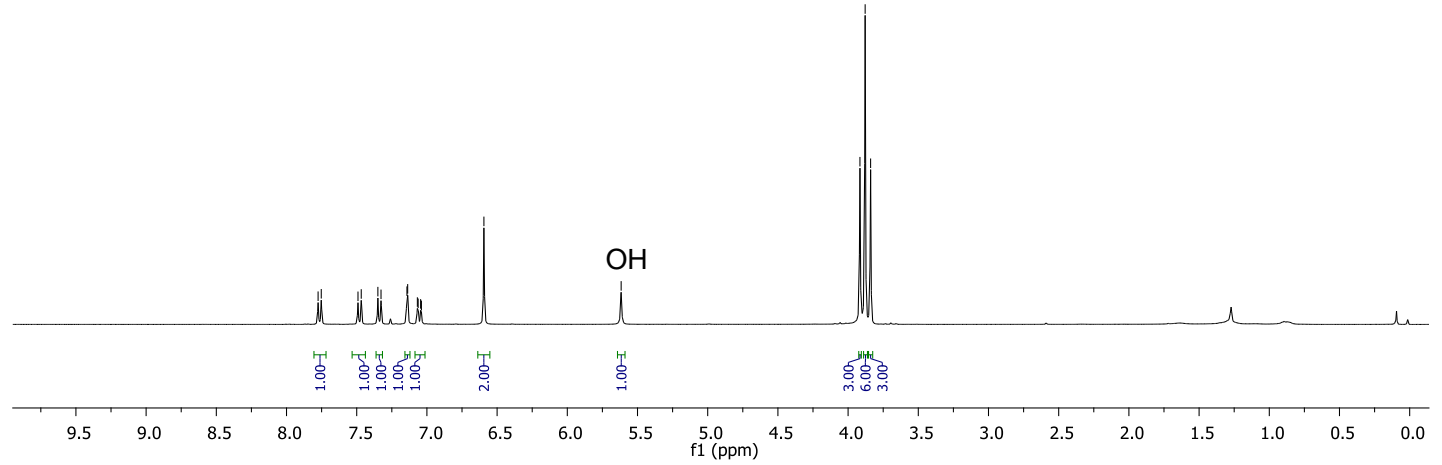

${ }^{13} \mathrm{C}$ NMR spectrum of compound $24\left(\mathrm{CDCl}_{3}, 100 \mathrm{MHz}\right)$
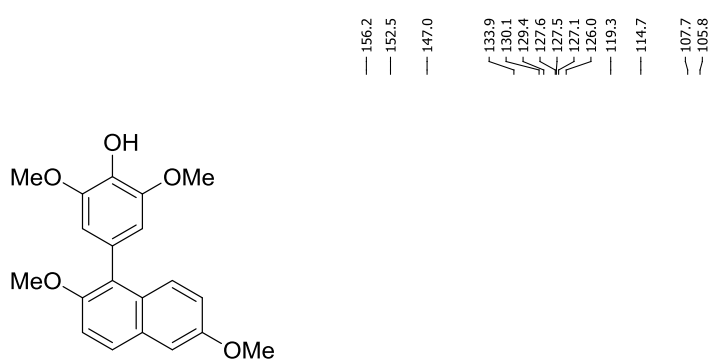

24
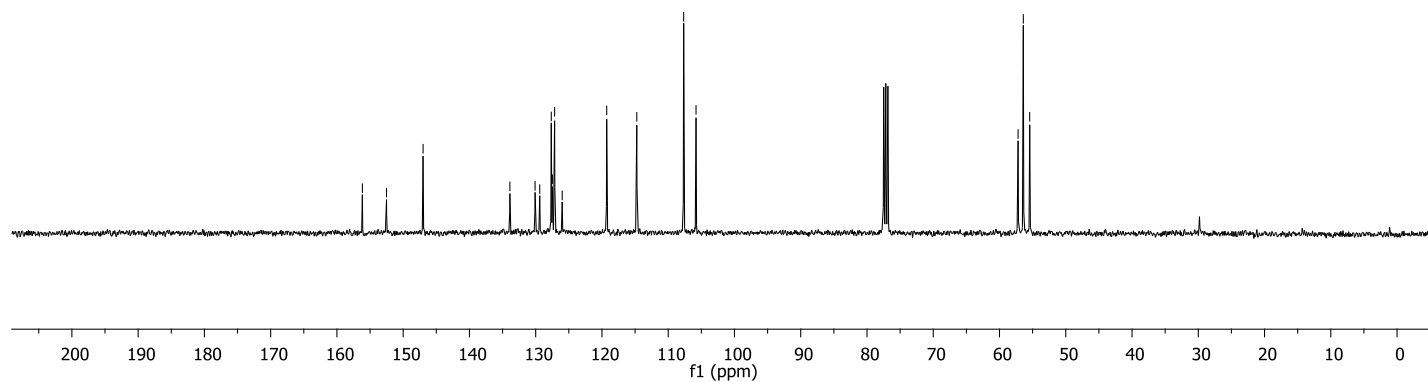
${ }^{1} \mathrm{H}$ NMR spectrum of compound $25\left(\mathrm{CDCl}_{3}, 400 \mathrm{MHz}\right)$

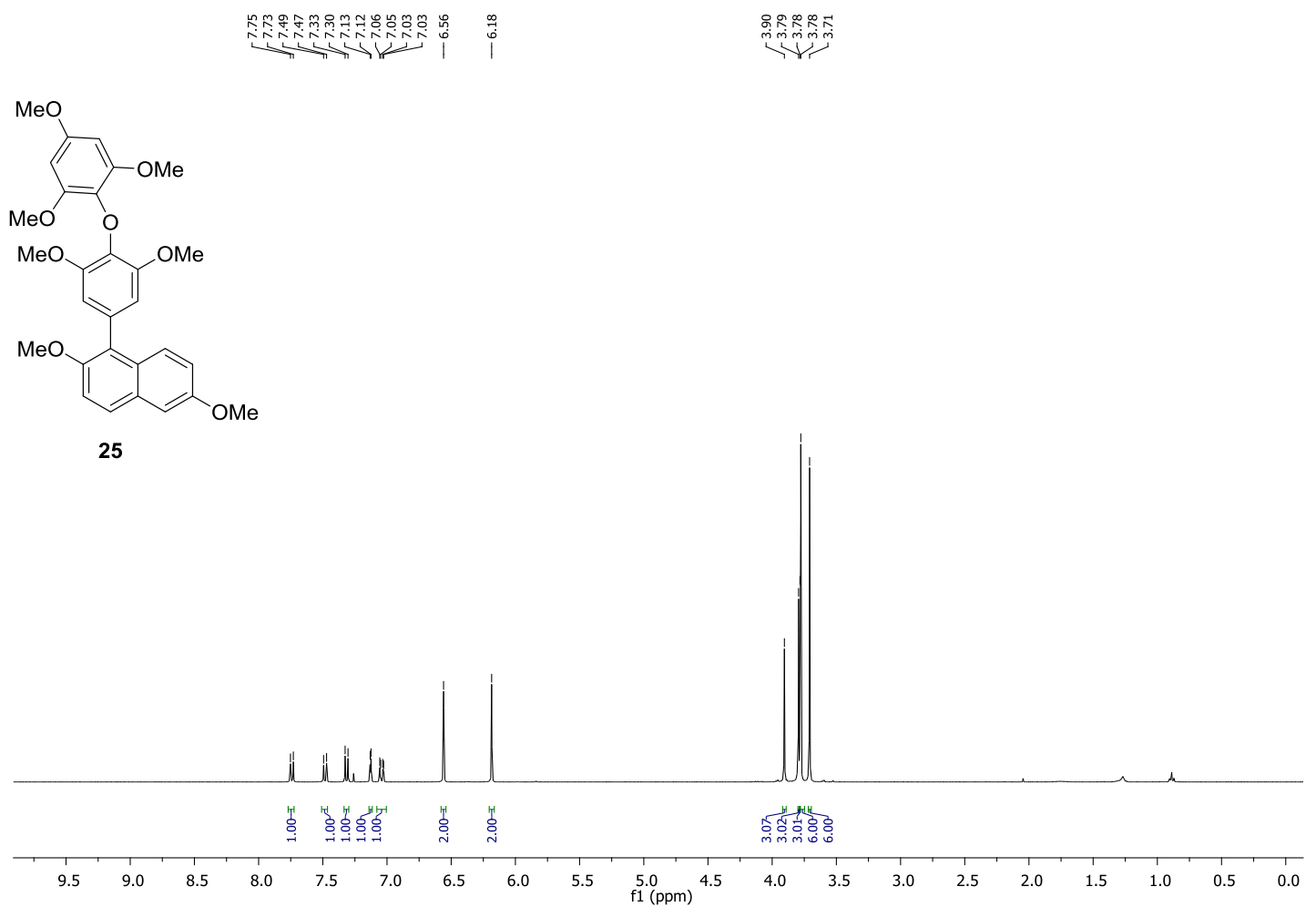

${ }^{13} \mathrm{C}$ NMR spectrum of compound $25\left(\mathrm{CDCl}_{3}, 100 \mathrm{MHz}\right)$

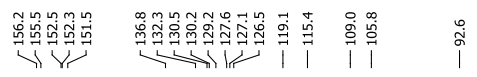

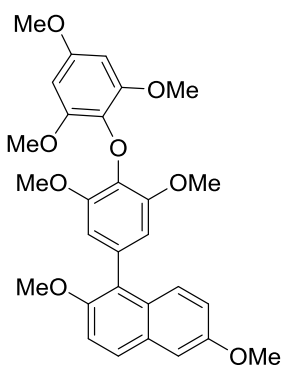

25

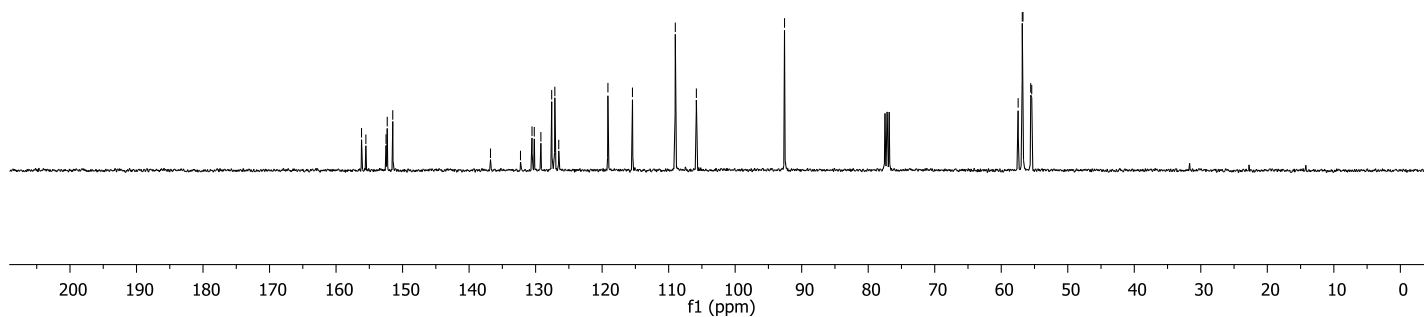


${ }^{1} \mathrm{H}$ NMR spectrum of compound $26\left(\mathrm{CDCl}_{3}, 400 \mathrm{MHz}\right)$

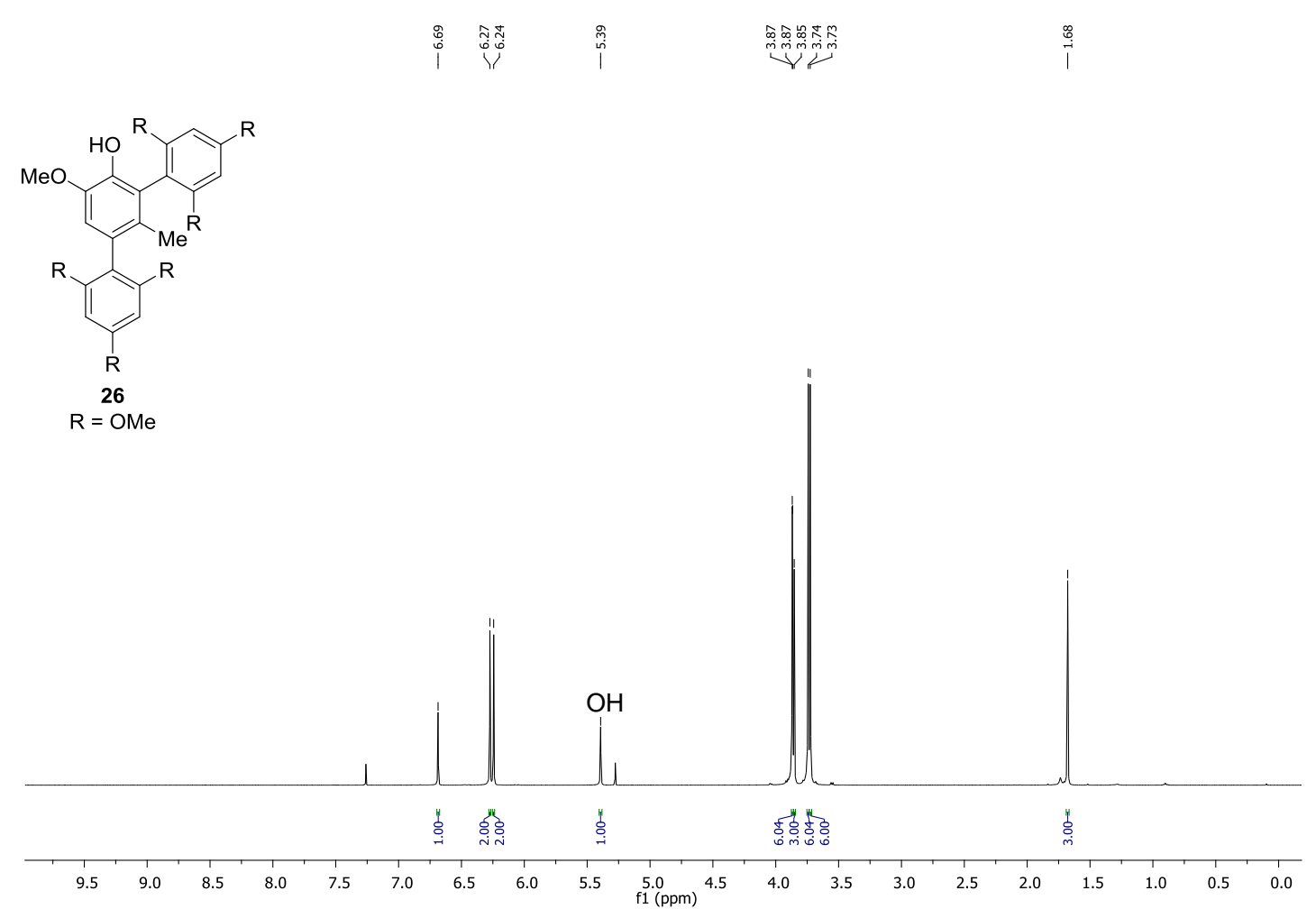

${ }^{13} \mathrm{C}$ NMR spectrum of compound $26\left(\mathrm{CDCl}_{3}, 100 \mathrm{MHz}\right)$
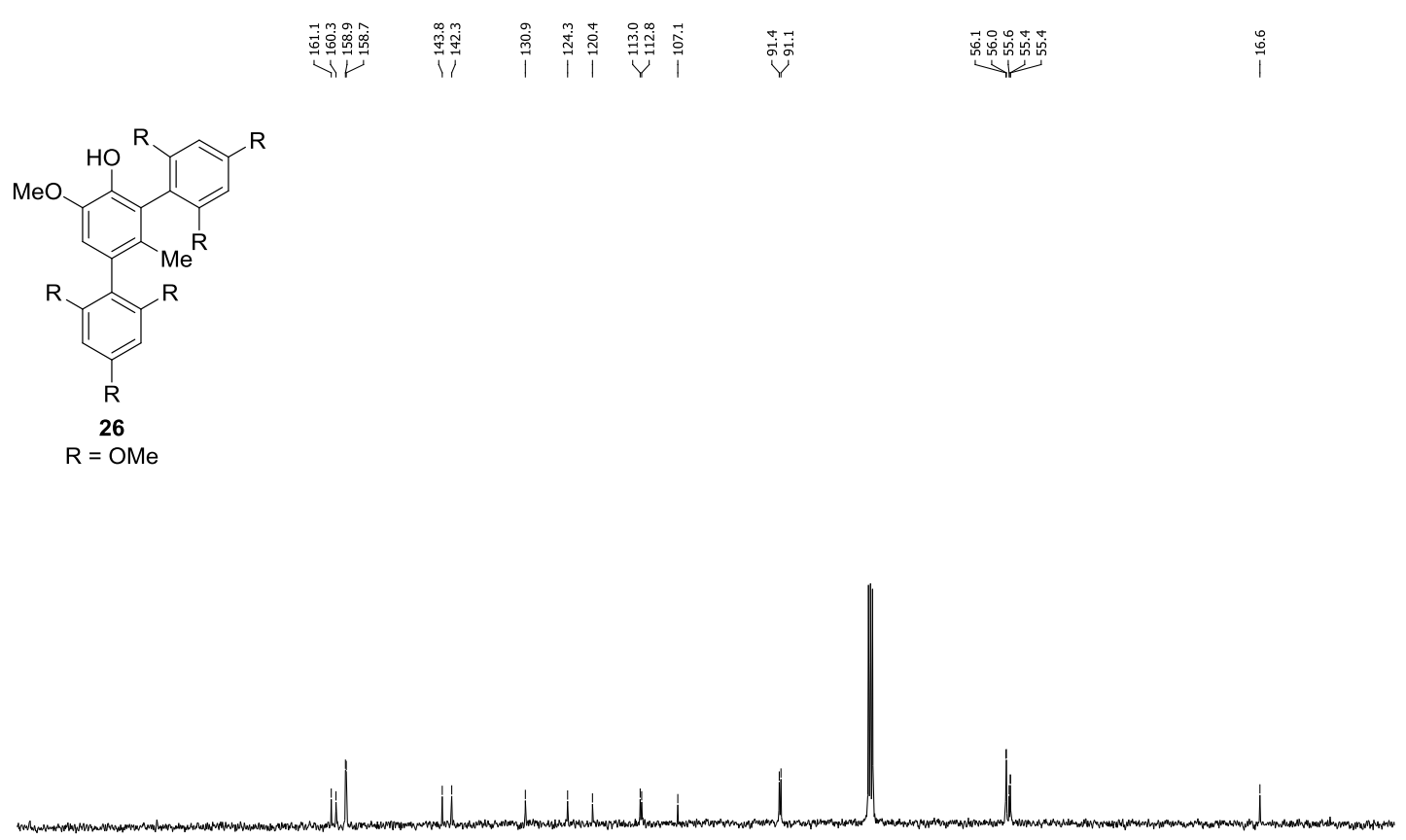

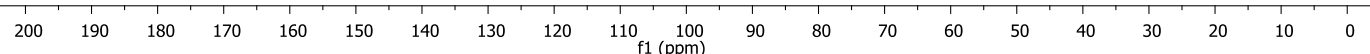


${ }^{1} \mathrm{H}$ NMR spectrum of compound 28a $\left(\mathrm{CDCl}_{3}, 400 \mathrm{MHz}\right)$

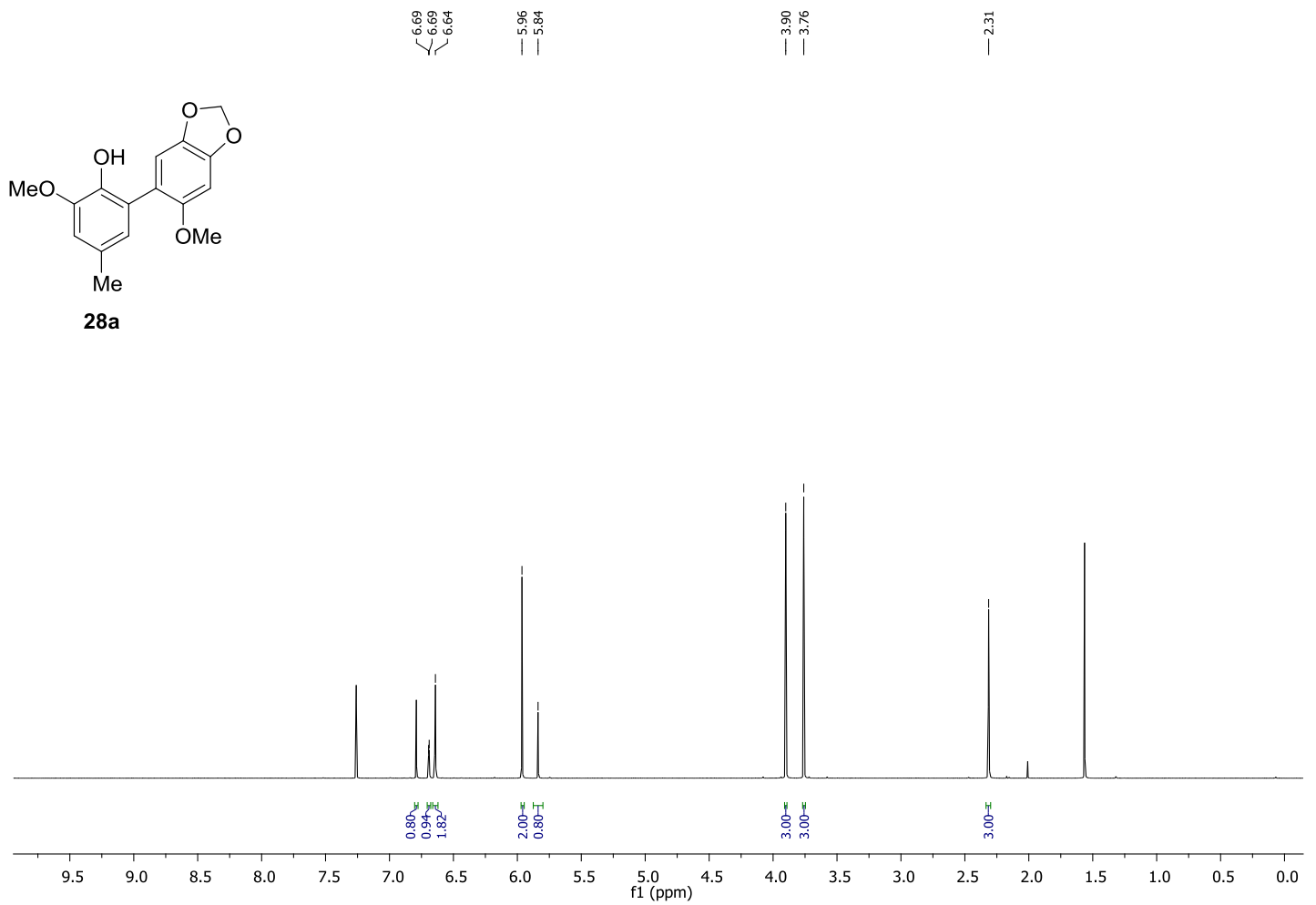

${ }^{13} \mathrm{C}$ NMR spectrum of compound $\mathbf{2 8 a}\left(\mathrm{CDCl}_{3}, 100 \mathrm{MHz}\right)$

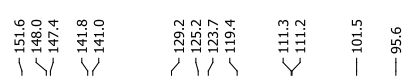

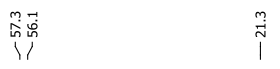

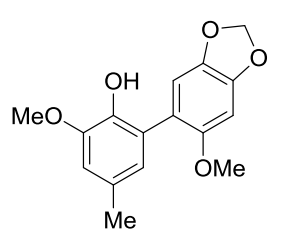

28a
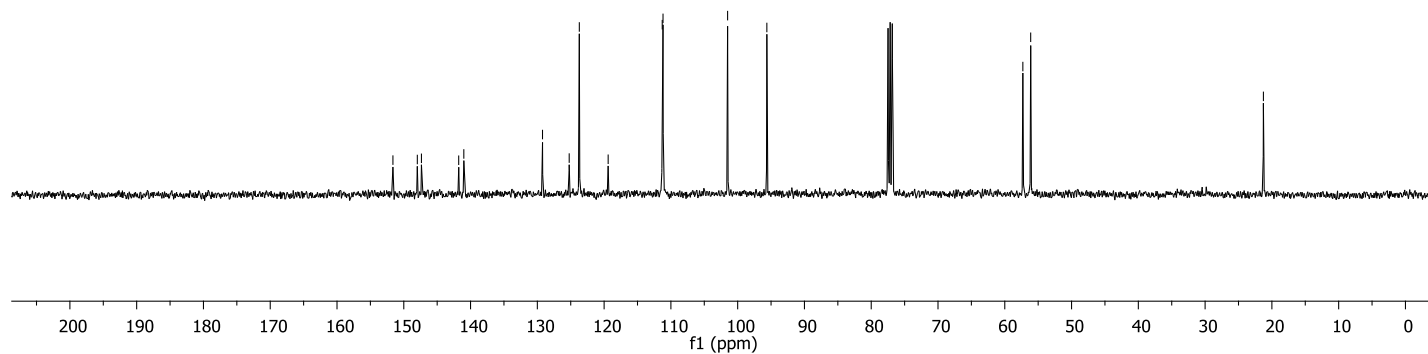
${ }^{1} \mathrm{H}$ NMR spectrum of compound $\mathbf{2 8 b}\left(\mathrm{CDCl}_{3}, 400 \mathrm{MHz}\right)$

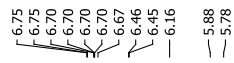

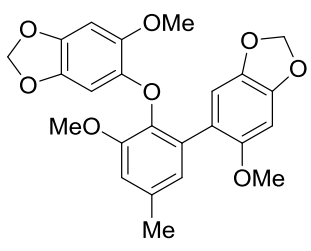

28b

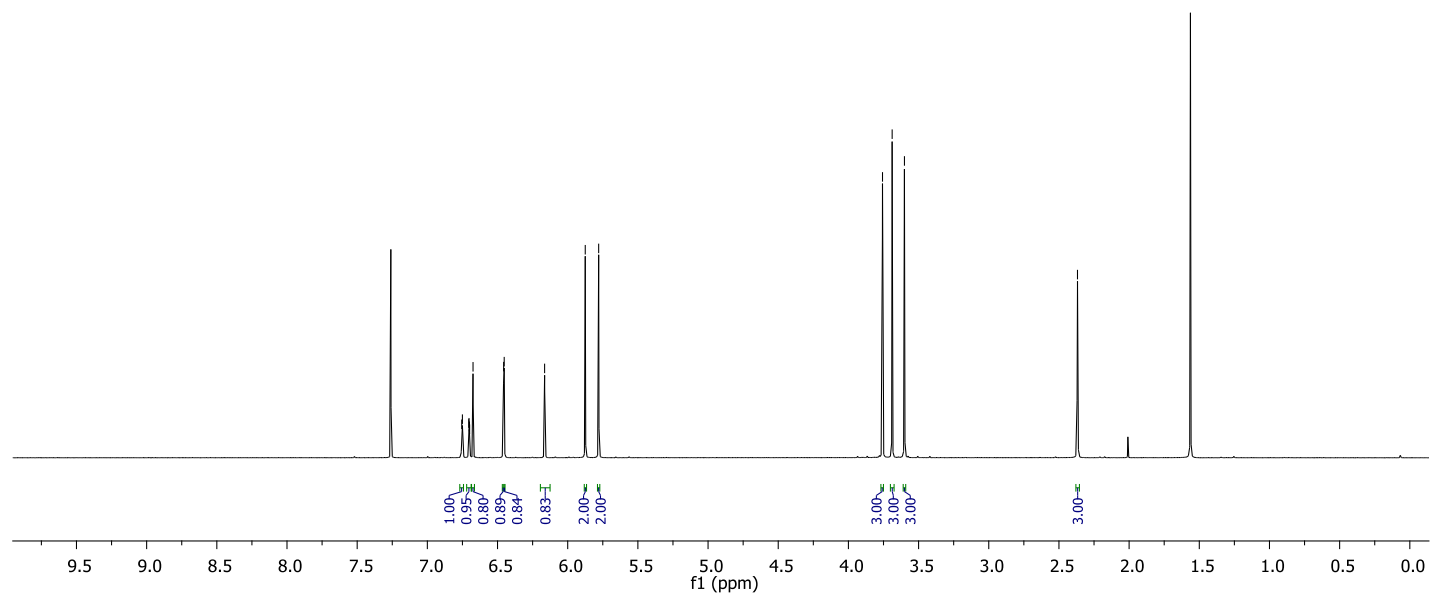

${ }^{13} \mathrm{C}$ NMR spectrum of compound $\mathbf{2 8 b}\left(\mathrm{CDCl}_{3}, 100 \mathrm{MHz}\right)$

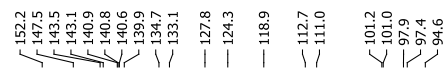

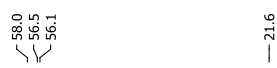

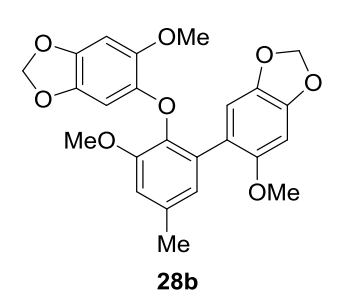

28b

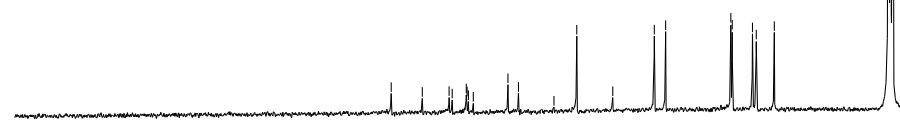
$\stackrel{9}{i}$
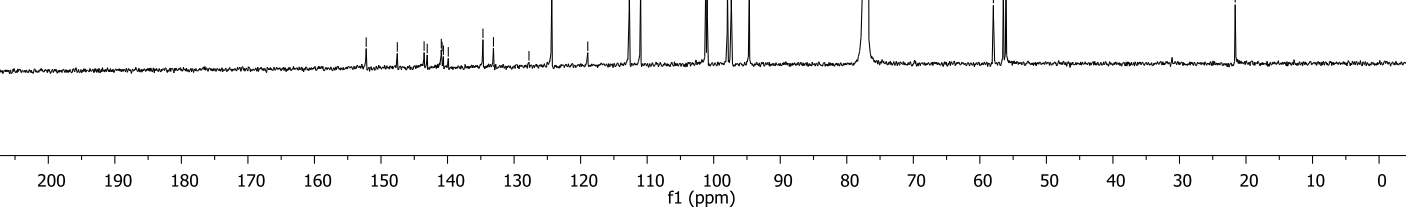
${ }^{1} \mathrm{H}$ NMR spectrum of compound $29\left(\mathrm{CDCl}_{3}, 500 \mathrm{MHz}\right)$

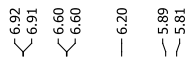
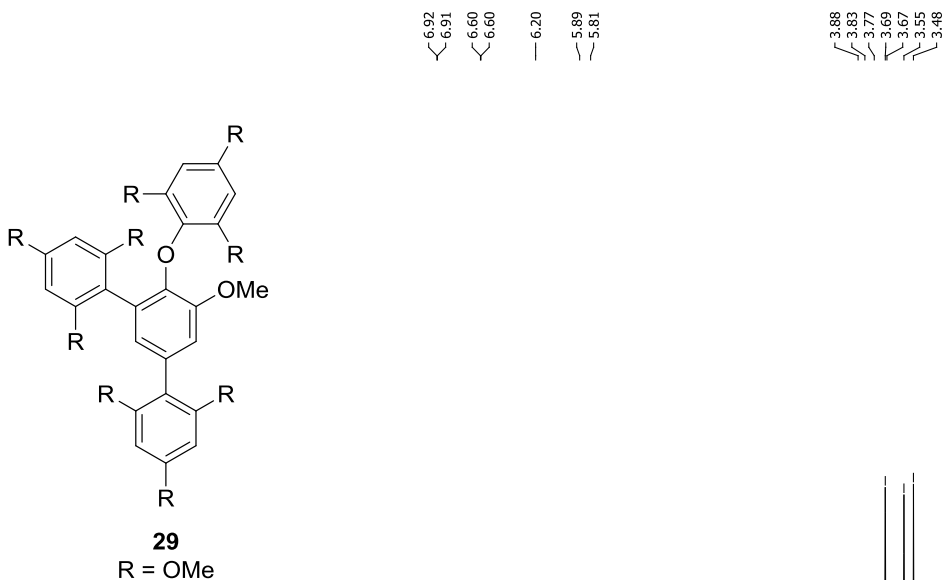

$\mathrm{R}=\mathrm{OMe}$

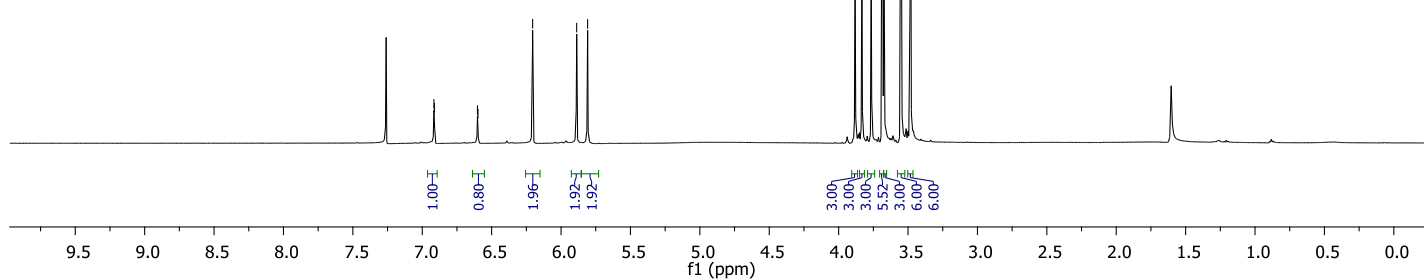

${ }^{13} \mathrm{C}$ NMR spectrum of compound $29\left(\mathrm{CDCl}_{3}, 100 \mathrm{MHz}\right)$
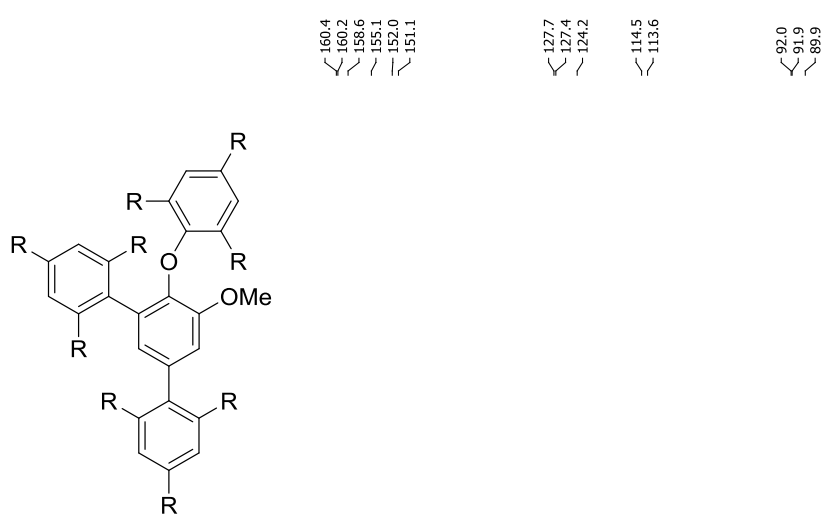

29

$\mathrm{R}=\mathrm{OMe}$

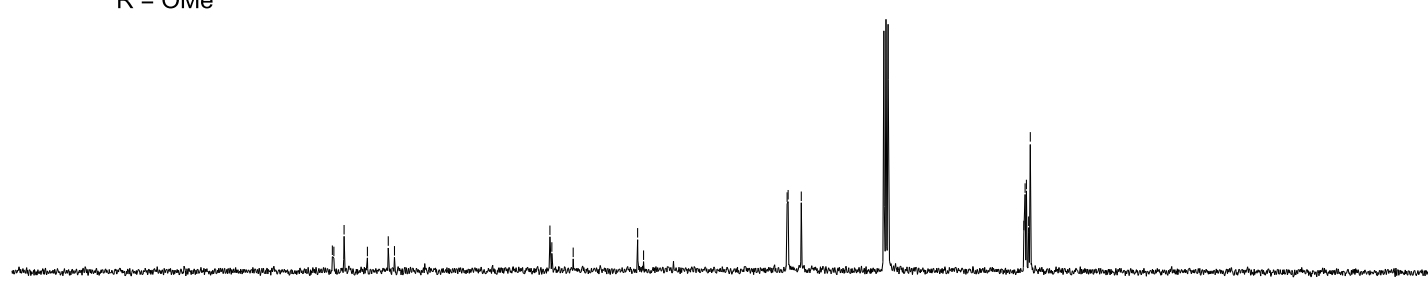

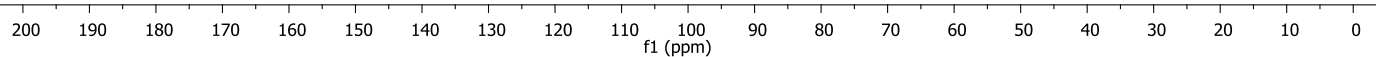

